\title{
A Matched Field Processing Approach \\ to Long Range Acoustic Navigation
}

by

Max Deffenbaugh

B.S.E.. Electrical Engineering

Princeton University (1991)

Submitted in partial fulfillment of the requirements for the degree of

MASTER OF SCIENCE IN OCEANOGRAPHIC ENGINEERING

at the

MASSACHUSETTS INSTITUTE OF TECHNOLOGY

and the

WOODS HOLE OCEANOGRAPHIC INSTITUTION

February 1994

\begin{tabular}{|c|}
\hline $\begin{array}{l}\text { MARINE } \\
\text { BIOLCGICAL } \\
\text { LABORATORY }\end{array}$ \\
\hline LIBRARY \\
\hline $\begin{array}{l}\text { WOODS HOLE, MASS. } \\
\text { W. H. O.I. }\end{array}$ \\
\hline
\end{tabular}

(c) Max Deffenbaugh, 1994. All rights reserved.

The author hereby grants to M.I.T. and W.H.O.I. permission to reproduce and to distribute copies of this thesis document in whole or in part.

Signature of Author

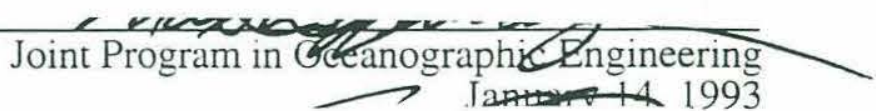

Certified by Prof. Penrik Schmidt

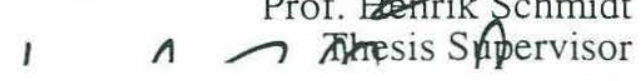

Certified by
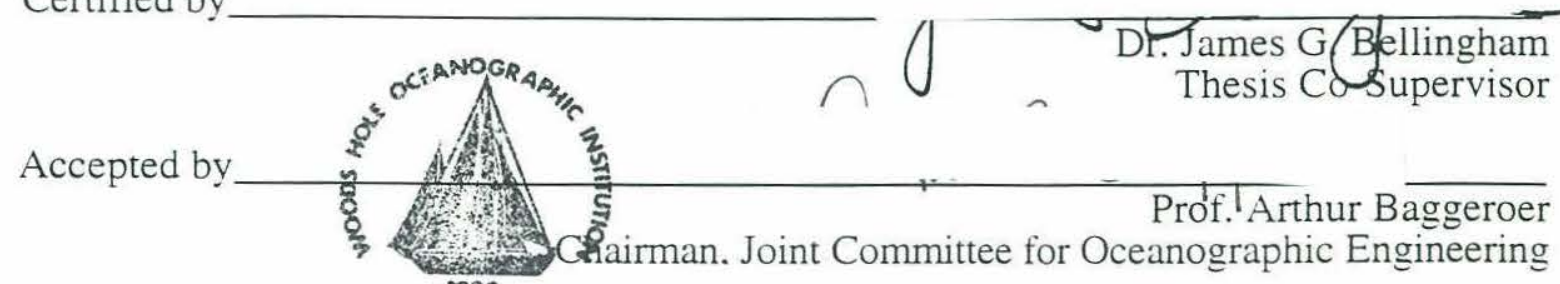

Prof. Arthur Baggeroer

1930 


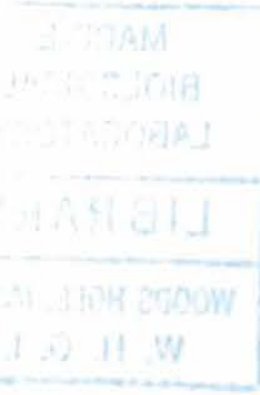




\title{
A Matched Field Processing Approach to Long Range Acoustic Navigation
}

\author{
by \\ Max Deffenbaugh \\ Submitted to the Department of Electrical Engineering \\ on January 14, 1994 in partial fulfillment of the requirements \\ for the degree Master of Science.
}

\begin{abstract}
This thesis presents the design and simulation of an acoustic listen-only navigation system for use in a time-varying high-multipath environment. The system uses a ray tracing model of sound propagation in a horizontally stratified ocean to associate each multipath arrival with a particular ray path through the ocean. This ray path identification allows an inversion for both vehicle position and sound speed profile parameters. Tracking changes in the sound speed profile allows the vehicle to maintain navigation accuracy in a time-varying acoustic environment. Simulation results for typical Arctic conditions indicate the potential for accurate navigation from acoustic beacons at ranges up to tens of kilometers.
\end{abstract}

Thesis Supervisor: Henrik Schmidt

Title: Associate Professor of Ocean Engineering

Thesis Co-Supervisor: James G. Bellingham

Title: Manager, Autonomous Underwater Vehicles Laboratory 



\section{Acknowledgements}

I would like to express my appreciation to my wife for her support and constant love through the ups and downs and late nights at the lab that come with completing an experimental thesis. I would like to thank my father for spending a few weeks bailing me out of a major hardware crunch. Despite the late nights its a memory I will long cherish. I would like to thank Prof. Henrik Schmidt for serving as thesis supervisor and Dr. Jim Bellingham for serving as thesis co-supervisor. I also wish to acknowledge the support received from a National Science Foundation Graduate Fellowship under Grant \#RCD9154652 and from the Office of Naval Research Arctic Programs Office, which made possible this work. 



\section{Table of Contents}

Abstract . . . . . . . . . . . . . . . . . . . 3

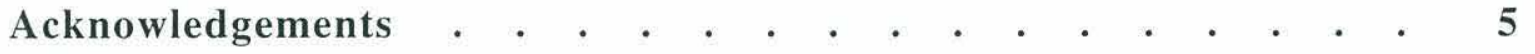

I. Introduction

1.0 Under-Ice Mapping Mission . . . . . . . . . . . . . . . . . 9

1.1 Acoustic Navigation . . . . . . . . . . . . . . . . . . . . 10

1.2 Thesis Overview . . . . . . . . . . . . . . . . . . . . 22

II. Multipath Navigation

2.0 Introduction . . . . . . . . . . . . . . . 25

2.1 Approximation . . . . . . . . . . . . . . 27

2.2 Choice of Measurable Quantities . . . . . . . . . . . . 29

2.3 Choice of Parameters . . . . . . . . . . . . . . . . . . . . . 31

2.5 Parameter Uncertainty Bounds . . . . . . . . . . . . 52

2.4 Signature-Based Navigation . . . . . . . . . . . . . . . . 56

2.6 Linear Approximation ․ . . . . . . . . . . . . . . . . . 58

2.7 Revised System Structure $\quad . \quad$. . . . . . . . . . . . . . . 65

III. Arrival Matching

3.0 Introduction . . . . . . . . . . . . . . . . . 67

3.1 Least Distance Matching . . . . . . . . . . . . . . . . . . $\quad .68$

3.2 Optimal Matching . . . . . . . . . . . . . . . . . . . . . 70

3.3 A Fast Matching Algorithm ․ . . . . . . . . . . . . . . 74

3.4 Importance of Correlations for Matching . . . . . . . . 77

IV. Parameter Estimation

4.0 Introduction . . . . . . . . . . . . . . . . . 81

4.1 Linear Estimates . . . . . . . . . . . . . . . . 82

4.2 Position Estimation Error . . . . . . . . . . . . . . . . 84

4.3 Time Syncronization Estimation Error ．．．．．． . . . 85

4.4 Estimation of the EOF Weights . . . . . . . . . . . 86

4.5 Summary . . . . . . . . . . . . . . . . . . . . . . 88 
V. Performance Evaluation

5.0 Introduction . . . . . . . . . . . . . . . . . . . . . 89

5.1 Sample Mission . . . . . . . . . . . . . . . . . . . 90

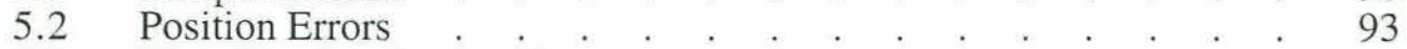

5.3 Time Synchronization Errors . . . . . . . . . . . . . . . . . 96

5.4 EOF Weight Errors . . . . . . . . . . . . . . . . . 98

VI. Conclusion . . . . . . . . . . . . . . . . . 101

Appendix

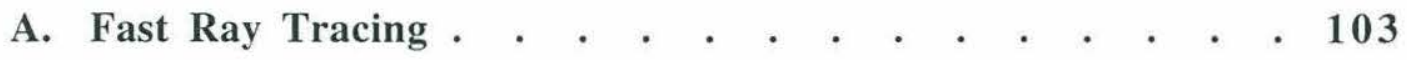

B. Derivative Calculations . . . . . . . . . . . . 105

Bibliography . . . . . . . . . . . . . . . . . . 117 


\section{Chapter 1 Introduction}

\subsection{UNDER-ICE MAPPING MISSION}

In the spring of 1994, the dynamic and mechanical behavior of the Arctic ice cover will be studied from an ice camp deployed in the Beaufort Sea, 2-300 nm north of Prudhoe Bay. The periodic measurement of the under-ice topography is a key component of the experiment, and here the use of autonomous underwater vehicles (AUVs) provides a costeffective alternative to the use of nuclear submarines. A particular challenge in the use of underwater vehicles for under-ice survey is navigation over the large area of experimental interest, perhaps $300 \mathrm{~km}^{2}$ [Bellingham et al., 1993; Deffenbaugh et al., 1993].

The underwater vehicle is shielded from radio navigation systems like GPS or Loran [Sonnenberg, 1988] because of the conductivity of sea water, so position determination must be accomplished acoustically [Milne, 1983]. However, under-ice acoustic navigation over a $300 \mathrm{~km}^{2}$ area is difficult. To keep the number of navigation beacons manageable, the vehicle must be able to utilize beacons as far as $10 \mathrm{~km}$ away. The Arctic acoustic environment at the shallow vehicle depths required for accurate ice mapping is such that significant refraction and time-varying multipath will be present at these ranges.

In this chapter, current practice in underwater acoustic positioning is reviewed. The dual problems of refraction and multipath make current methods ineffective at long ranges. Techniques for dealing with refraction are described, and then techniques for dealing with multipath are discussed. Finally, an overview of the remainder of the thesis is given. 


\subsection{ACOUSTIC NAVIGATION}

There are two common types of acoustic navigation systems. These are distinguished by the quantities they measure. The first type measures range and bearing to a single transponder at a known location. In this category are ultra-short baseline (USBL) systems. In an ultra-short baseline system, the vehicle sends out an acoustic signal. When the transponder hears this signal, it replies with a different signal. The transponder reply is then monitored by a small acoustic array on the vehicle. Range to the transponder is determined from the two-way acoustic travel time. Bearing to the transponder is determined from the small time differences between when the various array elements on the vehicle receive the transponder signal. Such systems work well for homing applications but are less appropriate for a mapping mission like the one described in Section 1.0 because as range from the beacon increases, a constant size bearing error translates into an increasingly large position error. A mapping mission requires a navigation system capable of providing accurate position throughout a large area.

The second type of acoustic navigation system determines position by measuring ranges (only) from three or more acoustic transponders at known locations. Such systems are known as long baseline (LBL) navigation systems. In a long baseline system, the vehicle sends out an acoustic signal. When the various transponders hear the vehicle signal, they each reply at their own unique frequency. When the vehicle receives a transponder response at a particular frequency, it can determine its range to the transponder of that frequency from the two-way acoustic travel time. Once the vehicle has received all the transponder responses, it determines its range from each transponder and triangulates its position accordingly. Such systems provide good accuracy throughout the whole area inside the transponder network and are therefore best suited to mapping missions. 


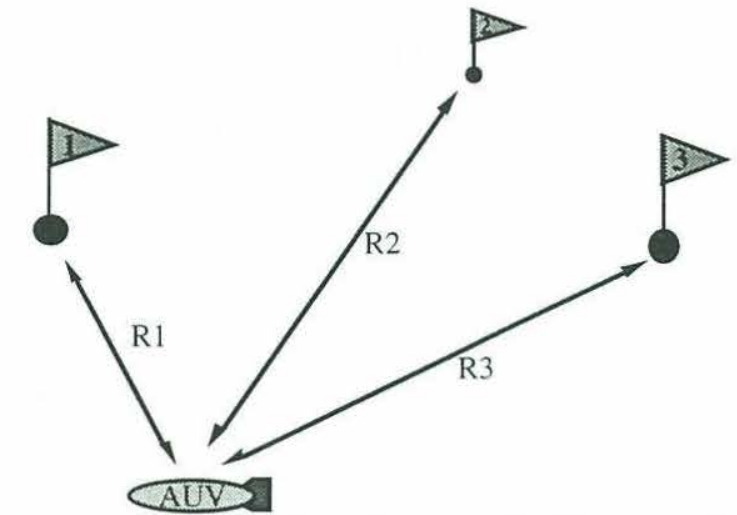

Figure 1.1: Long Baseline Navigation. The vehicle position is triangulated based on range measurements from three or more acoustic transponders.

In the long baseline navigation system described above, the two-way acoustic travel time from the vehicle to the transponder and back gives a range to the known transponder location. This range to a known location defines a sphere centered at the transponder on which the vehicle must lie, since a sphere is the set of all points equally distant from a center point. Responses from several transponders, then, define several spheres, the intersection of which is the vehicle location. This type of long baseline navigation is called "spherical" navigation, since the vehicle position is determined by an intersection of spheres.

Navigation from transponders is difficult if multiple vehicles are to operate within a single transponder network since transponder responses could be confused. The transponders may be replaced with acoustic beacons which transmit at fixed times without vehicle interrogation. Navigation in such a network requires only that the beacons be synchronized in some way so that they all transmit at the same time or with known delays relative to each other. In this scenario, the time differences between when the various beacon signals are received yield differences in ranges to the beacons. The set of points which differ in distance from two foci by a constant amount is a hyperboloid in space. If time differences for three beacon pairs (requiring four beacons) can be measured, then the 
vehicle position can be determined as the intersection of three hyperboloids. This method is known as "hyperbolic" navigation, since the vehicle location is determined by the intersection of hyperboloids. In underwater navigation, depth can be measured simply using a pressure transducer, and the addition of a depth constraint reduces the number of beacons required by one. In such a three-beacon system, care must be taken in the choice of beacon location. There are regions where the hyperboloids of position will intersect in two places, yielding two possible solutions for vehicle location. Usually, these solutions will not cause confusion because their separation will be greater than the uncertainty in vehicle position. In some regions, in particular near the baseline extensions, the two solutions may be close together and direction of travel information will have to be used to identify the correct vehicle path across the ambiguous regions [Bellingham, 1992; Sonnenberg, 1988].

Synchronization between beacons is possible by several methods. The beacons could each have a highly stable time source with pre-programmed transmission times, as done by the GPS system, or the beacons could trigger each other in some sequence as done by the Loran system, or finally, they could all be synchronized to some common time source, such as GPS time or WWVB [Horowitz and Hill, 1989; Sonnenburg, 1988].

The long baseline navigation system must have the ability to convert acoustic travel time to range. Over short distances, this is simple. The acoustic travel time from beacon to vehicle is multiplied by the speed of sound to find the range.

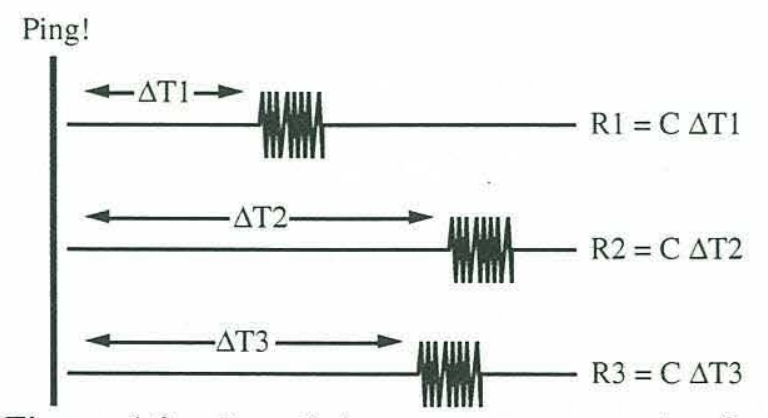

Figure 1.2: Travel time-to-range conversion for the system in Figure 1.1. Range is proportional to acoustic travel time 
Over ranges greater than about one kilometer, however, sound velocity variations in the ocean complicate the relationship between travel time and range, and a more sophisticated model of the acoustic environment is necessary.

\section{CORRECTING FOR REFRACTION}

In the ocean, temperature, salinity, and pressure variations with depth create a depth-dependent sound speed profile [Frisk, 1994]. In general, the horizontal variability of sound speed is much smaller and may be ignored for the horizontal ranges of interest.

As a sound ray passes through this speed-varying medium, it is subject to refraction. In the case where all ray paths are nearly straight, a useful approximation can be made to simplify the range-time relationship. Fermat's principle observes that the path a ray follows is a (local) extremum of travel time over all paths between source and receiver [Frisk, 1994]. Thus for differential perturbations in ray path, the travel time does not change. By the same principle, if a small perturbation is applied to the sound speed, the actual ray path is perturbed slightly, but the travel time may be calculated as if the ray path had remained the same through the new medium. This leads to the method of calculating a depth-dependent effective sound speed as the inverse of the average sound slowness between two depths [Van Calcar, 1985].

$$
\frac{1}{c_{e f f}\left(z_{1}, z_{2}\right)}=\frac{1}{z_{2}-z_{1}} \int_{z_{1}}^{z_{2}} \frac{d z}{c(z)}
$$

This method is based on the assumption that the ray path is close to straight so the travel time can be calculated as if it were indeed straight. In this approximation, the effective sound speed is independent of the ray angle since for straight paths the proportional influence of slowness at each depth is the same. 
The limitations of this straight path approximation can be seen by using Snell's Law to find the rate of change in ray angle with respect to horizontal distance. Here, $\theta(z)$ is the ray angle as a function of depth, $z$. The sound speed profile is $c(z)$, and $a$ is the ray parameter, $\cos \theta(z) / c(z)$, which Snell's law states is constant over depth [Frisk, 1994].

$$
\begin{gathered}
\frac{\cos \theta(z)}{c(z)}=\mathrm{a} \\
\frac{d \theta(z)}{d x}=\frac{d}{d x} \cos ^{-1}(a c(z)) \\
=-\frac{a}{\sqrt{1-(a c(z))^{2}}} \frac{d c(z)}{d z} \frac{d z}{d x}
\end{gathered}
$$

The straight path approximation will fail, then, when the ray path is sufficiently long to accumulate a significant change in angle. Greater angle changes will be accumulated when the path is close to horizontal, that is when $a c(z)$ is close to one, and when the sound speed gradient, $d c(z) / d z$, is large.

The Arctic mission requires navigation over a $300 \mathrm{~km}^{2}$ area. To do this with a reasonable number of beacons requires that the vehicle be able to use beacons at ranges of up to $10 \mathrm{~km}$. The travel time inaccuracy produced by the straight path approximation is plotted below as a function of vehicle range and depth for a typical Arctic sound speed profile. The beacon is at zero depth and zero range. The typical Arctic sound speed profile in Figure 1.3 is the average of ten profiles taken over a five-day period in the Greenland Sea [Scheer]. 


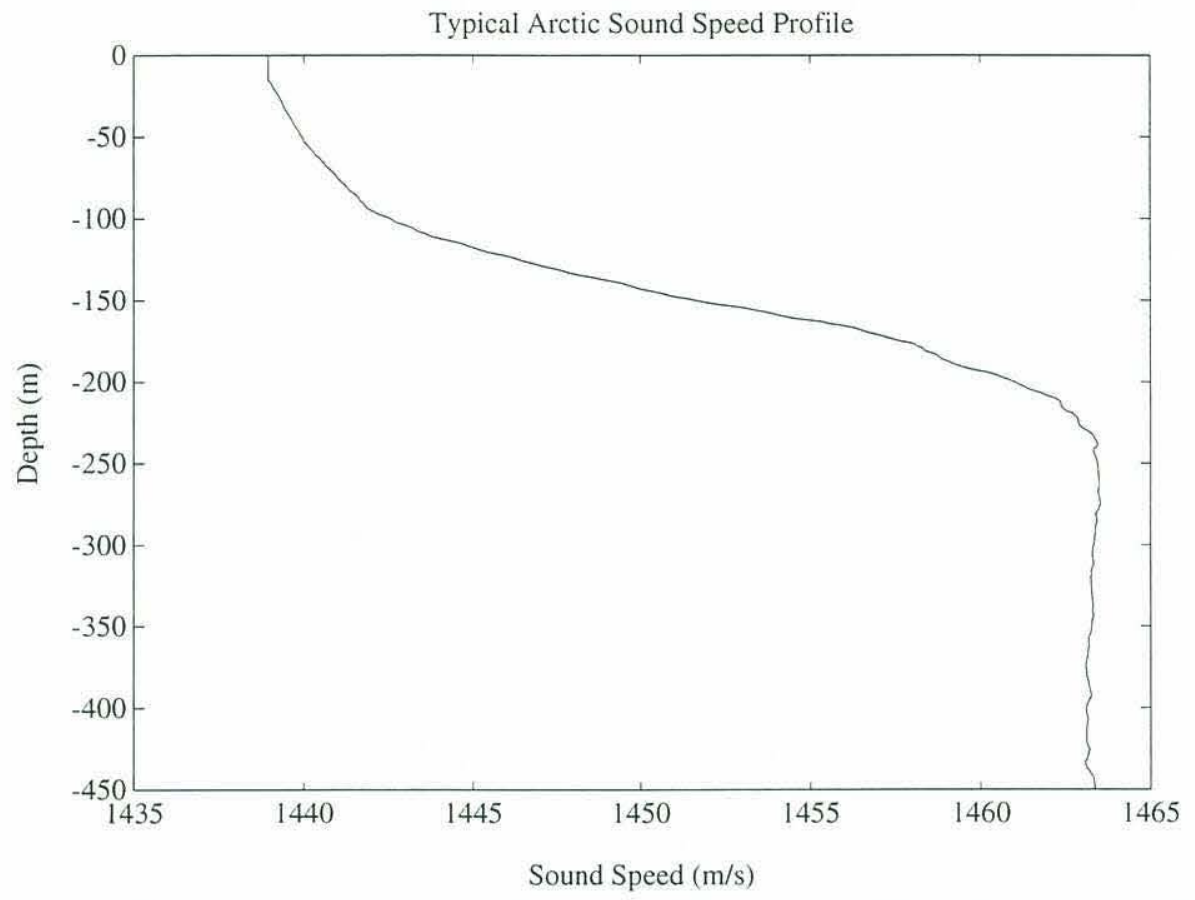

Figure 1.3: Typical Arctic sound speed profile. This profile is the average of a series of ten casts taken over a five day period.

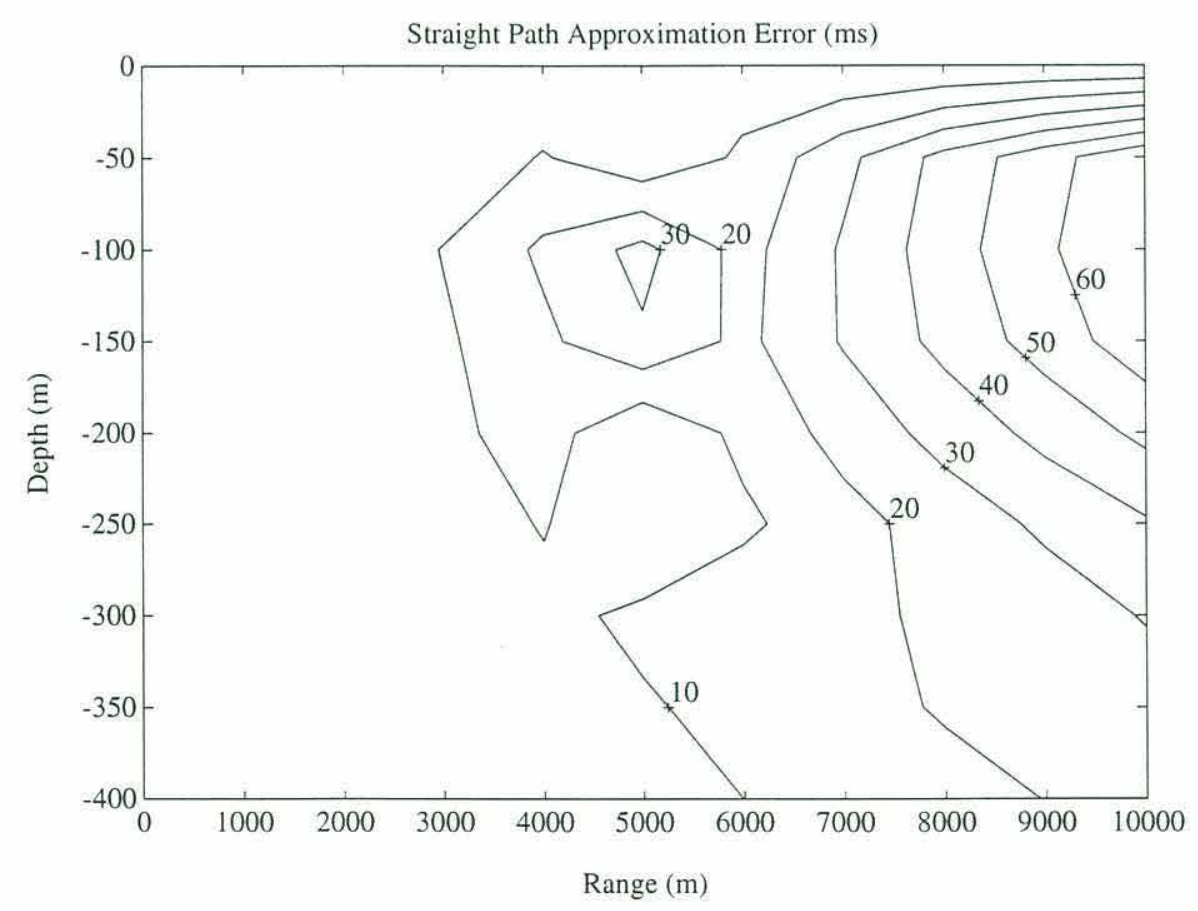

Figure 1.4: Travel time error resulting from the straight path approximation. This graph shows the approximation error from using the straight path approximation to predict the travel time for the first acoustic arrival. The beacon is at zero depth and zero range, and the approximation error over realistic vehicle depths and horizontal ranges is shown. 
It was demonstrated that the straight path approximation is most useful for rays at steep angle, modest sound speed gradients, and short ranges. Accordingly, the straight path approximation error is smaller at great vehicle depth, is smaller very close to the surface where the sound speed gradient is small, and is smaller at short ranges. In the regime of shallow depths and long ranges required for the ice mapping mission, the straight-ray approximation is inadequate. A full ray tracing model will be required to predict acoustic travel times.

\section{MULTIPATH NAVIGATION}

The characteristic acoustical feature of the Arctic Ocean is a shallow upwardrefractive sound channel. At shallow depth, this sound channel creates significant multipath in the form of refracted-surface-reflected rays at ranges beyond a kilometer. The figure below shows the non-bottom interacting eigenrays which connect a source and receiver at $30 \mathrm{~m}$ depth at a range of 10 kilometers for the sound speed profile of Figure 1.3.

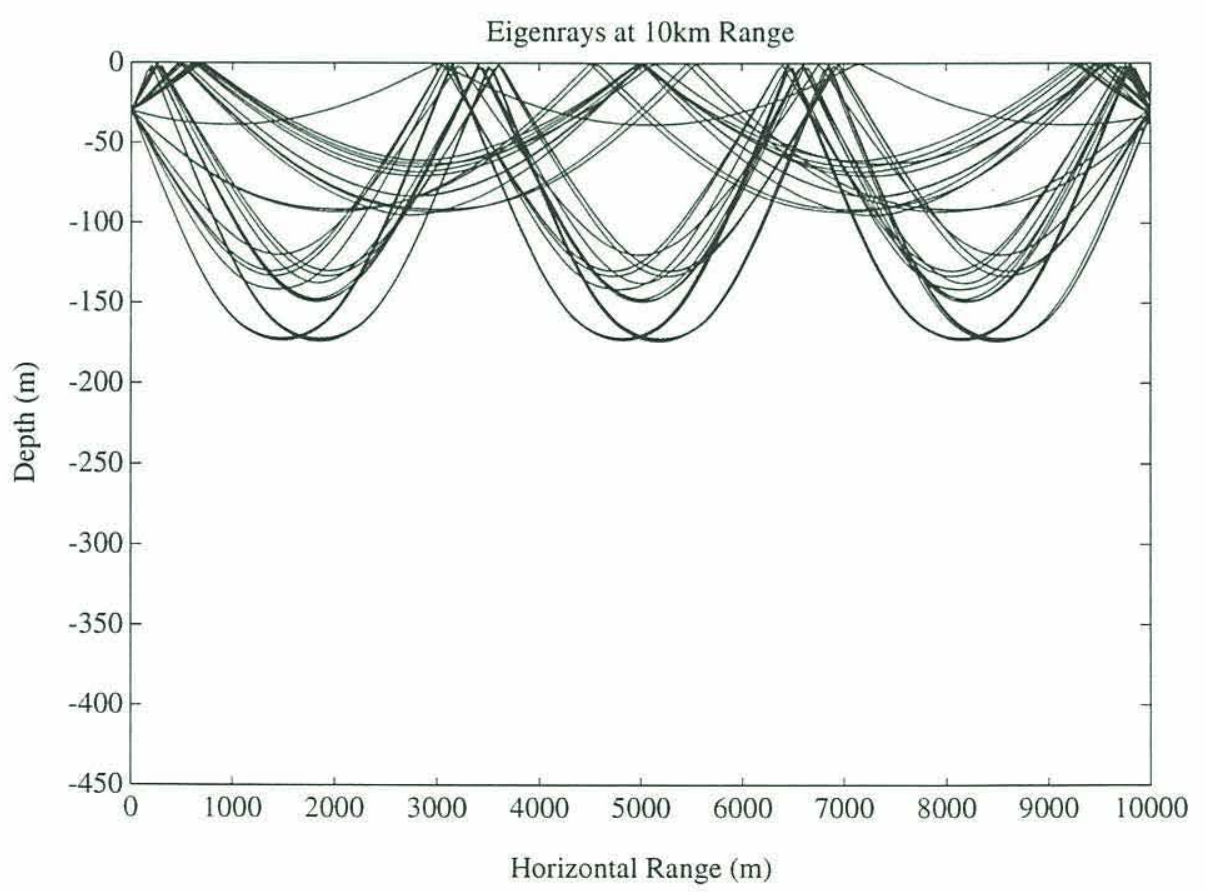

Figure 1.5: Eigenrays for source and receiver at $30 \mathrm{~m}$ depth and $10 \mathrm{~km}$ range. This ray tracing is based on the sound speed profile of Figure 1.3. 
Each of the eigenrays in Figure 1.5 has a characteristic acoustic travel time. For each signal the beacon transmits, multiple arrivals of the signal will be observed at the receiver. The time of each arrival depends on the vehicle position and therefore contains information about where the vehicle is located. The challenge in multipath navigation is to extract this position information from the multipath signal.

Two classes of techniques have been applied to this problem. The distinguishing characteristic of the two classes is whether the received acoustic signal is viewed as a single entity--an acoustic "signature" corresponding to a particular location, or as a collection of entities--a set of arrivals from different identifiable ray paths.

In the first class of techniques, which might be called "signature-based" approaches, the received signal is viewed as a single entity--a "signature" characteristic to a particular location. The set of possible positions is searched by some method, such as simulated annealing, until the position is found for which the acoustic signature predicted by the model most closely matches the measured one. Using this technique, the potential for three-dimensional positioning with a single beacon and a single hydrophone while within the high-multipath environment of an oil rig structure has been demonstrated [Coates and Wang, 1993]. The multipath arrival times for each position within the oil rig structure are pre-computed, and the position for which the predicted set of arrivals best matches the measured arrivals is taken to be the true position. The disadvantage of signature-based approaches is that the search for the optimal position may be time consuming, particularly if a sophistical sound propagation model must be employed to determine the predicted acoustical signal for each possible position.

The second class of navigation techniques, which might be referred to as ray-based approaches, attempts to identify each multipath arrival in the received signal with a particular ray path through the water. Once this identification is made, the behavior of each ray path can be understood separately in terms of how its travel time is effected by changes 
in position. Thus, the travel times for each identified ray path can be used to directly invert for vehicle position. A classical example of this technique is "single-hydrophone" ranging [Burdic, 1991]. In a straight ray path shallow water environment, surface and bottom reflected paths can be used in conjunction with the direct path to yield both range and depth of a distant acoustical source. The relative travel times along the identified ray paths can be directly related to source position, so a search over numerous possible positions is unnecessary. Ray-based approaches find the true vehicle position faster than signaturebased approaches by utilizing the physics of the rays which compose the received signal; however, their success depends on the ability to identify acoustic arrivals in the multipath structure with particular ray paths.

\section{- The Fading Problem}

The ability to use a particular acoustic arrival for ray-based navigation depends on the ability to determine which ray path it followed through the water. Once the ray path of an arrival has been determined, the relationship between small changes in vehicle position and small changes in travel time for that ray path can be linearized around an estimate of vehicle position. The difference between the measured travel time and the predicted travel time for each ray can be used to invert for an improved vehicle position estimate. The difficulty in Arctic multipath navigation is that small changes in the sound speed profile over time can cause individual ray paths to fade, and ice keels, which may extend to depths of $30 \mathrm{~m}$, may block certain ray paths near their surface reflection points. If fading of one ray path causes a misidentification of another arrival such that the position-time linearization for the faded ray path is accidentally applied to the wrong arrival, an error will occur in the updated position estimate. Suppose, for example, that the vehicle expects to hear arrivals at times $t_{1}$ and $t_{2}, t_{1}<t_{2}$, from ray paths $p_{1}$ and $p_{2}$. Unexpectedly, a small change in the

sound speed profile has occurred such that path $p_{1}$ no longer exists. The first arrival the vehicle hears is at time $t_{2}$, along path $p_{2}$. However, the vehicle assumes that the first 
arrival will come along path $p_{1}$, and so it adjust its position estimate in such a way as to cause the predicted travel time for path $p_{1}$ to equal $t_{2}$. This creates a substantial error in the position estimate.

Path fading also presents a difficulty for signature recognition systems. When a certain ray path disappears, the predicted signature for a particular location no longer matches the measured signature at that location. This could create an error in the position estimate.

\section{- Navigation in Fading Environments}

Path fading presents a particular challenge to navigation systems because it is difficult to predict. Fortunately, however, fading is unusual in the sense that in realistic environments most of the ray paths are present at any given time. For these two reasons, systems which deal with fading tend to do so by creating some sort of redundancy to enable isolation of fading events.

One ray-based method for dealing with the arrival fading problem in a system which uses only first arrivals is to assume that the earliest detected arrival from each beacon is the earliest predicted arrival. A beacon range error will result if the earliest detected arrival is not, in fact, the earliest predicted arrival. However, if ranges are measured from enough acoustic beacons so that the vehicle position calculation is over-determined, the ranges based on correctly identified arrivals will produce a consistent vehicle position estimate, while any ranges based on misidentified arrivals will be found to be inconsistent. Thus based on a consistency criterion, the erroneous ranges may be detected and purged [Duckworth, 1987].

A second sort of redundancy can be achieved by observing the multipath over time. A first arrival recording system can actually use fading to advantage if its motion during the time between path fading events is small. The occasional fading of arrivals means that a system which logs the travel time for the first arrival it hears will end up logging travel 
times for several different ray paths. This allows a simple first-arrival receiver to acquire several multipath delays for its position if it stays in the same place long enough. This fact has been exploited for position estimation of array elements suspended from ice floes. Once a set of several multipath delays is acquired, a simulated annealing algorithm is used to find the position most consistent with the observed multipath structure [Von der Heydt and Scheer].

The simplest receiver system to build is one which records only the first arrival, as do both of the above systems. In a multipath signal, however, all the arrivals contain useful information about the vehicle position. If all arrivals could be recorded, the redundancy necessary to identify faded arrivals could be had within the received signal from a single beacon, rather than requiring the use of additional beacons, as in the first system, or extended observation times, as in the second system.

\section{- Sound Speed Profile Estimation Using Multipath}

The ray tracing which enables the association of travel times with vehicle position depends on accurate knowledge of the sound speed profile. If changes in the sound speed profile over time render the ray tracing inaccurate, biases will be introduced in the position estimate. If profile changes could be estimated, then the navigation system could maintain accuracy indefinitely. The problem of using acoustic travel times in a multipath environment to estimate both acoustic source location and the sound speed profile has been addressed in the context of ocean acoustic tomography [Cornuelle, et al., 1989]. Tomographic techniques form an incomplete answer to the Arctic navigation problem, however, because the multipath in the shallow Arctic sound channel is less stable and much more susceptible to fading than in the deep ocean. Travel time variability is also much greater, since position uncertainty has a larger effect, making path identification the central challenge in the Arctic. 
A method of location finding in an uncertain environment has appeared in the source localization literature [Collins and Kuperman, 1991]. In this approach, the detected eigenrays are back-propagated, and sound speed profile parameters are perturbed via simulated annealing until the eigenrays all focus on a single location. This focal point is taken to be the source location. This method has the attractive feature of being completely robust to path fading, since only the detected arrivals are used. It also allows appearance of an unexpected path--one that the current profile estimate indicates should be faded--as long as the path is predicted by the correct choice of sound speed profile parameters. A problem arises, however, if an unexpected path appears which cannot be predicted at the correct location by any choice of parameters. In the Arctic sound channel, this could be caused by reflection off of a non-level part of the ice or some unmodeled feature in the sound speed profile, such as transient fine structure. In such a case, the algorithm may fail to focus at the correct location. A consistency test based on the redundancy of having many rays which must focus at a single location could be used to purge an occasional misbehaving ray. Unfortunately, there are two practical problems with implementing such a method on an AUV. First, the method requires extremely accurate measurement of ray arrival angles for effective back-propagation, and it would be difficult to mount an array of sufficient length on an AUV, even at the high frequencies used for navigation. Second, the computational demands of this approach render it impractical for real-time operation in AUVs with limited space and power budgets. 


\subsection{THESIS PREVIEW}

This thesis presents a navigation system which uses all the detectable arrivals of each beacon signal to invert for both vehicle position and sound speed profile in a fading multipath environment. The system takes a matched field approach to the navigation

problem. Based on an initial estimate of the position and sound speed profile, a predicted received signal is generated by a propagation model. This predicted signal is compared with the signal the vehicle actually measures, yielding some prediction error. This prediction error is then inverted to update the estimate of position and sound speed profile.

Central to the system, then, is a sound propagation model which allows changes in position and sound speed profile to be related to changes in the received signal. Chapter 2 discusses this model. The approximations in the model are presented, and the measurable quantities--arrival times and arrival angles--are identified and related to the unknown parameters--vehicle $\mathrm{x}$ - and $\mathrm{y}$ - coordinates, vehicle clock error, and three weights for the three basis functions used to approximate the sound speed profile. It is shown that there is a linear relationship between the measurable quantities and the unknown parameters over typical regions of parameter uncertainty. This motivates the division of the navigation problem into an arrival matcher, which seeks to associate each measured arrival with a particular predicted ray path, and a linear inversion, which uses the identified arrivals to invert for an updated parameter estimate.

Chapter 3 examines the problem of arrival matching. Two methods are discussed. The first is a least-distance algorithm. This algorithm does not take into account correlations in the shifts between the acoustic travel times for the various arrivals, so a criterion for correlated matching is proposed, and a fast matching algorithm which implements this criterion is presented. Finally, the performance of the two matching methods is compared. 
Chapter 4 examines the linear inversion portion of the system in greater detail. This section operates on the assumption that all the arrivals have been correctly matched, and based on this assumption shows error variances for position, time synchronization, and each of the sound velocity profile parameters.

Chapter 5 presents the results of a simulated mission. The vehicle is given a course to navigate, and the navigation accuracies of a standard hyperbolic navigation system, a first-arrival matching system, and the multipath-matching system are compared. It is observed that it is difficult to resolve the sound speed profile parameters . Reasons for this are discussed.

Chapter 6 contains concluding remarks. The important contributions of this work are identified, and future research directions are outlined.

Appendix A contains a description of the fast ray tracing algorithm used for all the simulations and ray tracing plots in this thesis. Appendix B contains the calculations of the derivatives of the measurable quantities with respect to the unknown parameters. 



\section{Chapter 2 Multipath Navigation}

\subsection{INTRODUCTION}

Central to a multipath navigation system is a sound propagation model which allows the received signal to be predicted for a given position and sound speed profile estimate. The approximations which are made in this model are discussed in Section 2.1. Section 2.2 justifies the choice ray travel times and arrival angles as the measurable features of the received signal, to the exclusion of amplitude and phase information which is difficult to predict accurately. Section 2.3 presents the system parameters. The vehicle $\mathrm{x}-$ and y-position will be estimated. The beacons are assumed to all be synchronized to GPS time, but the time synchronization error between the vehicle clock and beacon time must be estimated. It is shown that hyperbolic and spherical navigation correspond to the opposite extremes of infinite and zero variance in the knowledge of this time synchronization error. The sound speed profile is represented using the method of empirical orthogonal functions (EOFs), and the first three such functions are shown. Section 2.4 provides bounds on the

uncertainty of the various system parameters. Section 2.5 considers the application of signature-based navigation to such a system. An energy function showing "goodness of match" between predicted and measured travel times and angles for a typical region of uncertainty in beacon range and one sound speed profile parameter is shown. This function is seen to have numerous local maxima, making it a difficult optimization problem. The observation is made that each local maximum corresponds to one or more measured and predicted arrivals lining up for a particular parameter choice. Thus all the peaks of the 
energy function could be found by finding all possible matches between measured and predicted arrivals. Section 2.6 demonstrates that the relationship between changes in travel time and changes in the various parameters is linear over typical regions of parameter uncertainty, as long as the associated ray path does not fade. This observation motivates the division of the position estimation problem into two parts in Section 2.7. The first part is an arrival matching process, where it is attempted to associate each of the predicted arrivals with one of the measured arrivals. This amounts to finding a set of arrivals which do not fade over the region of parameter estimate error. This process can also be interpreted in light of Section 2.5 as finding the peak of the "goodness of match" energy function which has the highest value. The second part of the position estimation problem is, once the best match is found, to perform a linear inversion for the parameter values based on the matched arrivals. The arrival matching process is discussed further in Chapter 3 , and the linear inversion is discussed in Chapter 4 . 


\subsection{APPROXIMATIONS}

At the heart of the matched field navigation system is a sound propagation model which can relate a parameter estimate to the measurable signal. This model will make certain assumptions about the environment. These are spelled out explicitly here.

\section{- Approximation 1: Stratified Ocean}

The horizontal variation in sound speed is generally much smaller than the vertical variation. It will be assumed that the ocean is stratified so that sound velocity is a function only of depth.

\section{- Approximation 2: No Currents}

It is assumed that there are no currents. Inversion for a current profile may be possible since the directionality of current-generated sound speed change would enable it to be separated from temperature- and salinity- generated sound speed change. For now, however, currents will be ignored.

\section{- Approximation 3: Flat, Perfectly Reflective Surface and Bottom}

It is assumed that the surface and bottom are flat and perfectly reflective. In reality there are two sorts of surface roughness. The underside of the ice has an r.m.s. roughness of approximately $2 \mathrm{~m}$ with a correlation distance of $40 \mathrm{~m}$ [Schmidt]. If the predicted travel time is based on the flat surface assumption, it should be possible to translate these roughness statistics into predicted travel time error statistics. In addition to this general roughness profile, large ice keels, as deep as 30m may form at regions of interface between ice floes. For now, however, all surfaces will be flat. 
- Approximation 4: Discrete arrivals

It will be assumed that the arrivals along the various eigenrays can be detected and separated. The beacon transducers currently employed offer an $8 \mathrm{kHz}$ bandwidth, while the arrival time separations tend to be on the order of milliseconds.

\section{- Approximation 5: Depth is Known}

Depth can be measured simply and accurately with a pressure transducer. It will be assumed that the depths of the vehicle and beacons are known perfectly. 


\subsection{CHOICE OF MEASURABLE QUANTITIES}

At the frequencies which are typically used for acoustic navigation, greater than $8 \mathrm{kHz}$, the ray approximation provides an accurate characterization of the acoustic field [Frisk, 1994]. Under this approximation, the received signal can be fully characterized by considering the travel times, arrival angles, amplitudes and phases of its component eigenrays. Subtle changes in the sound speed profile can cause dramatic changes in ray amplitude, so it is unlikely that amplitude can be predicted accurately, particularly in media which are characterized by significant fine structure such as near-surface waters [Pedersen, 1961]. Phase is also difficult to predict, because at navigation frequencies in the shallow Arctic sound channel, propagation is characterized by saturated scattering [Catipovic and Baggeroer, 1990; Flatté, 1979]. This leaves travel times and arrival angles as the measurable characteristics of the received signal which are likely to be predictable by an acoustic propagation model. It has been noted that in the regime where individual arrivals can be resolved, amplitude and phase information add little to the inversion accuracy [Brown, 1984].

The received signal is then described as a set of points in travel time - arrival angle space. Figure 2.1 shows the eigenrays at a $3 \mathrm{~km}$ range for a source and receiver at a $30 \mathrm{~m}$ depth. Figure 2.2 shows the representation of the received signal as a set of points in travel time - arrival angle space. Both of these plots are generated using the sound speed profile of Figure 1.3. Angles are measured in degrees below horizontal. Notice, for example, that the two ray clusters at intermediate angles in Figure 2.1 appear as two clusters of points in Figure 2.2. 


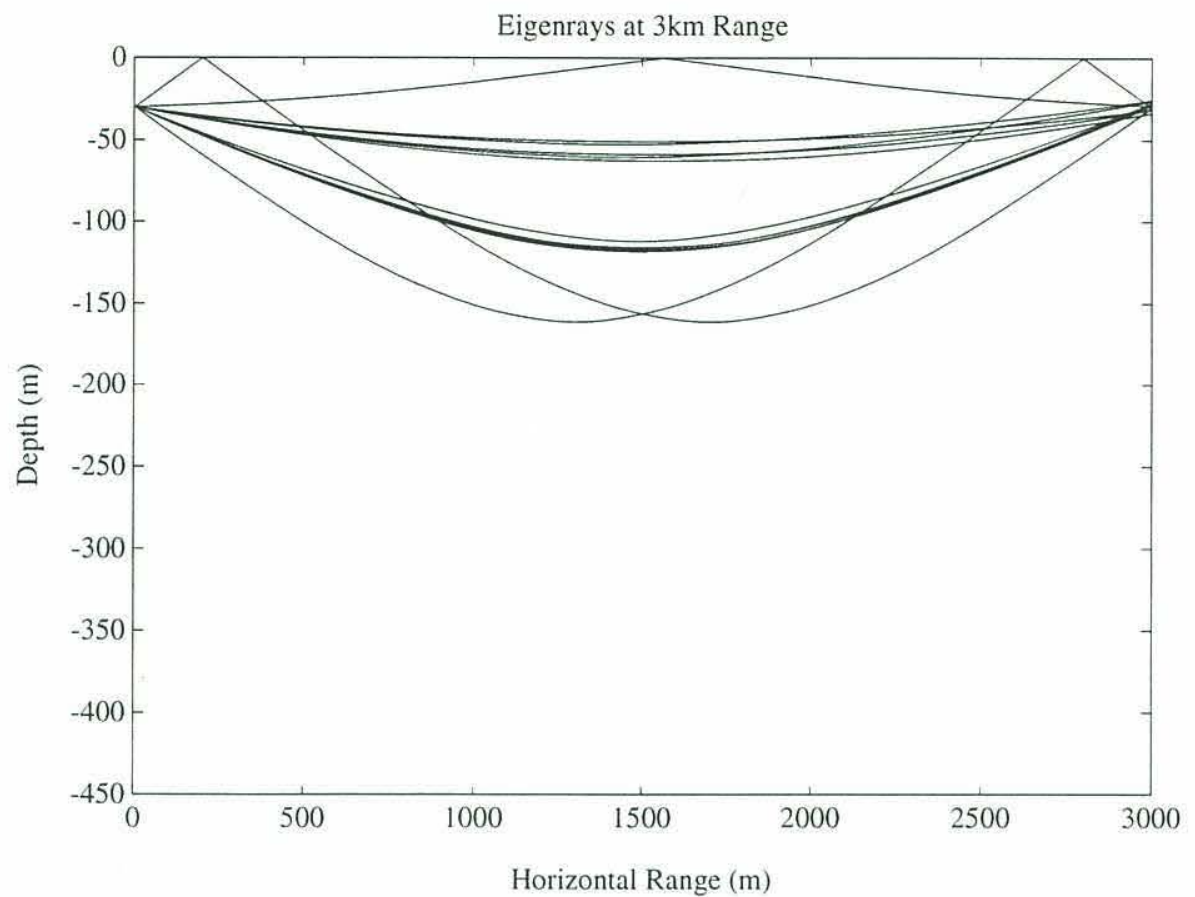

Figure 2.1: Eigenrays at $3 \mathrm{~km}$ range for source and receiver at $30 \mathrm{~m}$ depth. This ray trace is based on the sound speed profile of Figure 1.3.

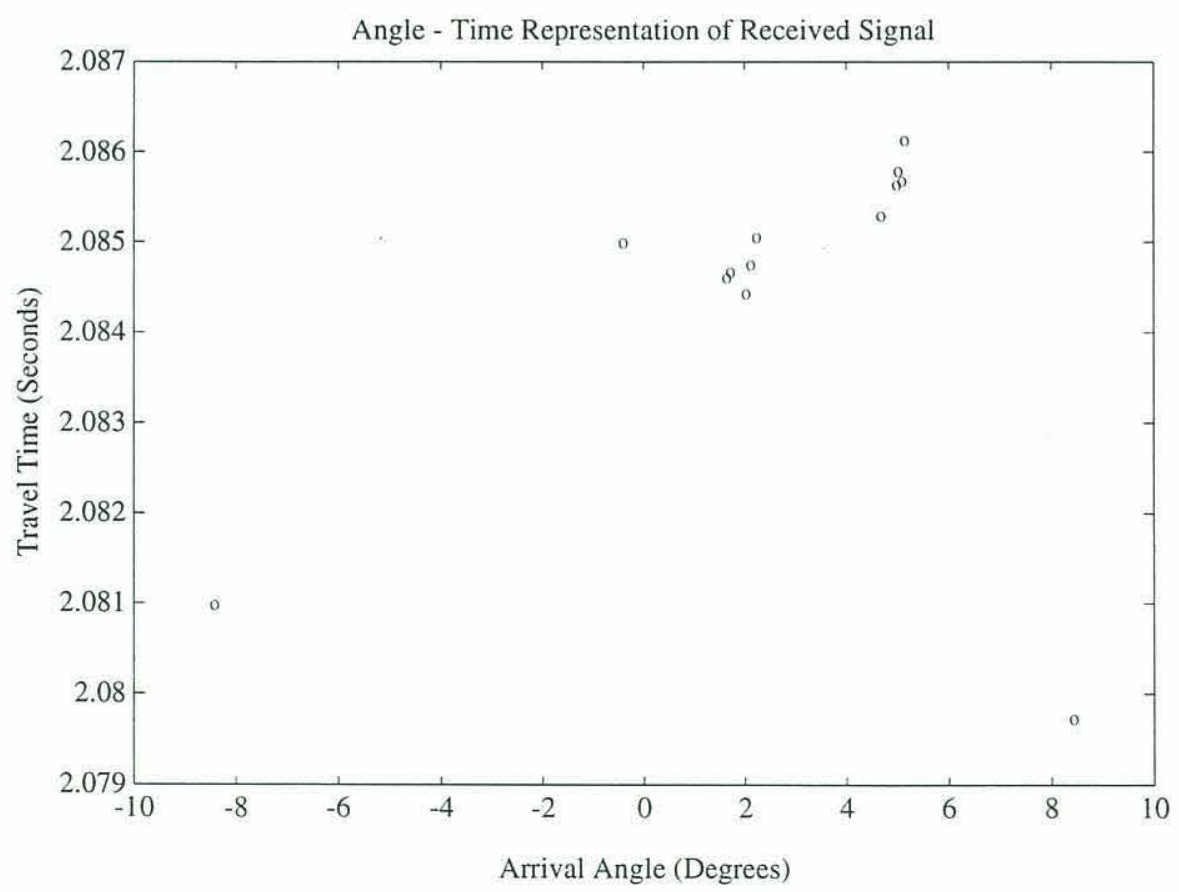

Figure 2.2: Arrival angle-travel time representation of the channel impulse response corresponding to the eigenrays in Figure 2.1. 


\subsection{PARAMETERIZATION}

From a finite number of observations, it can only be hoped to estimate a finite number of parameters. The choice of parameters will effect the ultimate accuracy and robustness of the navigation system.

\section{POSITION MEASUREMENT}

Long baseline navigation systems triangulate vehicle position based on range measurements from beacons or transponders at known locations. The range is calculated from the acoustic travel time to each beacon. If these time measurements are noisy, the accuracy of the estimated range will depend on both the noise level and the vehicle position with within the beacon network. Consider the system,

$$
\mathbf{t}=f(\mathbf{x})+\mathbf{n}
$$

where $\mathbf{t}$ is the vector of measured travel times, $f(\mathbf{x})$ is the true acoustic travel time for a vehicle at location $\mathbf{x}$, and $\mathbf{n}$ is the measurement noise. The Cramér-Rao bound [Nahi, 1969] gives a lower limit on the covariance of any estimator $\hat{\mathbf{x}}=\hat{\mathbf{X}}(\mathbf{t})$ for $\mathbf{x}$.

$$
\begin{gathered}
\mathrm{E}\left[(\hat{\mathbf{x}}-\mathbf{x})(\hat{\mathbf{x}}-\mathbf{x})^{T} \mid \mathbf{x}\right] \geq \mathrm{J}-1 \\
\mathrm{~J}_{i j}=\mathrm{E}\left[\left(\frac{\partial \log p(\mathbf{t} \mid \mathbf{x})}{\partial x_{i}} \frac{\partial \log p(\mathbf{t} \mid \mathbf{x})}{\partial x_{j}}\right)\right]
\end{gathered}
$$

The measurement noise is taken to be Gaussian with covariance matrix R. This yields the probability density function for the travel times, 


$$
\begin{gathered}
p(\mathbf{t} \mid \mathbf{x})=\frac{1}{(2 \pi)^{N / 2}|\mathrm{R}|^{1 / 2}} \exp \left[-\frac{1}{2}\left([\mathbf{t}-f(\mathbf{x})]^{T} \mathrm{R}^{-1}[\mathbf{t}-f(\mathbf{x})]\right)\right] \\
\mathrm{R}=\mathrm{E}\left[\mathbf{n} \mathbf{n}^{\mathrm{T}}\right]
\end{gathered}
$$

The Cramér - Rao bound for this spherical navigation problem is then,

$$
C R B_{S}=\left(\frac{\partial f(\mathbf{x})^{T}}{\partial \mathbf{x}} \mathrm{R}^{\circ}-1 \frac{\partial f(\mathbf{x})}{\partial \mathbf{x}}\right)^{-1}
$$

It is worth noting that the Cramér - Rao bound is realized exactly by the least squares estimate for the case of Gaussian noise. The figure below shows the contours of the bound in a three-beacon network for the standard deviation of position when the standard deviation of measurement noise is $1 \mathrm{~ms}$. Beacons and vehicle are in the same plane. The three beacons are shown as small circles.

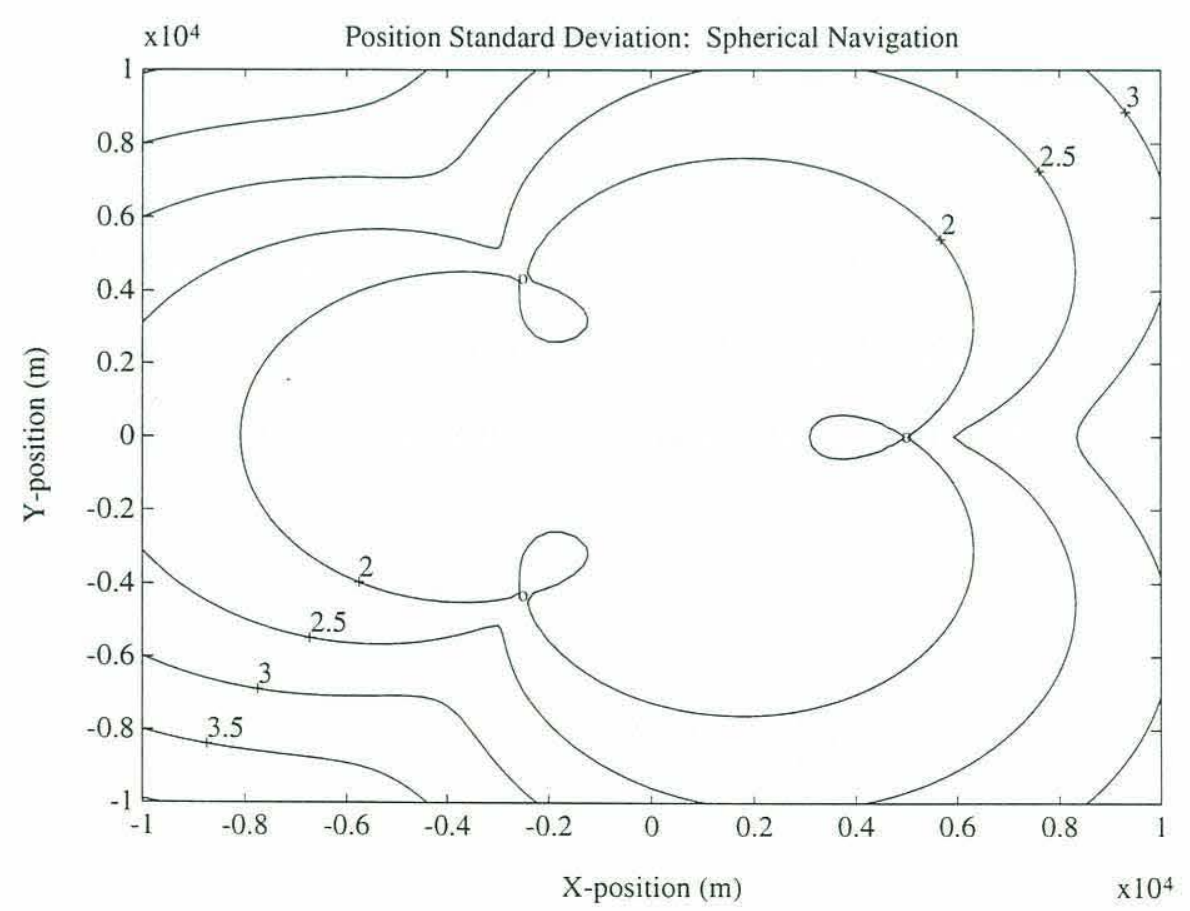

Figure 2.3: Standard deviation of position in meters for spherical navigation with $1 \mathrm{~ms}$ standard deviation in travel time measurement. 
As mentioned in Section 1.1, transponder navigation is difficult if multiple vehicles

are to operate within a single beacon network since transponder responses may be confused. It is desirable for the vehicle to operate in listen-only mode. For the Arctic mission, the navigation beacons will be suspended from the ice. This surface penetration enables them to be synchronized to GPS time so that they transmit simultaneously. However, the vehicle clock will drift with respect to GPS time, so the measured travel times will be corrupted by a common vehicle clock error, $T$.

$$
\mathbf{t}=f(\mathbf{x})+T+\mathbf{n}
$$

As mentioned in Section 1.1, a common approach to dealing with this problematic unknown $T$ is to use time differences between arrivals from beacon pairs, so that the common factor of $T$ cancels out. These time differences define intersecting hyperboloids on which the vehicle must lie. Loran is perhaps the best known radio hyperbolic navigation system [Beck, 1971], and hyperbolic navigation has been applied to underwater vehicles as well [Bellingham, 1992]. The hyperbolic navigation equations can be created by (left) multiplying equation 2.3 .5 by a matrix $\mathrm{M}$, of size $\mathrm{N}-1 \times \mathrm{N}$, where $\mathrm{N}$ is the number of beacons.

$$
\begin{gathered}
\mathbf{M}=\left[\begin{array}{ccccc}
-1 & 11 & 0 & 0 & \ldots \\
-1 & 10 & 1 & 0 & \ldots \\
-1 & 10 & 0 & 1 & \ldots \\
: & 1 & \ldots & \ldots & \ldots
\end{array}\right] \\
\mathbf{M t}=\operatorname{Mf} f(\mathbf{x})+\mathbf{M n}
\end{gathered}
$$

The Cramér-Rao bound for position variance for hyperbolic navigation is then,

$$
C R B_{H}=\left(\frac{\partial f(\mathbf{x})^{T}}{\partial \mathbf{x}} \mathrm{M}^{\mathrm{T}}\left(\mathrm{MRM}^{\mathrm{T}}\right)^{-1} \mathrm{M} \frac{\partial f(\mathbf{x})}{\partial \mathbf{x}}\right)^{-1}
$$


Figure 2.4 shows the contours of the bound in a three-beacon network for the standard deviation of position when the standard deviation of measurement noise is $1 \mathrm{~ms}$. Beacons and vehicle are in the same plane. The three beacons are shown as little circles.

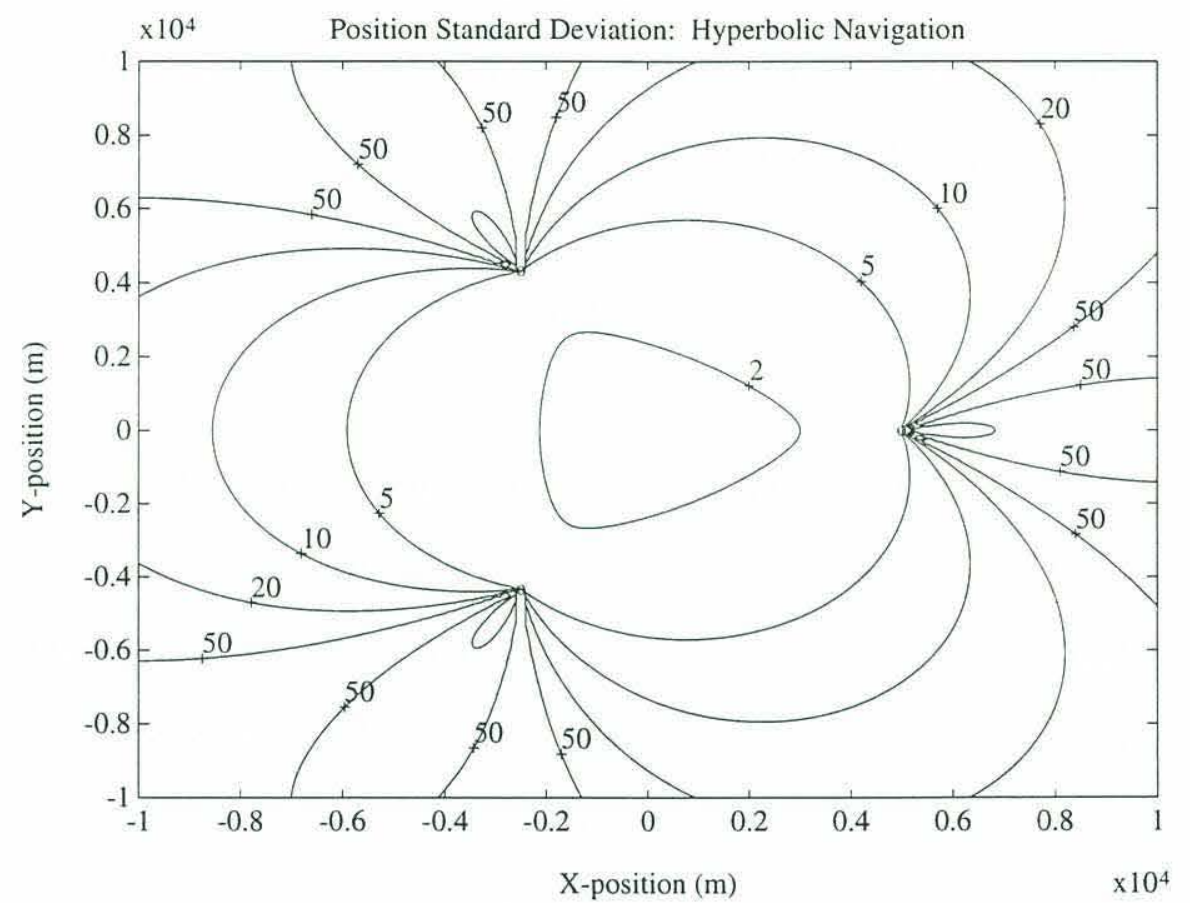

Figure 2.3: Standard deviation of position in meters for hyperbolic navigation with $1 \mathrm{~ms}$ standard deviation in travel time measurement.

The dominant feature of the error now is a large ridge behind each beacon. These ridges correspond to the places where the matrix $(M \partial f(\mathbf{x}) / \partial \mathbf{x})$ is nearly singular, or in other words, where the two hyperbola of position defined by the three beacons and vehicle depth are close to parallel. 


\section{CLOCK ERROR ESTIMATION}

The error in the vehicle clock with respect to beacon time was treated above as a deterministic but unknown constant added to the acoustic travel times. Hyperbolic navigation was presented as a way to remove this constant from the navigation equations. In this section, the clock error term will be treated as a random variable and will be incorporated into the noise. This view point will yield insight into the relationship between the hyperbolic and spherical navigation methods.

$$
\mathbf{t}=f(\mathbf{x})+(T+\mathbf{n})
$$

The Cramér-Rao bound is,

$$
\left(\frac{\partial f(\mathbf{x})^{T}}{\partial \mathbf{x}} \mathrm{E}\left[(T+\mathbf{n})(T+\mathbf{n})^{T}\right]^{-1} \frac{\partial f(\mathbf{x})}{\partial \mathbf{x}}\right)^{-1}
$$

It is assumed that the measurement noise and the clock error are uncorrelated and that the measurement noise is Gaussian, independent, and identically distributed from one beacon to the next. The vehicle clock error is Gaussian, but is the same for all beacons since the beacons all transmit at the same time. The bound can be rewritten,

$$
\begin{gathered}
\left(\mathrm{C}^{\mathrm{T}}\left(\sigma_{T^{2}} \mathbf{1}+\sigma_{R}{ }^{2} \mathrm{I}\right)^{-1} \mathrm{C}\right)^{-1} \\
\mathrm{C}=\frac{\partial f(\mathbf{x})}{\partial \mathbf{x}}, \quad \sigma_{R}^{2} \mathrm{I}=\mathrm{E}\left[\mathbf{n n}^{\mathrm{T}}\right], \quad \sigma_{T^{2}=E\left[T^{2}\right]}
\end{gathered}
$$

$\mathbf{1}$ is the matrix $\mathbf{1}_{i j}=1$

Clearly, when $T$ is known perfectly, $\sigma_{T}^{2}=0$, the bound is equivalent to the spherical navigation case. In the other limit, when $\sigma_{T}{ }^{2} \rightarrow \infty$, it is shown below that the bound is equivalent to the hyperbolic navigation case. A navigation system which 
estimates vehicle position and clock error together will then transition smoothly from hyperbolic to spherical performance as information is gained about $T$. Navigation accuracy could be improved in hyperbolic navigation systems where the beacons transmit at known time intervals simply by treating the transmission time as a random variable in this way.

To demonstrate the equivalence of the $\sigma_{T}{ }^{2} \rightarrow \infty$ case to hyperbolic navigation, it is observed that the noise covariance matrix is of a fortunate form where the inverse can be calculated easily. A solution to $\left(\sigma_{T}^{2} 1+\sigma_{R}^{2} \mathrm{I}\right)^{-1}$ is sought which has the form:

$$
\left(\sigma_{T^{2}} 1+\sigma_{R}^{2} \mathrm{I}^{-1} i j= \begin{cases}a & i=j \\ b & i \neq j\end{cases}\right.
$$

Because of the unusual symmetry of this matrix, the requirement that a matrix times its inverse yields the identity gives two equations that define $a$ and $b$. One equation corresponds to the diagonal terms of the identity, which must be one. The other corresponds to the off diagonal terms, which must be zero. If the size of the inverse matrix is $N \times N$,

$$
\begin{gathered}
\text { diagonal: }\left(\sigma_{T}^{2}+\sigma_{R}^{2}\right) a+(N-1) \sigma_{T}^{2} b=1 \\
\text { off-diagonal: } \quad \sigma_{T}^{2} a+\left(\sigma_{T}^{2}+\sigma_{R}^{2}\right) b+(N-2) \sigma_{T}^{2} b=0
\end{gathered}
$$

Solving for $a$ and $b$ gives,

$$
\begin{gathered}
a=\frac{1}{\sigma_{R}^{2}} \frac{(N-1) \sigma_{T^{2}}+\sigma_{R}^{2}}{N \sigma_{T}^{2}+\sigma_{R}{ }^{2}} \\
b=\frac{-1}{\sigma_{R}^{2}} \frac{\sigma_{T}^{2}}{N \sigma_{T}^{2}+\sigma_{R}^{2}}
\end{gathered}
$$


In the limit when $\sigma_{T}{ }^{2} \rightarrow \infty$, the noise covariance matrix inverse becomes,

$$
\begin{aligned}
& \lim _{\sigma_{T}^{2} \rightarrow \infty}\left(\sigma_{T}^{2} \mathbf{1}+\sigma_{R}^{2} \mathrm{I}\right)^{-1}=\left\{\begin{array}{cc}
\frac{1}{\sigma_{R}^{2}} \frac{N-1}{N} \quad i=j \\
\frac{1}{\sigma_{R}^{2}}-\frac{1}{N} \quad i \neq j
\end{array}\right. \\
& =\frac{1}{\sigma_{R}^{2}}\left(\mathrm{I}+\frac{-1}{N} \mathbf{1}\right)
\end{aligned}
$$

The Cramér-Rao Bound for the system that incorporates $T$ as a noise term, in the limit of $\sigma_{T^{2}} \rightarrow \infty$ is,

$$
\lim _{\sigma_{T}^{2} \rightarrow \infty} C R B_{T}=\sigma_{R}^{2}\left(\mathrm{C}^{\mathrm{T}}\left(\mathrm{I}+\frac{-1}{N} \mathbf{1}\right) \mathrm{C}\right)^{-1}
$$

Recall the bound for hyperbolic navigation,

$$
C R B_{H}=\left(\mathrm{C}^{\mathrm{T}} \mathrm{M}^{\mathrm{T}}\left(\mathrm{M} \sigma_{R}^{2} \mathrm{I} \mathrm{M}^{\mathrm{T}}\right)^{-1} \mathrm{M} \mathrm{C}\right)^{-1}
$$

The error covariance matrix part of this expression has the fortunate form of an identity matrix with a constant added to each element.

$$
\mathrm{M} \sigma_{R}^{2} \mathrm{I} \mathrm{M}^{\mathrm{T}}=\sigma_{R}^{2}(\mathrm{I}+1)
$$

An equation was just found for the inverse of matrices of this form. The matrix $\mathrm{MM}^{\mathrm{T}}$ is now $N-1 \times N-1$ where $\mathrm{R}$ was $N \times N$. 


$$
\begin{gathered}
\left(\mathrm{M} \sigma_{R}^{2} \mathrm{I} \mathrm{M}^{\mathrm{T}}\right)^{-1}=\left\{\begin{array}{c}
\frac{1}{\sigma_{R}^{2}} \frac{N-1}{N} \quad i=j \\
\frac{1}{\sigma_{R}^{2}} \quad \frac{-1}{N} \quad i \neq j
\end{array}\right. \\
=\frac{1}{\sigma_{R}^{2}}\left(\mathrm{I}+\frac{-1}{N} \mathbf{1}\right)
\end{gathered}
$$

Left Multiplying by $\mathrm{M}^{\mathrm{T}}$ and right multiplying by $\mathrm{M}$ gives,

$$
\begin{gathered}
\mathrm{M}^{\mathrm{T}}\left(\mathrm{M} \sigma_{R}{ }^{2} \mathrm{IM}^{\mathrm{T}}\right)^{-1} \mathrm{M}=\frac{1}{\sigma_{R}^{2}} \mathrm{M}^{\mathrm{T}}\left(\mathrm{I}+\frac{-1}{N} \mathbf{1}\right) \mathrm{M} \\
=\frac{1}{\sigma_{R}^{2}}\left(\mathrm{I}+\frac{-1}{N} \mathbf{1}\right)
\end{gathered}
$$

Thus, the Cramér-Rao bound for the hyperbolic system is

$$
C R B_{H}=\sigma_{R}^{2}\left(\mathrm{C}^{\mathrm{T}}\left(\mathrm{I}+\frac{-1}{N} \mathbf{1}\right) \mathrm{C}\right)^{-1}
$$

By comparison with equation (2.3.16), it is seen that,

$$
\lim _{\sigma_{T}^{2} \rightarrow \infty} C R B_{T}=C R B_{H}
$$

To illustrate this transition from hyperbolic accuracy to spherical accuracy as the variance of $T$ goes from infinity to zero, the Cramér-Rao bound for the standard deviation of position error magnitude is shown for several values of the standard deviation of $T$.

Figures 2.5-2.9 show the contours of the bound in a three-beacon network for the standard deviation of position when the standard deviation of measurement noise is $1 \mathrm{~ms}$. 
The bounds are shown for $\sigma_{T}=0,1 \mathrm{~ms}, 3 \mathrm{~ms}, 10 \mathrm{~ms}$, and $\infty$. Beacons and vehicle are in the same plane. The three beacons are shown as small circles.

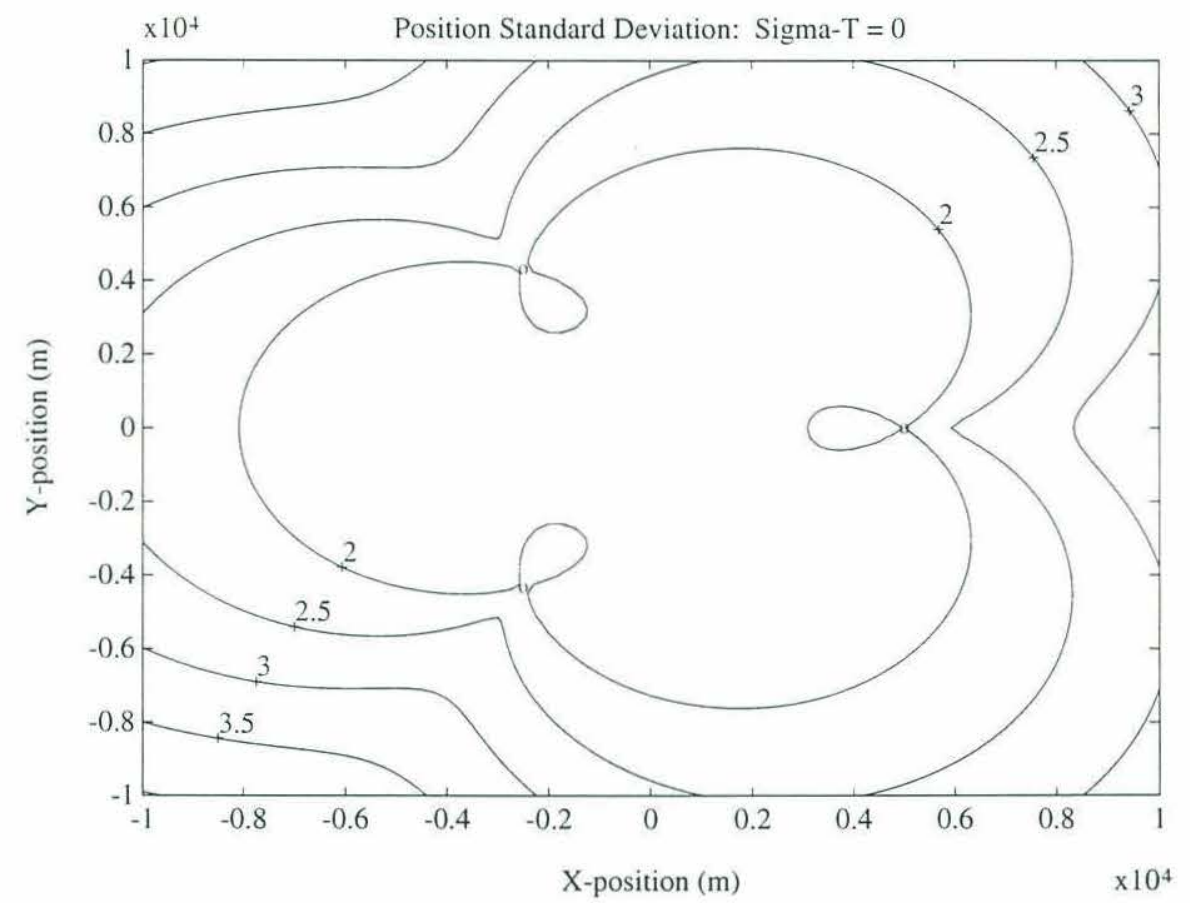

Figure 2.5: Standard Deviation of Position for $\sigma_{\mathrm{T}}=0$. This is equivalent to the spherical navigation system performance shown in Figure 2.3.

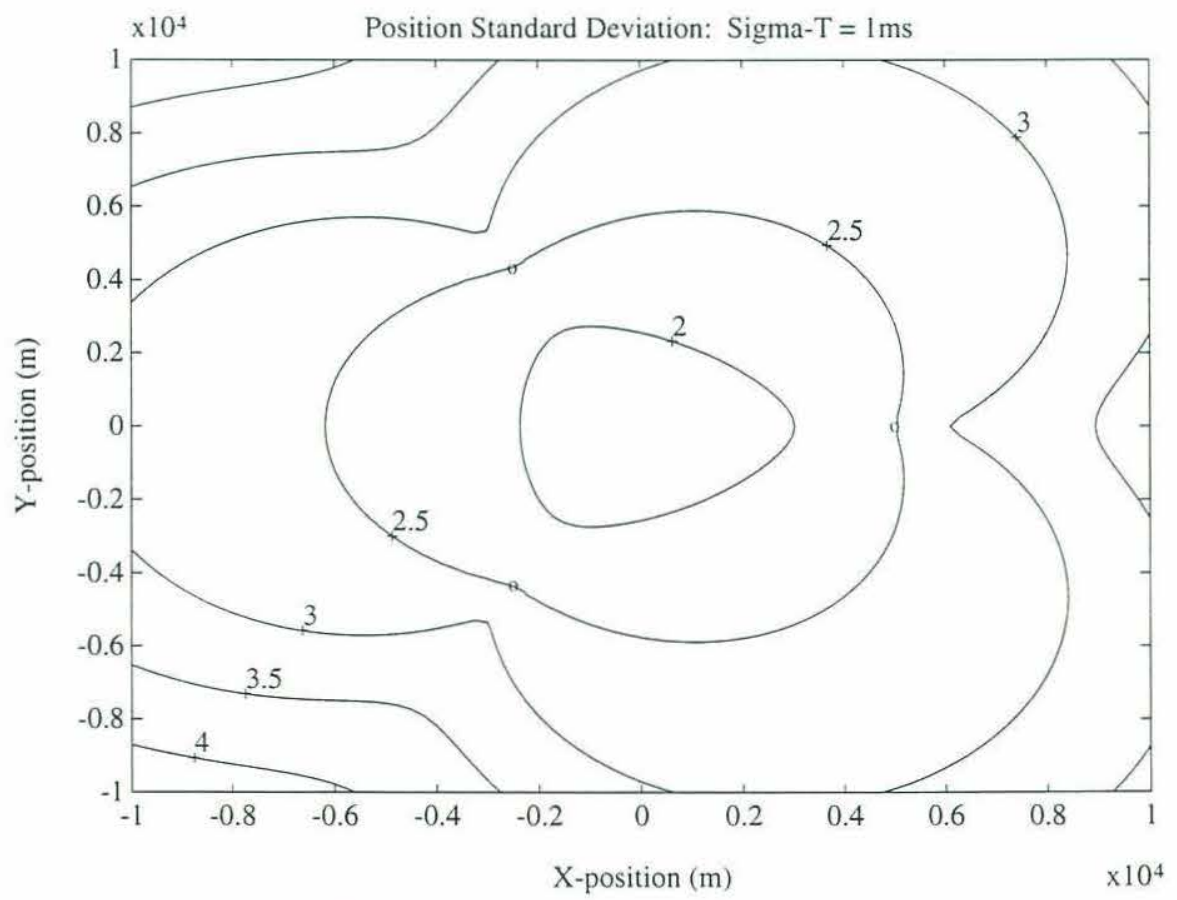

Figure 2.6: Standard Deviation of Position for for $\sigma_{\mathrm{T}}=1 \mathrm{~ms}$ 


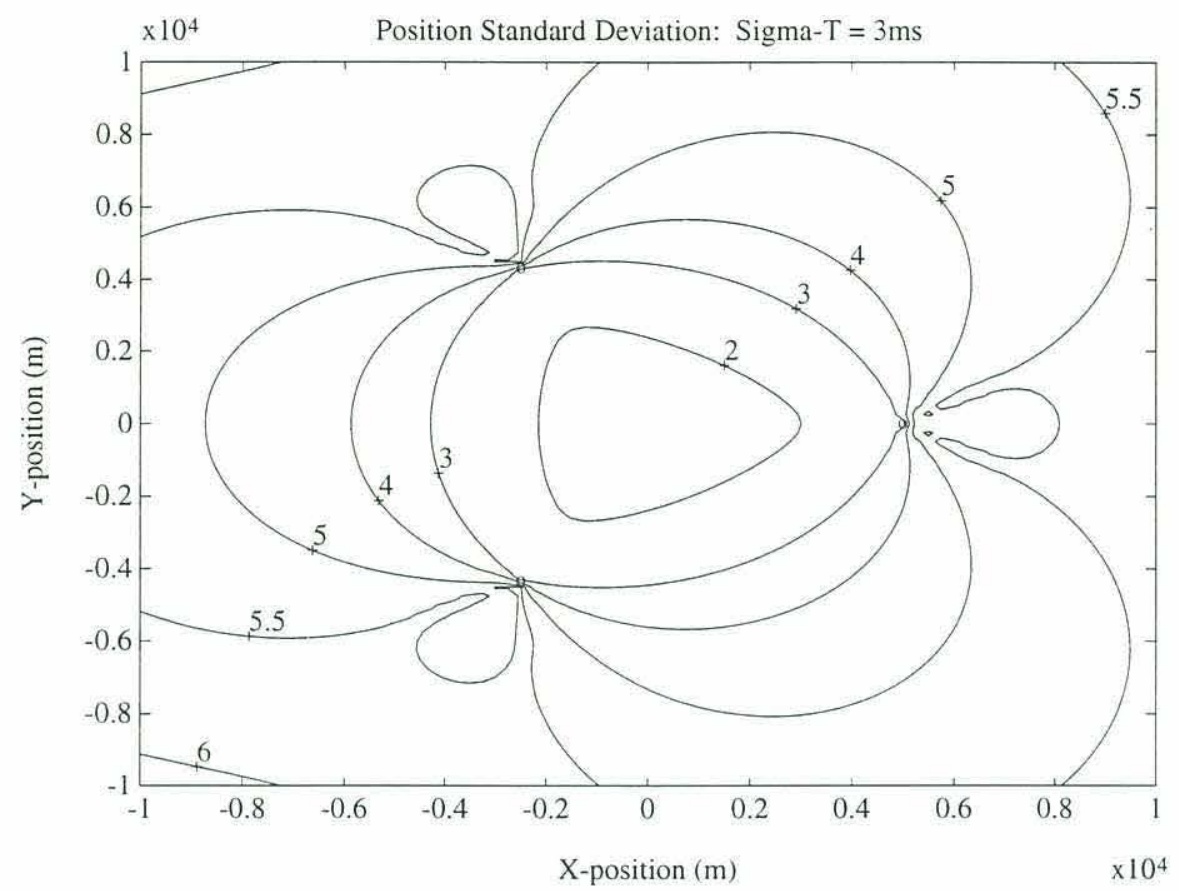

Figure 2.7: Standard Deviation of Position for $\sigma_{\mathrm{T}}=3 \mathrm{~ms}$

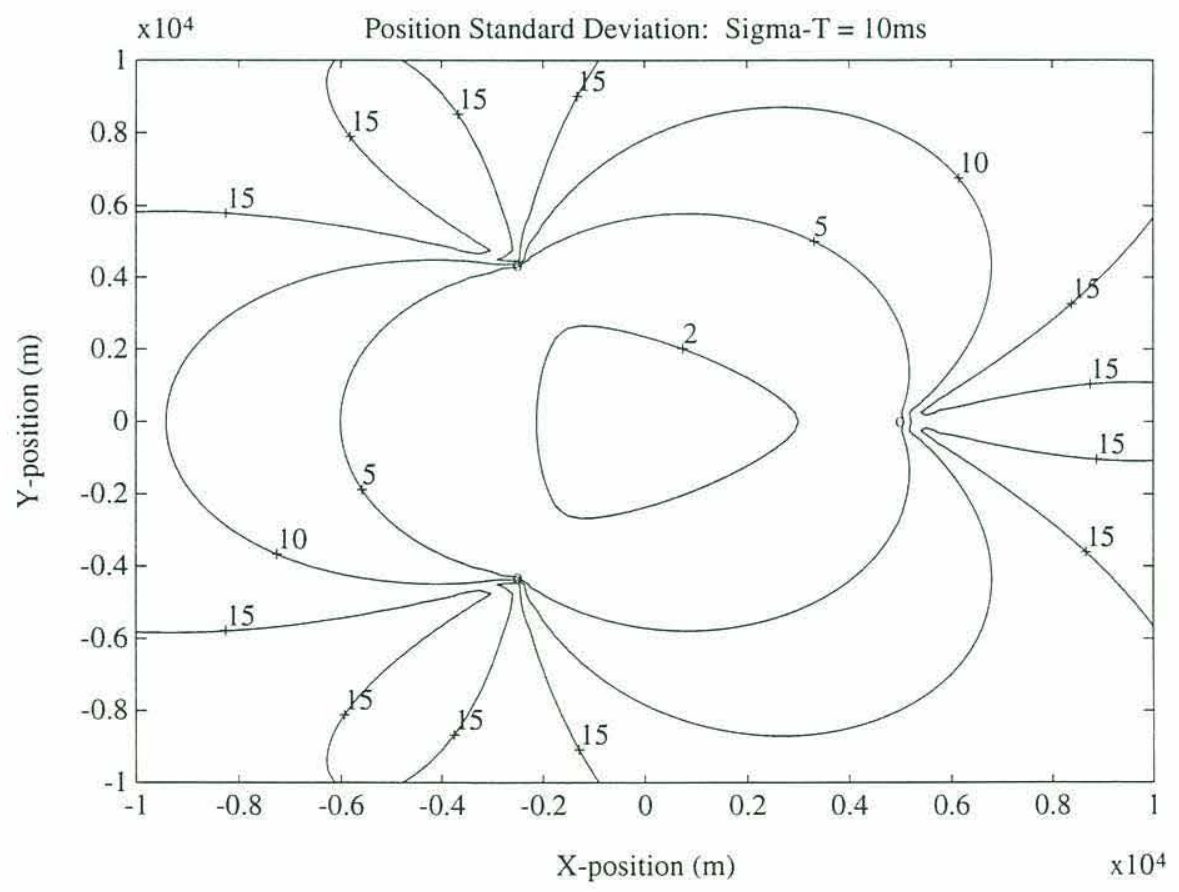

Figure 2.8: Standard Deviation of Position for $\sigma_{\mathrm{T}}=10 \mathrm{~ms}$ 


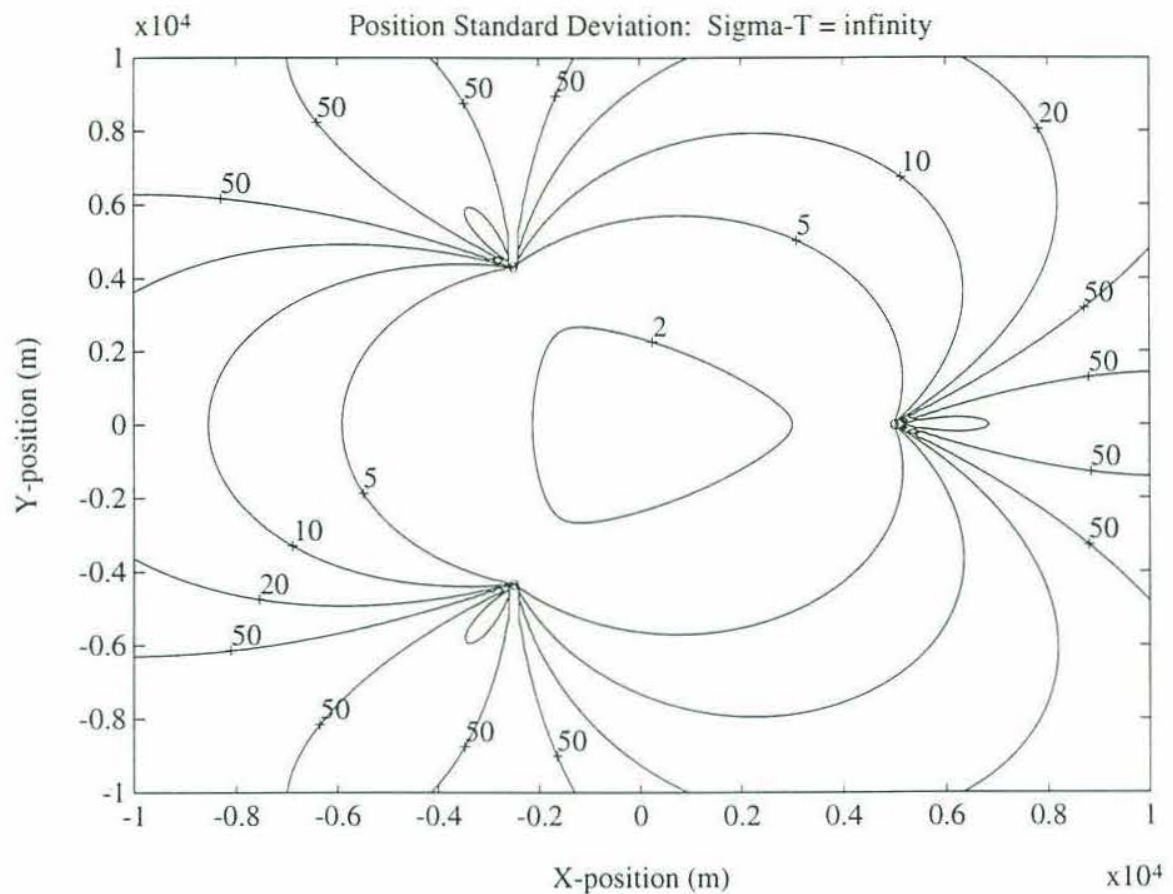

Figure 2.9: Standard Deviation of Position for $\sigma_{T}=\infty$. This is equivalent to the hyperbolic system performance shown in Figure 2.4. 


\section{SOUND SPEED PROFILE PARAMETERIZATION}

The sound speed profile is a continuous function of depth, but to estimate it, it must be reduced to some limited set of parameters. The changes in the sound speed profile are generally small variations about some mean profile. A convenient way to parameterize the profile, then, is to approximate the variations about the mean profile $c_{o}(z)$ in terms of the appropriate weightings $a_{n}$ applied to a set of basis functions $\phi_{n}(z)$ :

$$
c(z) \approx c_{O}(z)+\sum_{n=1}^{N} a_{n} \phi_{n}(z)
$$

If $N$, the number of functions necessary to achieve a satisfactory approximation of $c(z)$, is small enough, it will be possible to estimate the profile by estimating the parameters $a_{n}$.

The fewer number of parameters that are required to represent the profile satisfactorily, the easier (and usually the more accurate) the approximation. What, then, is the optimal basis to choose so that the profile can be represented with a minimum number of basis functions? To answer this question, it is first necessary to define some measure of how "good" a particular representation of the profile is. A popular measure is the $\mathrm{L}^{2}$ norm, under which the choice of functions can be easily calculated [Davis, 1976]. These functions are generated from a sample set of profiles, minimizing the error in their representation of the sample set. Hence they are known as empirical orthogonal functions, or EOFs.

The EOFs are chosen in such a way as to minimize the variance of the error in their . representation of a sample set of sound velocity profiles. First, the mean profile is subtracted from the set, leaving a set of variations about the mean $\mathbf{c}_{\mathbf{n}}$. The variation for each profile is approximated by a (normalized) basis function $\phi$ weighted appropriately by $\alpha_{n}$. The error in this approximation can be written, 


$$
\mathbf{c}_{n}-\phi \alpha_{n}=\varepsilon_{n}
$$

where, $\alpha_{n}=\left(\mathbf{c}_{n}{ }^{T} \phi\right)$

The goal is to minimize the total error over all the sample profiles,

$$
\begin{gathered}
\sum_{\mathrm{n}} \varepsilon_{\mathrm{n}}^{\mathrm{T}} \varepsilon_{\mathrm{n}} \\
=\sum_{\mathrm{n}}\left(\mathbf{c}_{\mathrm{n}}-\phi\left(\mathbf{c}_{\mathrm{n}}^{\mathrm{T}} \phi\right)\right)^{\mathrm{T}}\left(\mathbf{c}_{\mathrm{n}}-\phi\left(\mathbf{c}_{\mathrm{n}}^{\mathrm{T}} \phi\right)\right) \\
=\sum_{\mathrm{n}} \mathbf{c}_{\mathrm{n}}^{\mathrm{T}} \mathbf{c}_{\mathrm{n}}-\mathbf{c}_{\mathrm{n}}^{\mathrm{T}} \phi \mathbf{c}_{\mathrm{n}}^{\mathrm{T}} \phi-\phi^{\mathrm{T}} \mathbf{c}_{\mathrm{n}} \phi^{\mathrm{T}} \mathbf{c}_{\mathrm{n}}+\phi^{\mathrm{T}} \mathbf{c}_{\mathrm{n}} \phi^{\mathrm{T}} \phi \mathbf{c}_{\mathrm{n}}^{\mathrm{T}} \phi
\end{gathered}
$$

$\phi^{\mathrm{T}} \phi=1$ since the basis functions $\phi$ are normalized. The term $\mathbf{c}_{\mathrm{n}}^{\mathrm{T}} \mathbf{c}_{\mathrm{n}}$ is independent of the minimization. So this expression condenses to,

$$
\min (|\phi|=1) \sum_{\mathrm{n}}-\mathbf{c}_{\mathrm{n}}^{\mathrm{T}} \phi \mathbf{c}_{\mathrm{n}}^{\mathrm{T}} \phi
$$

Or equivalently,

$$
\max (|\phi|=1) \sum_{\mathrm{n}} \mathbf{c}_{\mathrm{n}} \mathrm{T}_{\phi} \mathbf{c}_{\mathrm{n}} \mathrm{T}_{\phi}
$$

Let the matrix $\mathrm{C}$ have as its columns the profile variations $\mathbf{c}_{n}$ for the $N$ sample profiles. The maximization is then,

$$
\begin{gathered}
\max (|\phi|=1)\left(\mathrm{C}^{\mathrm{T}} \phi\right)^{\mathrm{T}} \mathrm{C}^{\mathrm{T}} \phi \\
\max (|\phi|=1) \phi^{\mathrm{T}} \mathrm{C} \mathrm{C}^{\mathrm{T}} \phi
\end{gathered}
$$


The matrix $\mathrm{C} \mathrm{C}^{\mathrm{T}}$ is positive definite, and the solution to this maximization is that $\phi$ is the eigenvector of the largest eigenvalue of $\mathrm{C} \mathrm{C}^{\mathrm{T}}$.

That provides the first EOF. To produce the second and subsequent EOFs, the observation is made that the matrix $\mathrm{CC}^{\mathrm{T}}$ is Hermetian and thus its eigenvectors are orthogonal. The basis function which represents the next largest amount of variation in $\mathrm{CC}^{\mathrm{T}}$ is therefore the eigenvector with the second largest eigenvalue, and so forth. The best $N$ basis functions to choose to represent the greatest portion of the variance are the eigenvectors corresponding to the $N$ largest eigenvalues.

A set of ten sample profiles was used to generate the basis functions. These profiles are shown in Figure 2.9. They were measured over a period of five days at twelve hour intervals during the CEAREX ' 89 experiment in the Greenland Sea [Scheer].

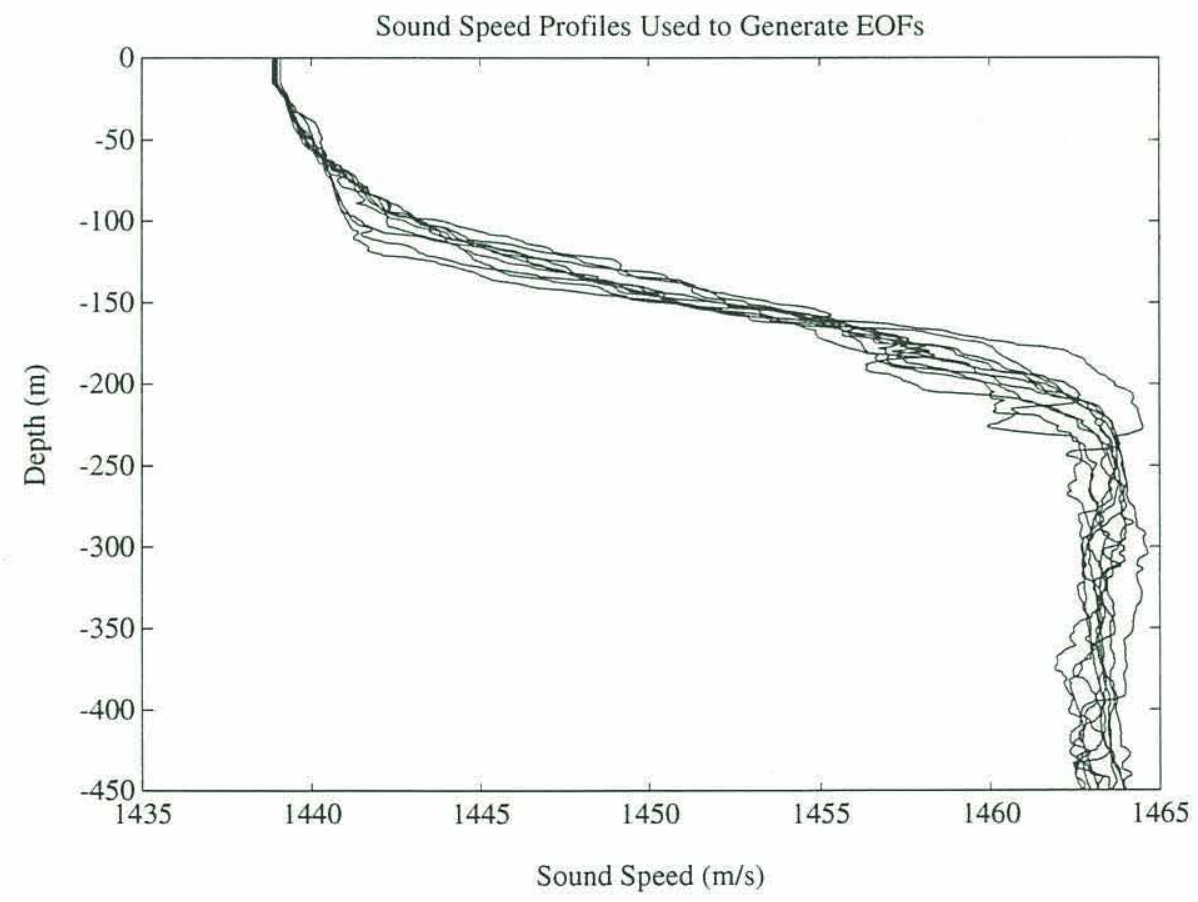

Figure 2.10: Set of ten sample profiles used to generate the EOFs. These profiles were taken in the Greenland Sea over a period of five days. 

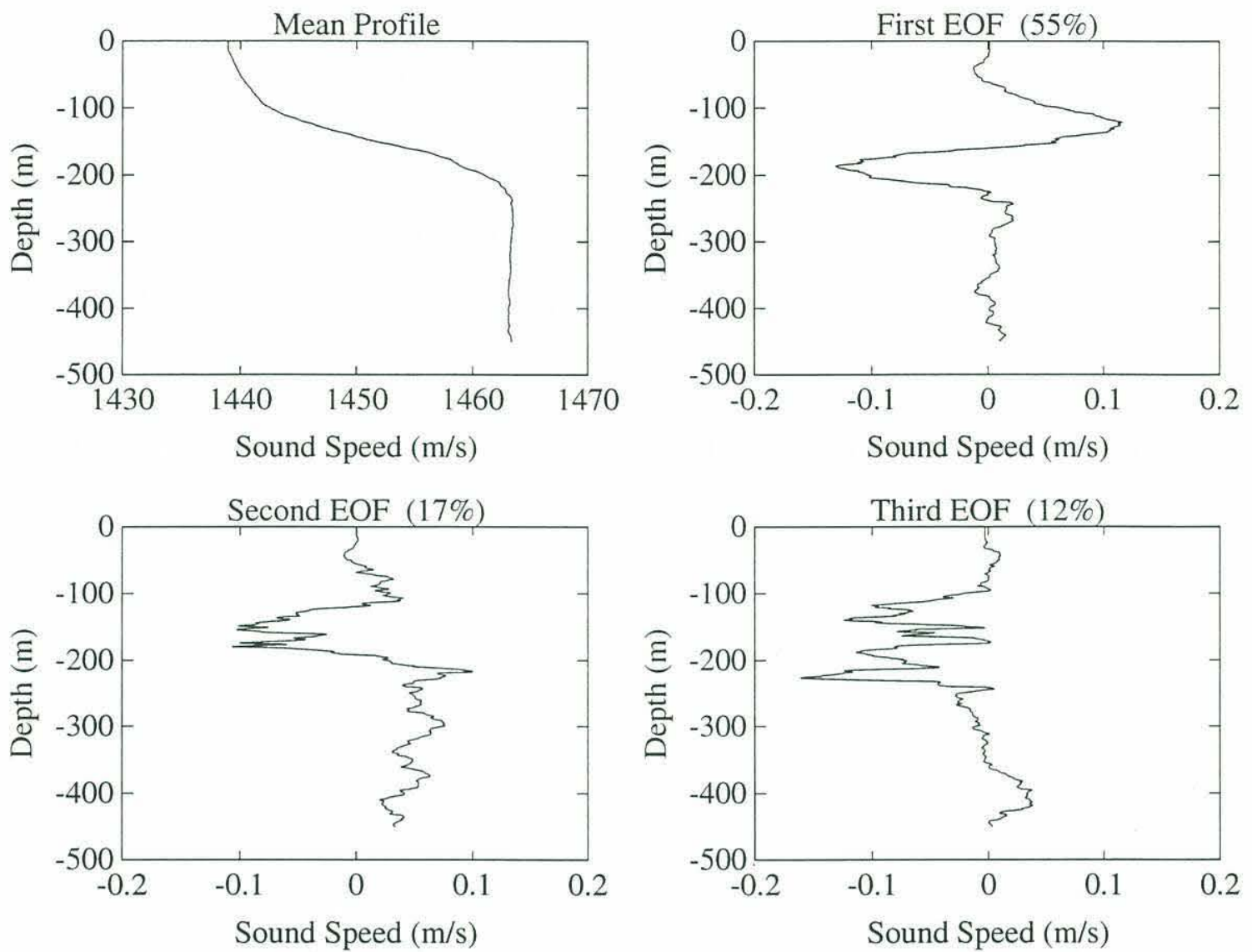

Figure 2.11: The mean profile and the first three EOFs corresponding to the set of ten profiles in Figure 2.10. The percentage of the total variance which each EOF represents is given in parentheses.

The Cramér - Rao bound can be found for the variance of sound speed profile parameter estimates. Assume for now that vehicle position and clock synchronization are known. The case of estimating the sound speed profile when position and clock synchronization are also unknown will be considered in Chapter 4. At a given vehicle position,

$$
\mathbf{t}=g(\mathbf{p})+\mathbf{n}
$$

The vector of sound speed profile parameters is $\mathbf{p}$, which produces changes in travel times through the function $g$. The noise $\mathbf{n}$ is assumed to be Gaussian, with a covariance of $\mathbf{R}$. The Cramér-Rao bound on estimation of $\mathbf{p}$ becomes, 


$$
C R B_{p}=\left(\frac{\partial g(\mathbf{p})^{\mathrm{T}}}{\partial \mathbf{p}} \mathrm{R}^{-1} \frac{\partial g(\mathbf{p})}{\partial \mathbf{p}}\right)^{-1}
$$

The noise $\mathbf{n}$ is taken to have a standard deviation of $1 \mathrm{~ms}$. The medium is assumed to have the Arctic sound speed profile given in Figure 1.3 so that there are multiple ray paths through the medium. The source and receiver are at $30 \mathrm{~m}$ depth. The bound on the standard deviation of the profile parameter for the first EOF in Figure 2.11 is shown in Figure 2.12.

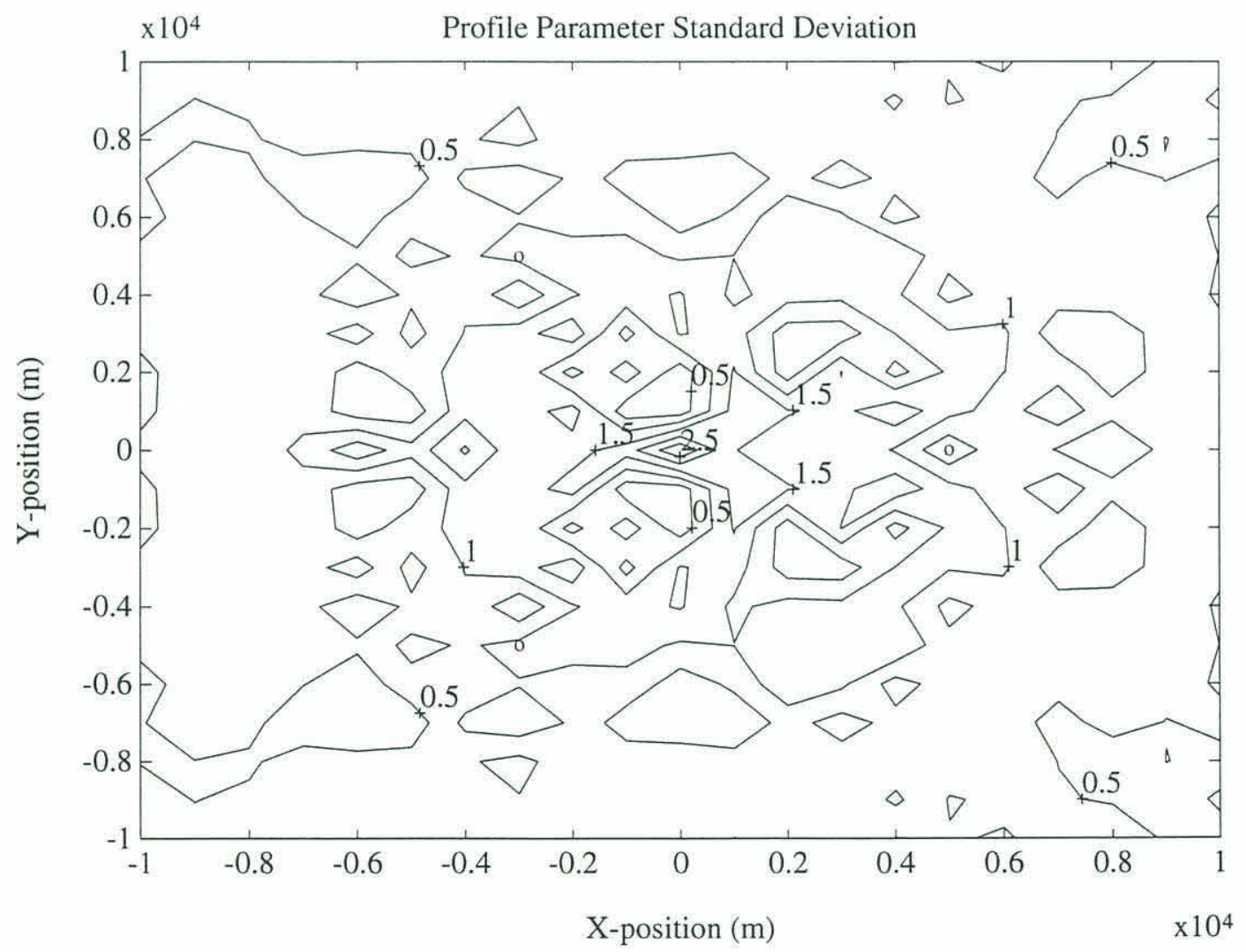

Figure 2.12: Standard Deviation of the estimate of the weight of the first EOF in figure 2.11.

This graph illustrates some interesting behavior. First, the size of the error tends to decrease as beacon range increases. At longer ranges there is more multipath, which means the sound speed profile is sampled more densely by the rays. In the center of the beacon 
network is the global maximum of error. Here the range from each beacon is the same, so each ray path exists in triplicate and the identical ray geometries from each beacon provide redundant information about the profile. The longer the range over which a sound speed profile perturbation is observed, the greater its effect on travel time, and the more observable it will be. This effect would also contribute to the poorest approximation at the center of the array.

The sound speed profile parameter estimate is most accurate when the set of rays at a particular range sample the basis function most sensitively. Toward this end, the sampling density of a ray path is defined. Let $d(z)$, the sampling density of a particular ray, relate the slowness profile $1 / \mathrm{c}(\mathrm{z})$ to the acoustic travel time $\mathrm{t}$,

$$
t=\int_{0}^{\infty} d(z) \frac{1}{c(z)} d z
$$

The sampling density is found by comparison of equation 2.3 .31 to the ray time integral [Frisk, 1994],

$$
t=\int_{\text {Zinitial }}^{\text {Zfinal }} \frac{1}{\sin \theta(z)} \frac{1}{c(z)} d z
$$

Using Snell's Law, $\cos \theta(z)=c(z) / c_{t}$, where $c_{t}$ is the sound speed at which the ray becomes horizontal,

$$
d(z)=\frac{k}{\sqrt{1-\frac{c^{2}(z)}{c_{t}^{2}}}}
$$

where $k$ is the number of times the ray passes depth $z$. 

that $c(z)=c_{0}(z)+\partial c(z)$ can be written,

$$
\Delta t=\left(\frac{1}{c_{0}(z)+\partial c(z)}\right) d(z)-\left(\frac{1}{c_{0}(z)}\right) d(z)
$$

which for small variations about $\mathrm{c}_{0}(\mathrm{z})$, can be approximated as,

$$
\Delta t \approx-\partial c(z) \frac{d(z)}{c_{0}(z)^{2}}
$$

A computational side note on the sampling density is in order here. Since the sampling density has a singularity at the turning depth of the ray, the discrete approximation of $d(z)$ as $d_{n}=d(n \Delta z)$ is poor in the neighborhood of the discontinuity. Since $d(z)$ is a sampling density, it is important that the summed area under the discrete function be as close as possible to the integrated area under the continuous function. A better choice for $d_{z}$ is then:

$$
d_{z}=\int_{q}^{(q+1) \partial z} \frac{k}{\sqrt{1-\frac{c_{p}^{2}(z)}{c_{t}^{2}}}} d z
$$

The sound speed $c_{p}(z)$ is taken to be linear over the region of integration, so $c_{p}(z)=c_{O}+$ $\beta z$. Performing the change of variables from $z$ to $c$ gives: 


$$
\begin{gathered}
d_{z}=\int_{c(q \partial z)}^{c((q+1) \partial z)} \frac{k}{\beta \sqrt{1-\frac{c_{p}^{2}}{c_{t}^{2}}}} c_{p} \\
=\frac{k}{a \beta}\left(\operatorname{asin}\left(a c_{q+1}\right)-\operatorname{asin}\left(a c_{q}\right)\right) \\
t=\sum_{z=0}^{Z} \frac{1}{c_{z}} d_{z}, \quad \Delta t=\sum_{z=0}^{Z}-\frac{\partial c_{\mathrm{Z}}}{c_{z}^{2}} d_{z}
\end{gathered}
$$

A ray path and the corresponding sampling density are shown in Figure 2.13. The ray samples the water column most sensitively at its turning depth. The ray path chosen for this example connects a source and receiver at $30 \mathrm{~m}$ depth with a horizontal range of $3 \mathrm{~km}$. The sound speed profile is that of Figure 1.3.

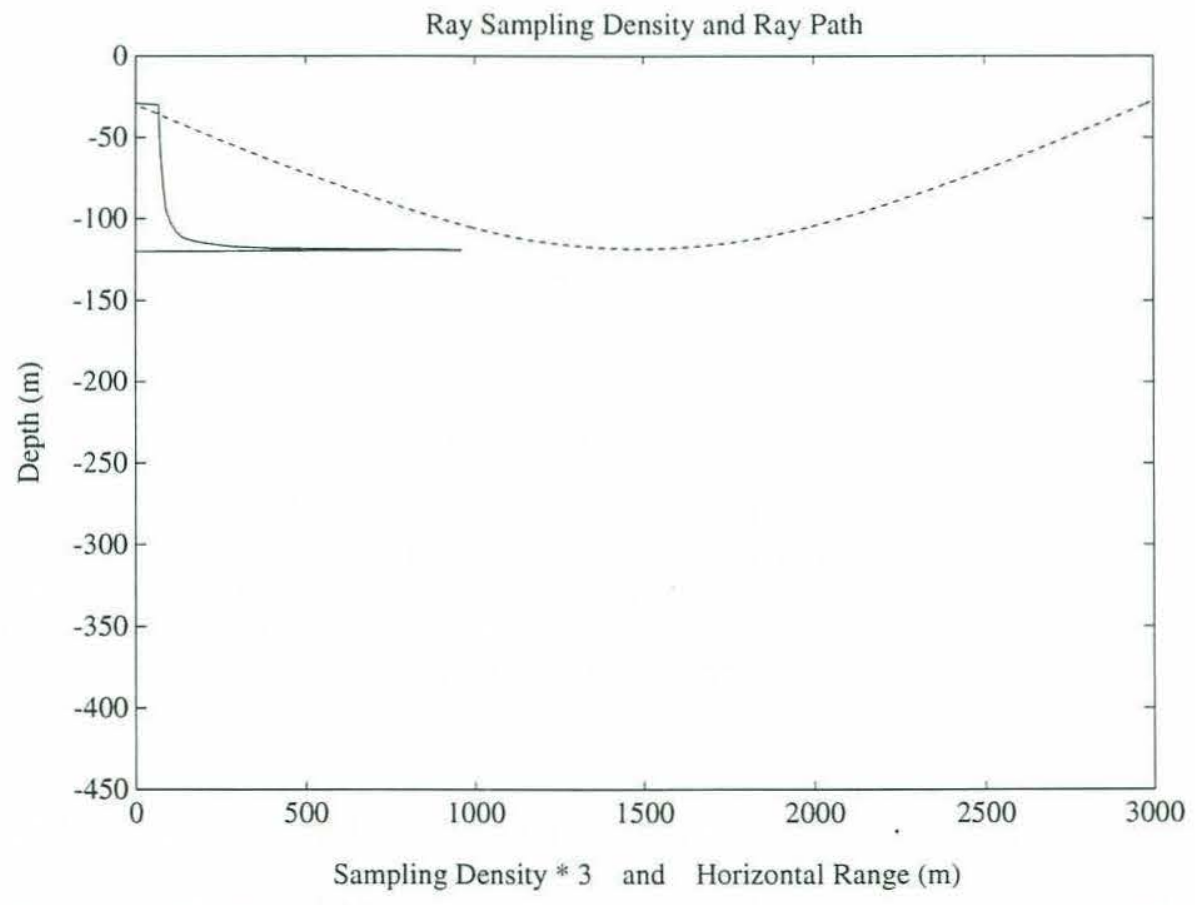

Figure 2.13: Ray sampling density (solid line) and ray path (dashed line). The ray sampling density has been multiplied by three to fit onto the same axes as the ray. 
In Figure 2.12, the standard deviation in the estimate of the first EOF coefficient was seen to have a global maximum at the center of the navigation network $(0,0)$. A region of low error was located about $1000 \mathrm{~m}$ above the center at $(0,1000)$. The ray sampling densities at these two locations explain the dramatic differences in error performance between the two locations. Figure 2.14 shows the ray sampling densities for all the eigenrays at location $(0,0)$ superimposed on the first EOF. The spikes in the sampling correspond to the depths at which the ray travel time is most sensitive to a sound velocity change.

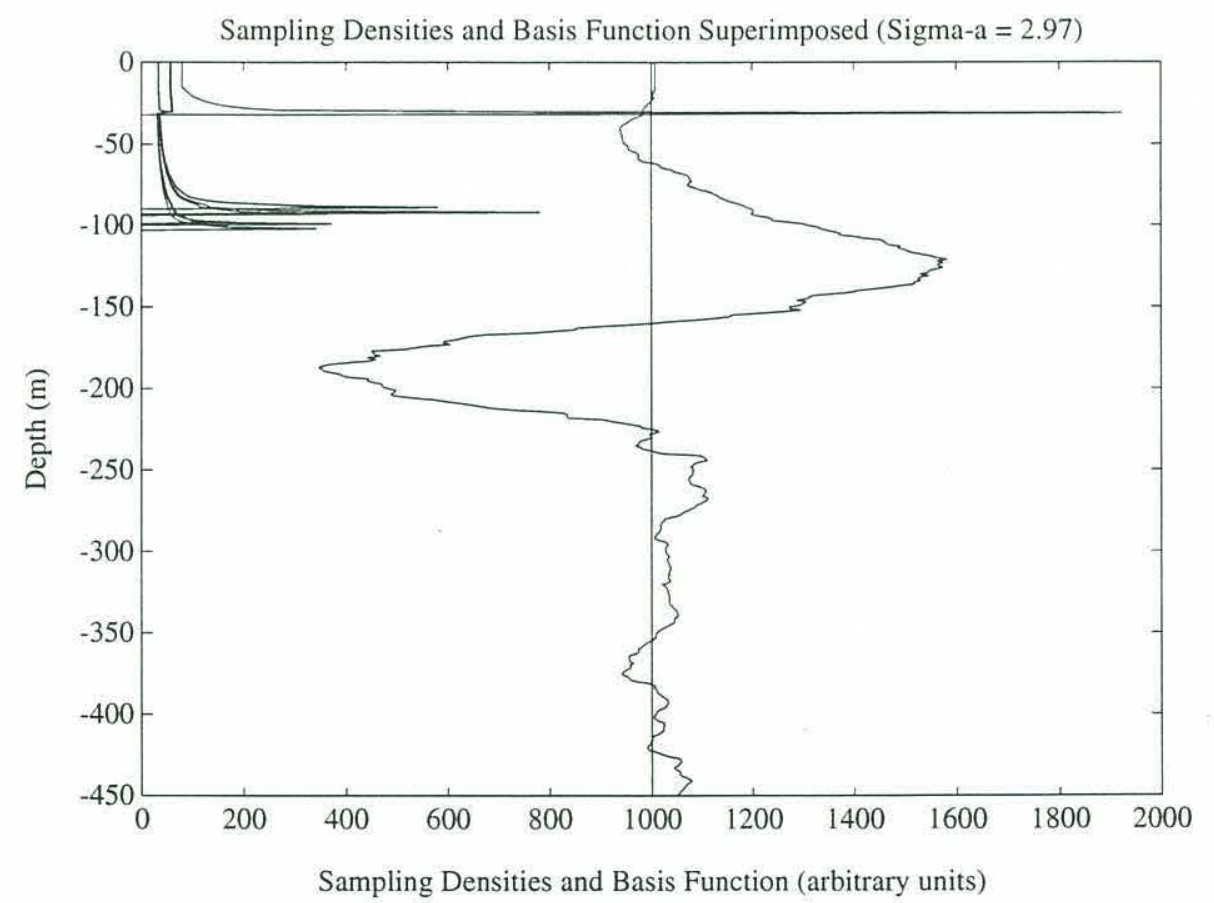

Figure 2.14: Ray Sampling Densities and the first EOF from Figure 2.11 at location $(0,0)$ in Figure 2.12. This location is in a region of high estimation error for the weight of the first EOF. This is reflected by the sparseness with which the rays sample the depths where the EOF is largest.

Figure 2.15 shows the ray sampling densities for all the eigenrays at location $(0,1000)$ superimposed on the first EOF. The sensitivity to the first EOF in Figure 2.15 is clearly much greater than in Figure 2.14. 


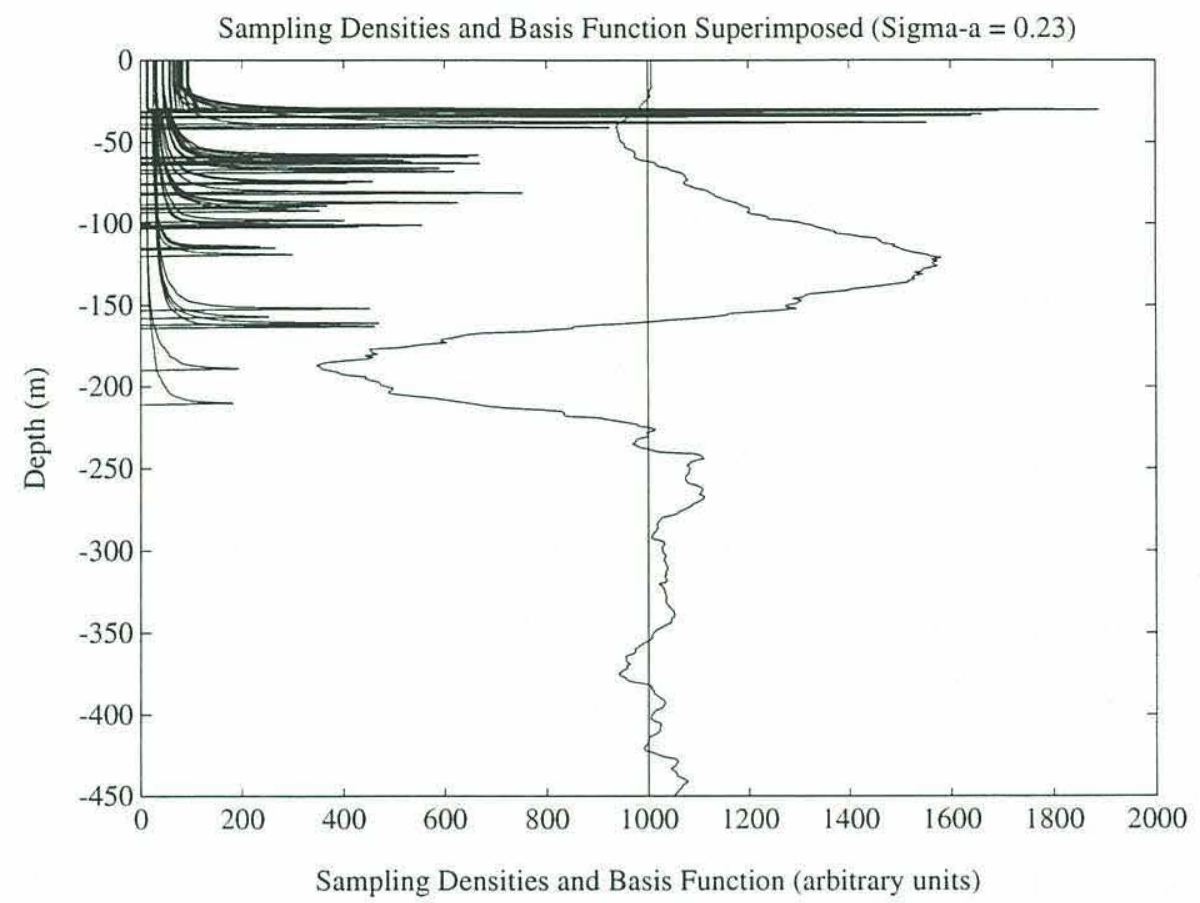

Figure 2.15: Ray Sampling Densities and the first EOF from Figure 2.11 at location $(0,1000)$ in Figure 2.12. This location is in a region of low estimation error for the weight of the first EOF. This is reflected by the large number of ray paths which sample sensitively the depths where the EOF is largest.

The appropriate weights for the EOFs are estimated most accurately when the eigenrays for a particular source-receiver geometry sample most sensitively the depths where the EOFs are largest. 


\subsection{UNCERTAINTY BOUNDS}

Now that the parameter choice has been established, the size of parameter uncertainty will be examined.

\section{POSITION UNCERTAINTY BOUND}

A sense of the sizes of navigation errors which can be caused by path fading can be had through a simple calculation. Path fading introduces errors by causing misidentification of arrivals. If the span of arrival times for all rays at a given range can be bounded, then the time errors corresponding to mistaking one ray for another can be bounded as well. Toward this end, the effective horizontal velocity of a ray is defined as the horizontal range divided by the travel time. The span of possible travel times for a given horizontal range from the beacon can be found by calculating the maximum and minimum possible horizontal velocity for a given ray. The horizontal velocity of the ray at any position is $c(z) \cos \theta(z)$. By Snell's law, this can be rewritten:

$$
c_{h}(z)=a c^{2}(z)
$$

The maximum value for $c_{h}(z)$ occurs when the ray is horizontal, or $c(z)=1 / a$, as this is the fastest water which the ray can enter and the ray is moving horizontally.

$$
\max c_{h}(z)=\frac{1}{a}
$$

In the Arctic, the lowest sound speed usually occurs at the surface $z=0$.

$$
\min c_{h}(z)=a c^{2}(0)
$$


For beacons at the surface (dashed line) and at 100m depth (solid line), the maximum and minimum horizontal velocity are shown as a function of ray shooting angle. These bounds are based on the surface and $100 \mathrm{~m}$ sound speed values of the Arctic sound speed profile in Figure 1.3. The bound is normalized with respect to the sound speed at the transmitter depth.

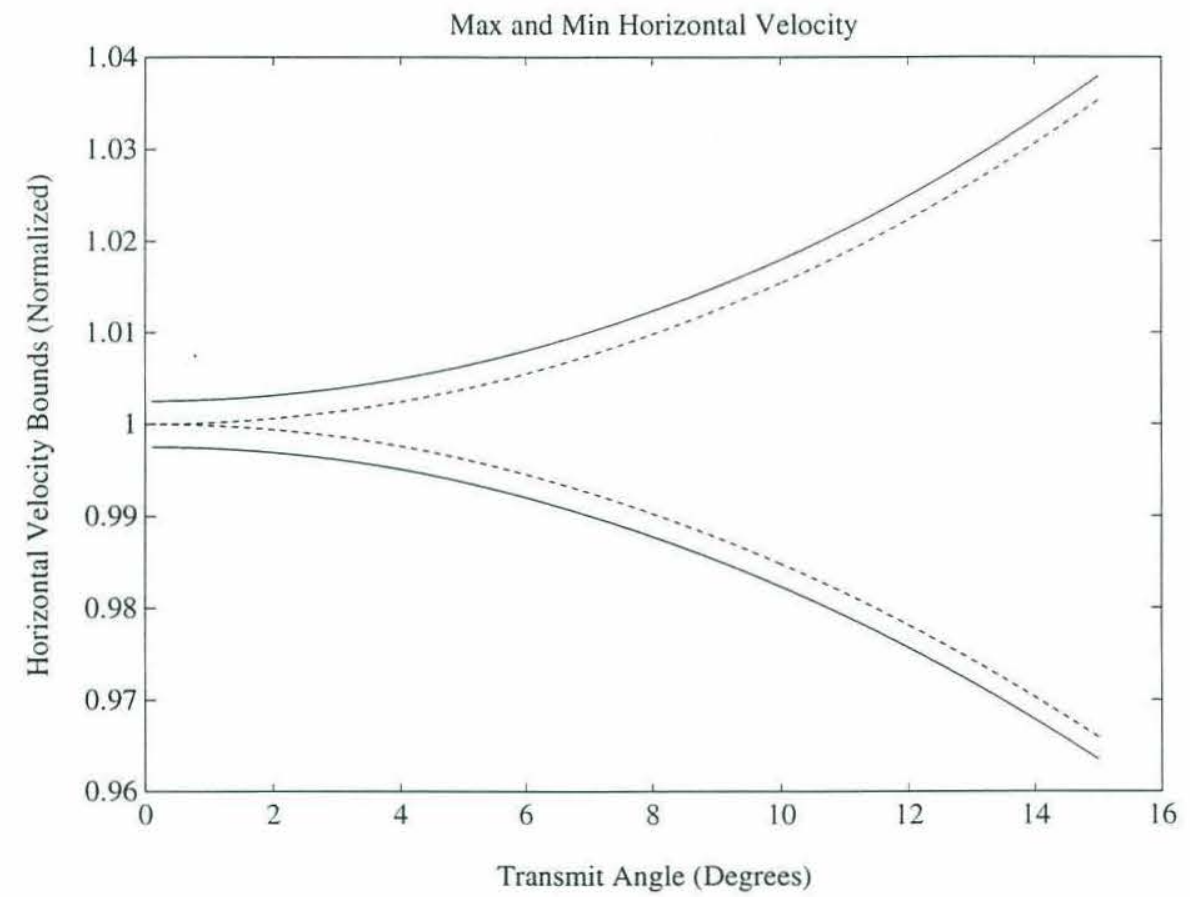

Figure 2.16: Bound on horizontal ray velocity for $0 \mathrm{~m}$ (dashed line) and $100 \mathrm{~m}$ (solid line) transmitter depths. The bound is normalized with respect to the sound speed at the transmitter depth.

These bounds demonstrate that for a shallow source and receiver, where the ray shooting angle is small, the span of horizontal velocities is small, amounting to only a few percent. This small uncertainty in effective horizontal velocity translates into a small uncertainty in path fading induced range error. The uncertainty in range will be only a few hundred meters at $10 \mathrm{~km}$. 
The clock error between the vehicle clock and the acoustic beacons, which may be mutually synchronized to GPS time, was treated as a random variable in Section 2.3. A typical drift rate for a vehicle using an uncompensated crystal oscillator as its time base is one part in $10^{4}$ [Horowitz and Hill, 1989].

\section{SOUND SPEED PROFILE UNCERTAINTY}

The magnitude of uncertainty in the sound speed profile is not as easy to bound, but a sense of the size of variations to be expected can be had by examining a sample set of profiles. The maximum basis function weight and the standard deviation of weights is calculated for the three EOFs found in the previous section. These values are based on the ten profiles used to generate the EOFs.

$\begin{array}{ccc}\text { Basis Function } & \text { Maximum Weight } & \begin{array}{c}\text { Weight Standard } \\ \text { Deviation }\end{array} \\ \phi_{1} & 26 & 13 \\ \phi_{2} & 21 & 14 \\ \phi_{3} & 12 & 6\end{array}$

Next the weights were found for the subsequent ten profiles in the time series. It is interesting to note that the dominant modes of variation have changed. Basis function $\phi_{3}$ has gone from being the least significant to being the most significant mode.

$\begin{array}{ccc}\text { Basis Function } & \begin{array}{c}\text { Maximum Weight } \\ \begin{array}{c}\text { Weight Standard } \\ \text { Deviation }\end{array}\end{array} \\ \phi_{1} & 33 & 16 \\ \phi_{2} & 43 & 23 \\ \phi_{3} & 76 & 36\end{array}$


This change in the dominant mode of the sound speed profile variation suggests that the profile variation is not a stationary process over the collection of profiles used. EOFs are optimized under the assumption of stationarity, so their effectiveness as a modeling tool may be somewhat reduced. 


\subsection{SIGNATURE MATCHING NAVIGATION}

With a parameter set and measured quantities defined, the application of a signaturematching approach to multipath navigation is now considered. Such an approach requires a "goodness of match" energy function defined over the parameter space, the maximum of which corresponds to the correct parameter choice. Such an energy function is defined as follows. First, a predicted signal density is created by replacing each predicted point in arrival angle - travel time space with a Gaussian weighting function with variances of $1 \mathrm{~ms}$ in travel time and 1 degree in arrival angle. An energy function describing the goodness of match between a predicted and measured signal can be created by summing over the measured arrivals the predicted signal density at each measured arrival location. The parameter choice producing the predicted signal that maximizes this energy function is taken to be correct. This energy function is plotted below for a beacon at $7 \mathrm{~km}$ range. Range variation is shown in the direction into the page, and sound speed profile parameter variation is horizontal. 


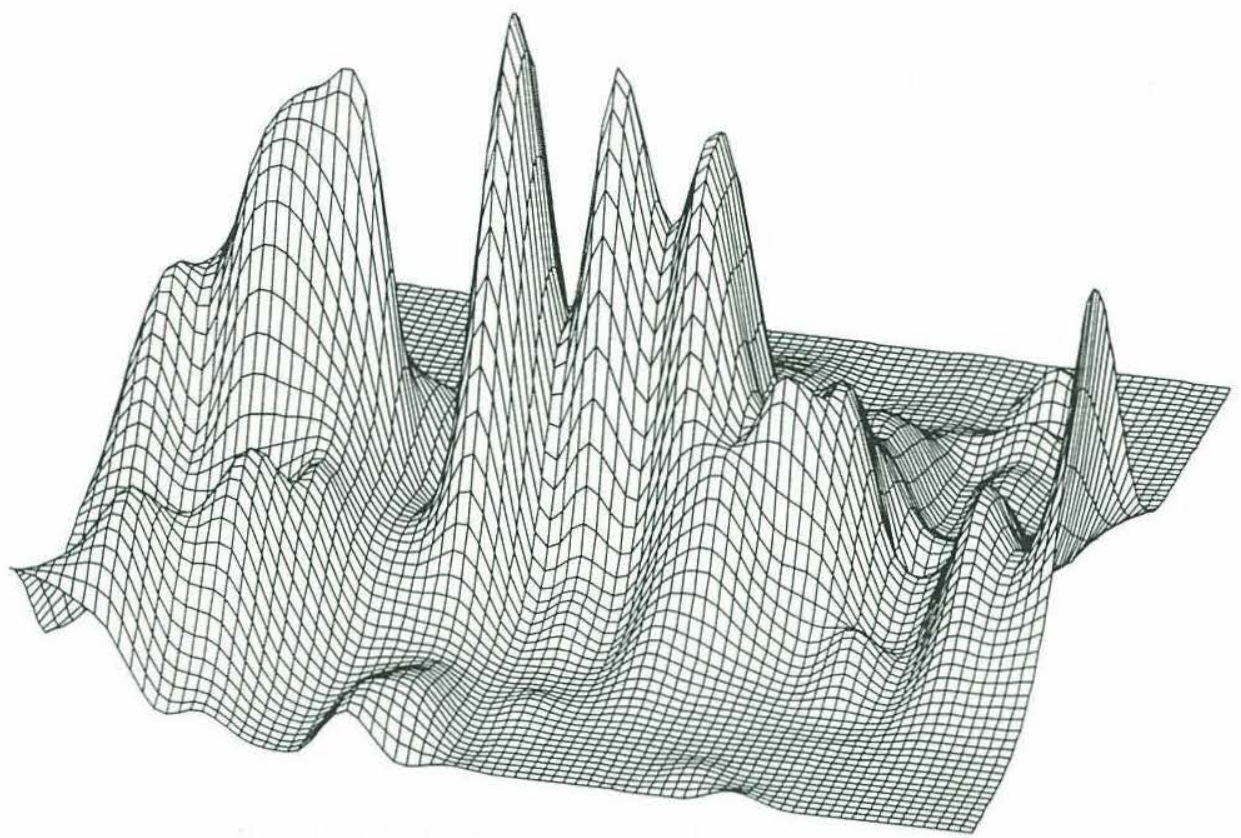

Figure 2.17: An energy function describing the "goodness" of match between the predicted and measured signals. The horizontal axis is the weight of the first EOF and it ranges from -40 to 40 . The axis into the page is beacon range, and it varies from -7 to 7 meters around the true range of $7000 \mathrm{~m}$. The optimal parameter choice would correspond to the maximum of this function.

On such a surface, finding a global maximum would be exceedingly difficult. A ray-based approach can improve the situation greatly. Each spike of this energy function corresponds to an intersection of one or more predicted and measured arrivals. By explicitly matching arrivals rather than searching an energy surface, all of the maxima can be found directly, and then the largest one may be selected. 


\subsection{LINEAR APPROXIMATION}

A ray-based approach to multipath navigation requires relations between parameter shifts and travel time and angle shifts. In this section, the behavior of the measurable quantities of travel time and arrival angle over the regions of parameter uncertainty will be investigated. The derivatives of each of the measurable quantities with respect to each parameter are calculated in Appendix B.

\section{VARIATION OF TRAVEL TIME WITH RANGE}

The variation of travel time with range is shown in Figure 2.18 for a source and receiver at $30 \mathrm{~m}$ depth. So that arrival spacing can be seen on one graph for all ranges, reduced time, $T_{r}$, is plotted. Reduced time is defined as the travel time $T$ with a factor proportional to range $R$ subtracted.

$$
T_{r}=T-R / 1450
$$

In Figure 2.19, the reduced time is again plotted against range but for a sound speed profile taken 24 hours later. Notice that new arrivals have appeared at about $7 \mathrm{~km}$ range, and the arrivals that were at $6 \mathrm{~km}$ range have faded.

The important feature of both of Figure 2.18 and 2.19 is that they can be closely approximated as consisting of disjoint linear segments. As long as a particular segment exists between two ranges, the travel time will vary linearly with range. 


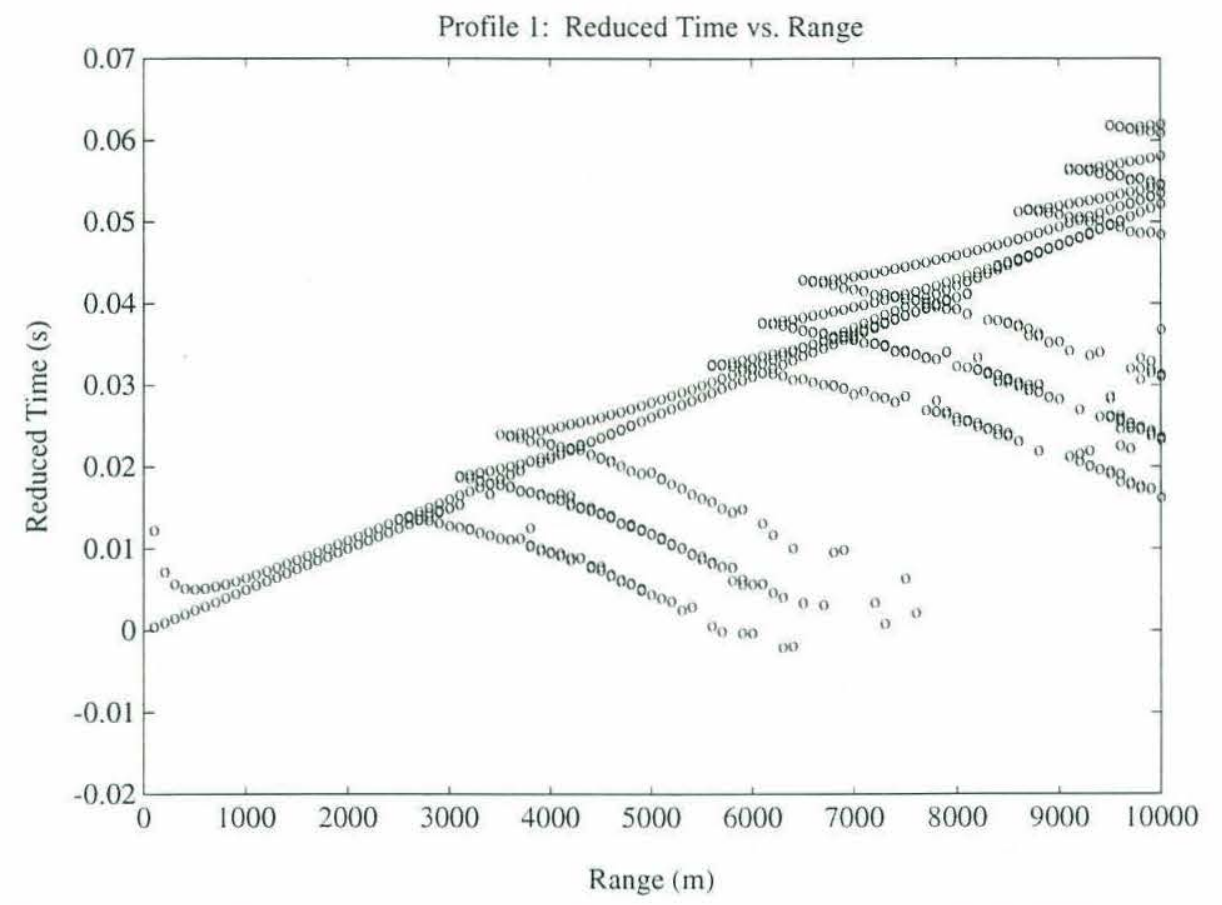

Figure 2.18: Reduced time vs. range, for source and receiver at $30 \mathrm{~m}$ depth.

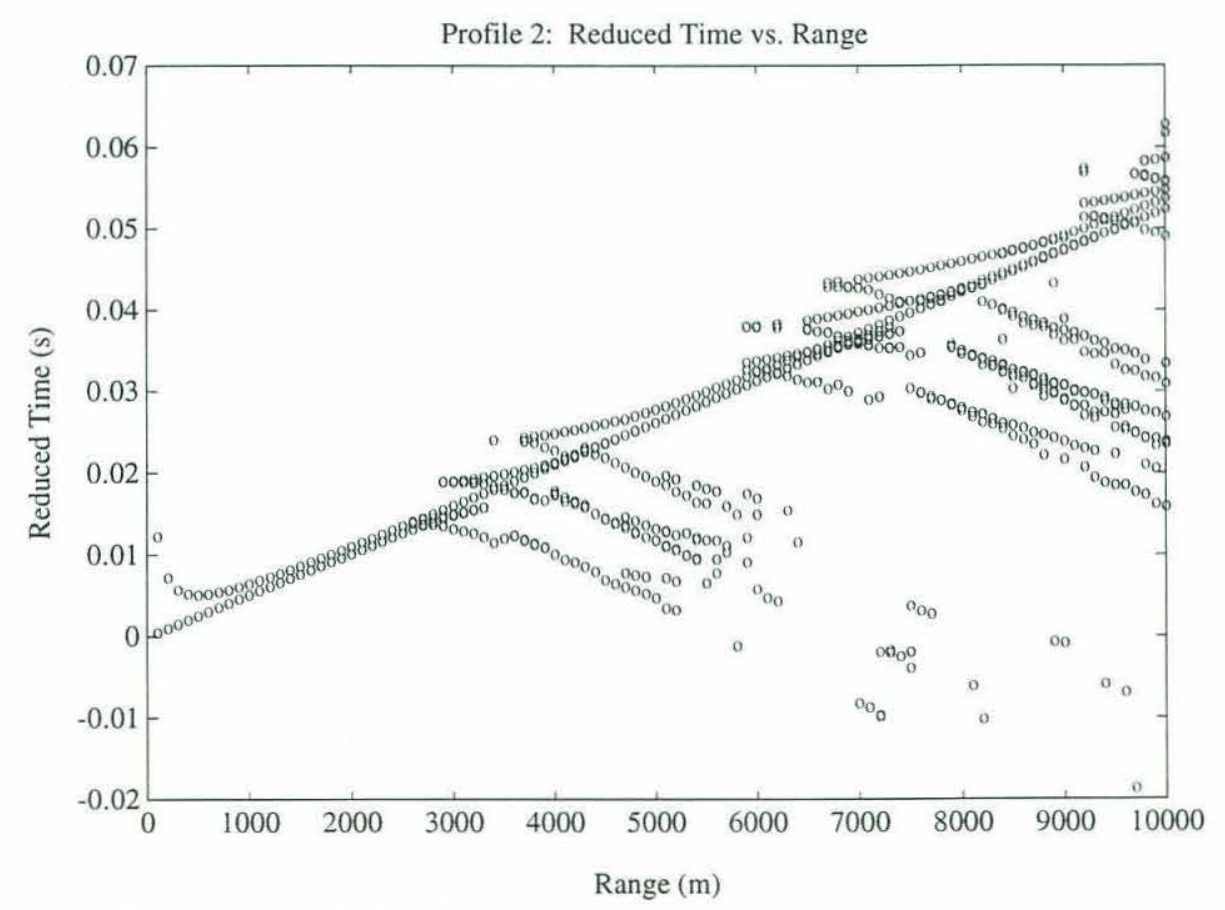

Figure 2.19: Reduced time vs. range 24 hours later 
VARIATION OF ANGLE WITH RANGE

Figure 2.20 and 2.21 show the variation of arrival angle with range. As in the case of time, over most of the plot, angle is changing linearly with range. The edges of the linear segments, however, are characterized by large slopes rather than by discontinuities as with travel time. Figure 2.20 was generated using the same sound speed profile as Figure 2.18, and Figure 2.21 was generated using the same 24-hour older profile as in Figure 2.19 .

While the range vs. time relationship held great promise for the possibility of a locally linearized inversion from travel time data, the large derivatives at the transitions between linear segments in the angle data could cause problems. Combined with a likely angle measurement noise of about one degree, however, the variation in angle is not very sensitive to range changes. This means that little information would be lost by arbitrarily setting the derivatives of angle with respect to range to zero. The other side of this issue is that the relative immunity of angle to range changes makes angle an extremely useful measurement for path classification.

\section{VARIATION OF TRAVEL TIME WITH EOF WEIGHT}

In Figure 2.22, the small circles indicate the travel times evaluated for integer weights on the first EOF from -20 to 20 at a range of $3 \mathrm{~km}$. Source and receiver depths are $30 \mathrm{~m}$. The lines between circles show the direction of the derivative calculated at each point. The travel time variation with sound speed profile parameter is predictable by a linear approximation as long as the ray path being observed does not fade. Figure 2.23 presents the same data as Figure 2.22, except that the range is $10 \mathrm{~km}$. At both ranges, the variation of travel time with sound speed profile parameter is predictable by the first derivative as long as the path does not fade. 
Profile 1: Arrival Angle vs. Range

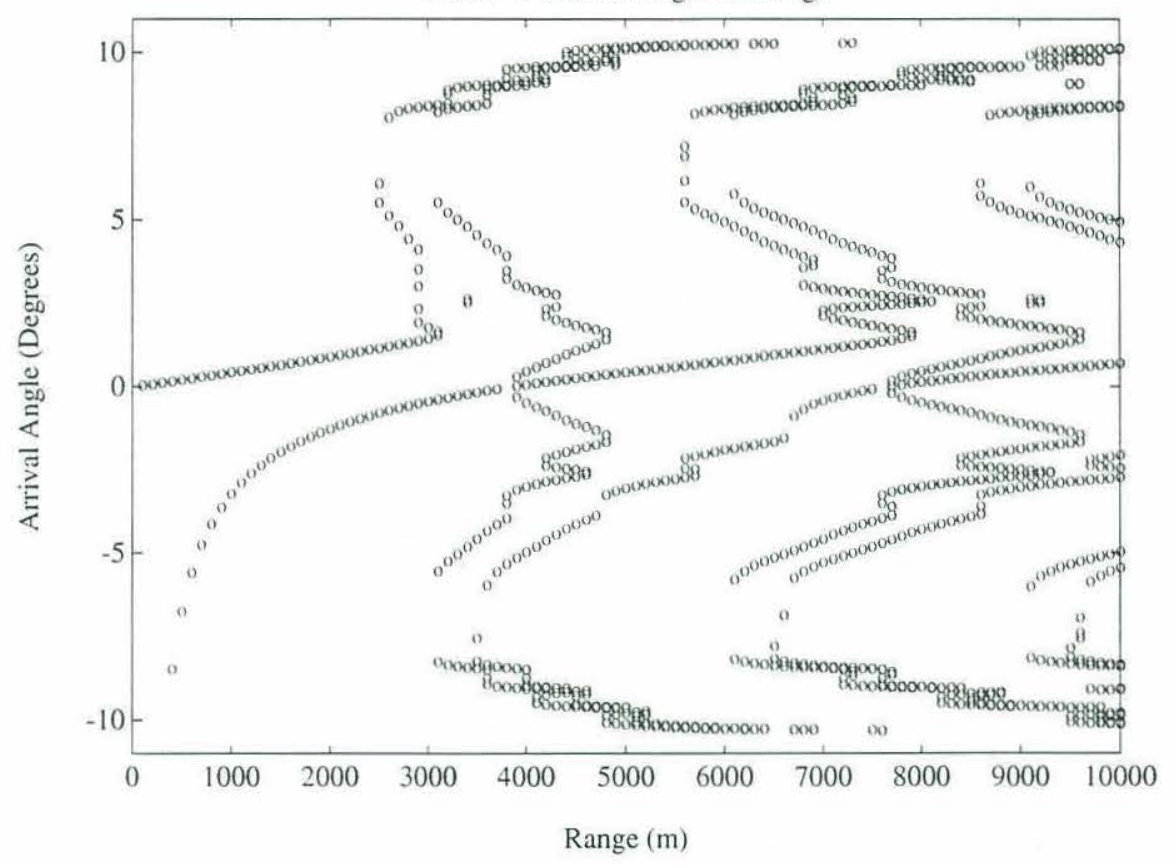

Figure 2.20: Arrival Angle vs. Range. Source and receiver depths are 30m.

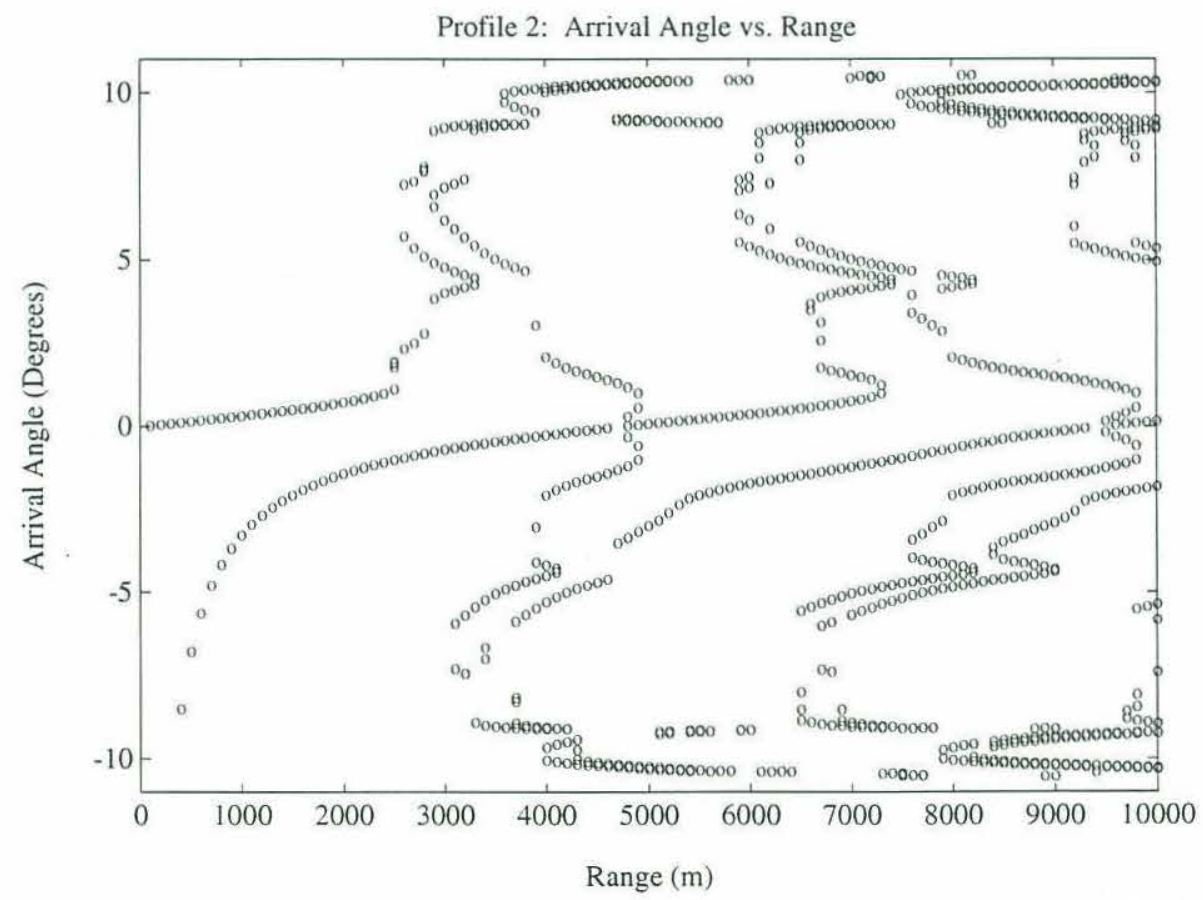

Figure 2.21: Arrival Angle vs. Range 24 hours later. Source and receiver depths are $30 \mathrm{~m}$. 
Travel Time vs. Profile Parameter $(3 \mathrm{~km})$

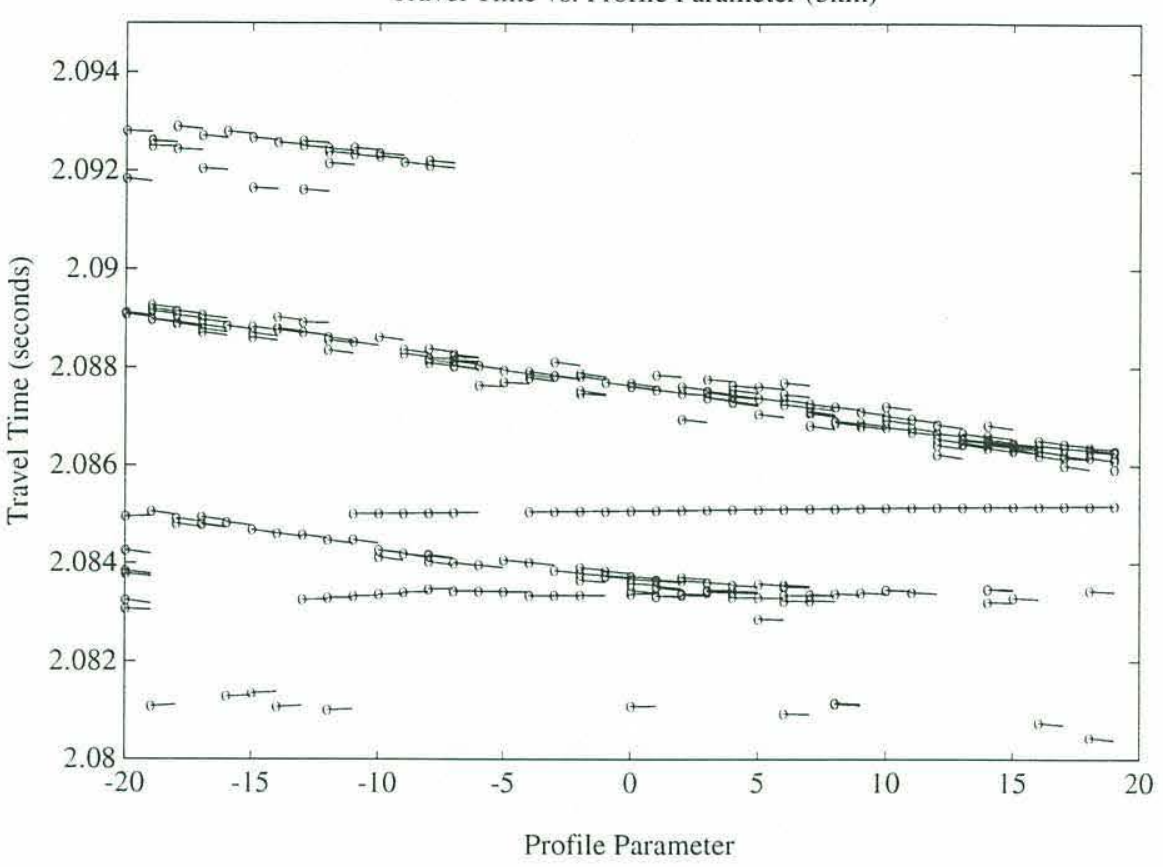

Figure 2.22: Travel time vs. the weight of the first EOF at $3 \mathrm{~km}$

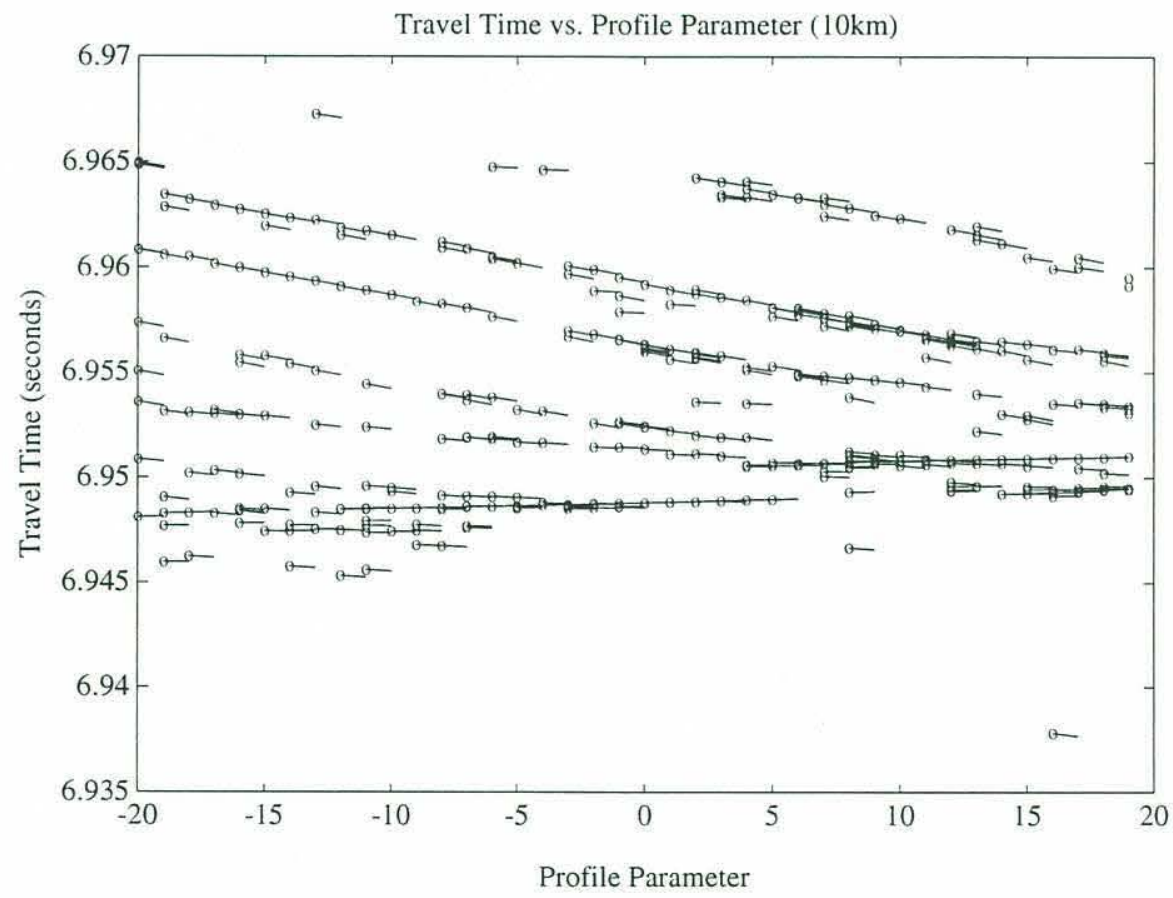

Figure 2.23: Travel time vs. the weight of the first EOF at $10 \mathrm{~km}$ 


\section{VARIATION OF ARRIVAL ANGLE WITH PROFILE PARAMETER}

Figure 2.24 and 2.25 show the variation of arrival angle with the weight of the first

EOF. A local linearization should be valid over most of the parameter region. There are a few dramatic exceptions, however, where huge derivatives are produced. The plot is given for both $3 \mathrm{~km}$ and $10 \mathrm{~km}$ ranges. As in the case of time, over most of the plot angle is changing linearly with profile parameter. The edges of the linear segments, however, are characterized by large slopes rather than by discontinuities as with travel time.

The huge spikes in derivative which do not represent a long-term trend in arrival position mean that using the derivatives of arrival angle for a linearized inversion can produce inaccuracies at certain locations. Angle measurement accuracy is likely about one degree. This means that arrival angle contributes little information to the inversion for EOF weights. On the other hand, the fact that it changes so little with EOF weight makes arrival angle quite useful for path identification. This result is similar to that observed for the variation of arrival angle with range.

Because of the small effect of range and EOF weights on arrival angle, and because arrival angles occasionally manifest large derivatives that are not indicative of longer term trends, arrival angles will not be used the linear inversion. Because of their relative insensitivity to the parameter space, arrival angles are quite useful for arrival matching. For this purpose their derivatives can be approximated as being zero with respect to both range and EOF weights. 


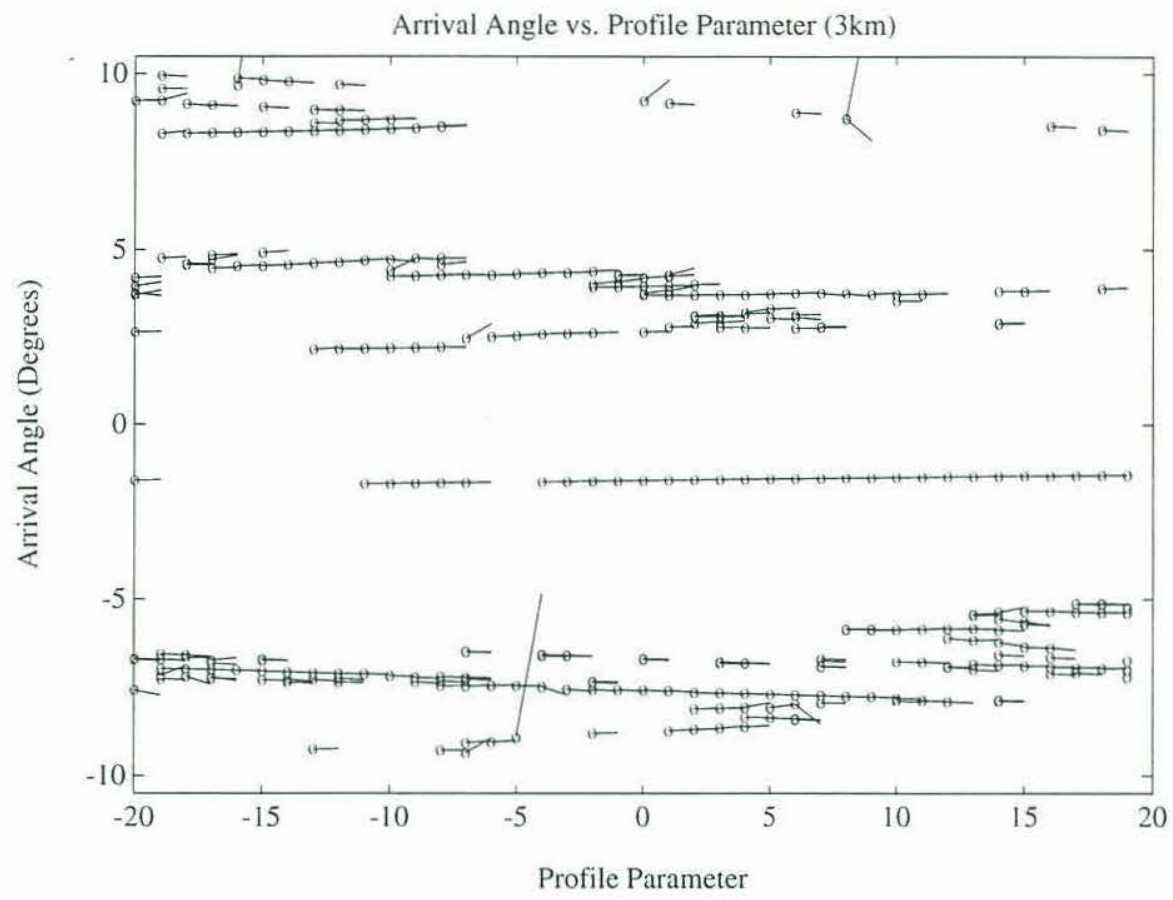

Figure 2.24: Arrival angle vs. the weight of the first EOF at $3 \mathrm{~km}$

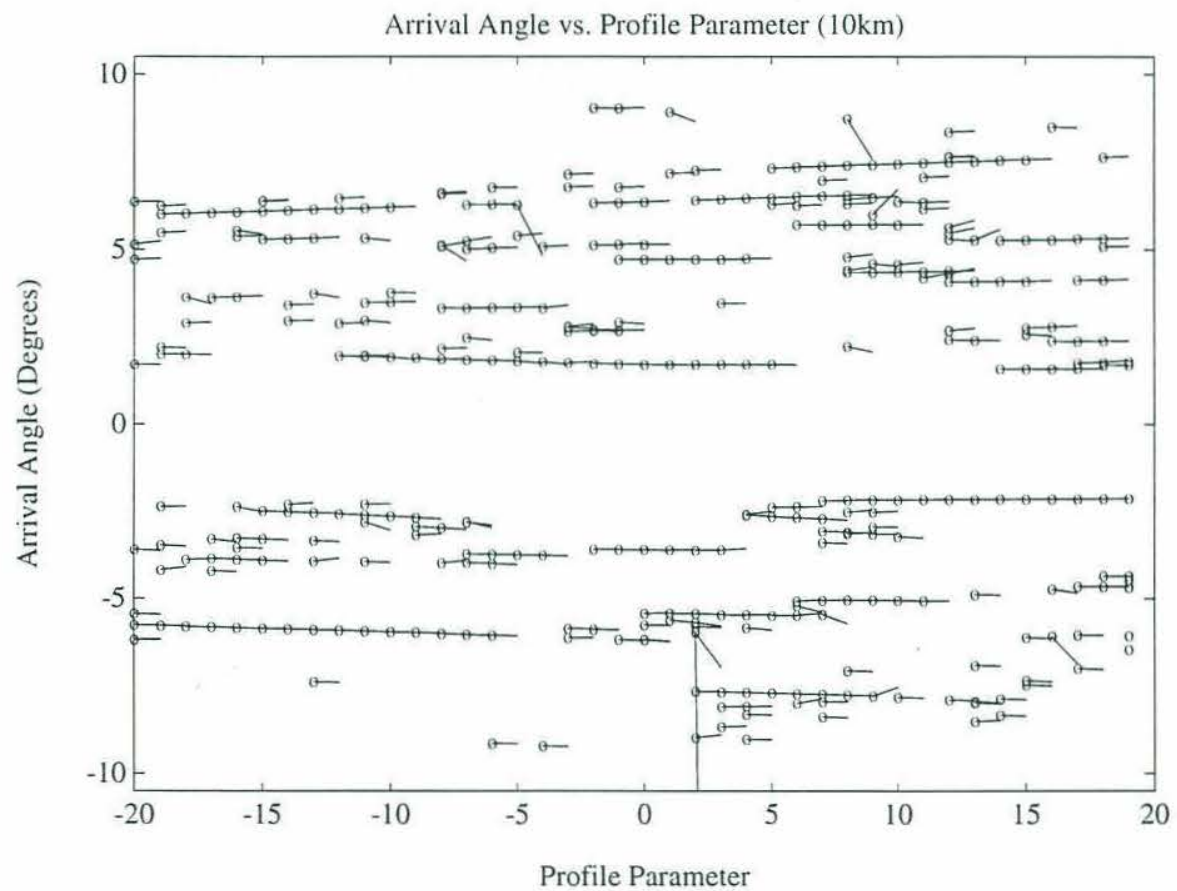

Figure 2.25: Arrival Angle vs. the weight of the first EOF at $10 \mathrm{~km}$ 


\subsection{REVISED SYSTEM STRUCTURE}

Over the scale of parameter estimate errors, as established by Section 2.5, arrival times tend to behave linearly with change in range and EOF weight as long as their corresponding ray path does not fade. Thus, if a set of eigenrays can be found which do not fade over the region of parameter uncertainty, then a linear inversion can be made for the system parameters. This observation forms the basis for the division of the navigation problem into two parts, a path matching and a linear inversion.

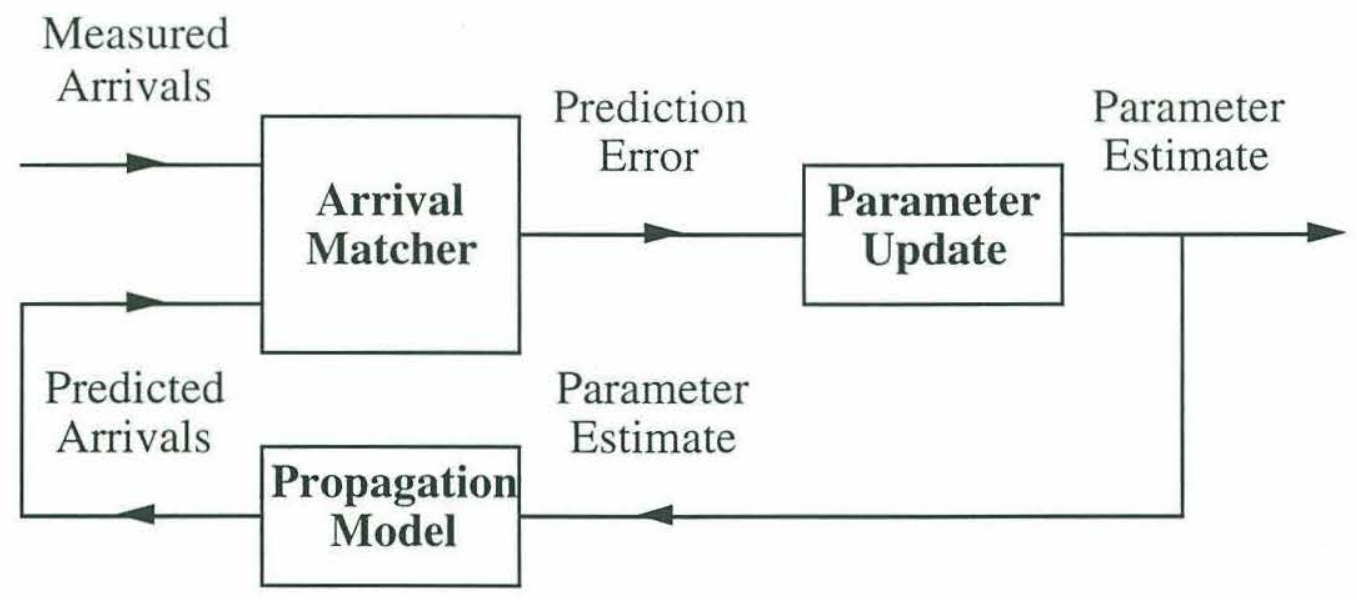

Figure 2.26: Multipath Matching Navigation System

The arrival matcher seeks to determine which measured arrival corresponds to each predicted arrival. This allows calculation of a prediction error as the difference between predicted and measured travel times. Based on a linearization about the current parameter estimate, these prediction errors can be inverted to determine the parameter estimate errors, enabling an improved parameter estimate to be found.

This multipath matching navigation system is subject to global estimation errors, which correspond to failure of the arrival matcher, and to local estimation errors, which correspond to the performance of the parameter update given a correct arrival matching. 
The arrival matcher will be the focus of Chapter 3, and the parameter inversion will be the topic of Chapter 4. 


\section{Chapter 3 Arrival Matching}

\subsection{INTRODUCTION}

At the end of the last chapter, a revised navigation system structure was proposed which divided the navigation problem into two components: an arrival matcher, which identified each measured arrival with a particular ray path through the water, and a linear parameter estimator. Global estimation errors come from a failure of the eigenray matcher to place the variation of a particular measured arrival in the correct linear regime, i.e. failure to identify it with the correct ray path. This chapter will focus on the business of avoiding global errors, that is, on achieving an optimal match between the measured and predicted arrivals. First, a simple least distance matching algorithm is used to match the arrivals. However, the differences between predicted and measured arrivals are highly correlated, since all the rays sample the same environment. The least distance matching throws away the information in these correlations. A new matching criteria is then defined to account for correlations between arrival shifts. A fast matching algorithm is described to speed this matching. Finally a comparison is drawn between the ultimate parameter estimate accuracies resulting from least distance and optimal matching. 


\subsection{LEAST DISTANCE MATCHING}

If the shifts in the arrival locations are uncorrelated from one ray path to the next, then a least distance matching algorithm is appropriate, and one of many linear programming methods to solve assignment problems can be applied. A cost matrix is defined where the columns of the matrix correspond to the $M$ measured arrivals, and the rows correspond to the $P$ predicted arrivals. The elements of the matrix are then

$$
\mathrm{C}_{i j}=\text { cost of matching ith prediction to jth measurement }
$$

The possibility that some arrivals may not have matches is handled by the assignment of "dummy" rows and columns [Ignizio, 1982]. A fixed penalty $\alpha$ will be assigned to leaving an arrival unmatched. To incorporate this, $M-1$ "unmatched measurement" rows are added to the $P$ prediction rows so that as many as $M$ - 1 of the $M$ measured arrivals may remain unmatched if necessary. This requires that at least one measured arrival be matched. The cost penalty for these unmatched measurements is set to the value $\alpha$. Similarly, $M$ the measurement columns are extended with $P$ - 1 "unmatched prediction" columns, so that as many as $P-1$ of the $P$ predicted arrivals may remain unmatched if necessary. This requires that at least one predicted arrival be matched. The cost penalty for these unmatched predictions is also $\alpha$. If more than one matching can be made between the predicted and measured arrivals, the unmatched rows and columns will not all have to be used. For computational efficiency, these should be kept out of circulation in the matching problem until they are needed. A small constant $\varepsilon$ is subtracted from the penalty for "matching" the jth unmatched column with the jth unmatched row, this ensures that the algorithm will not waste time seeking a best match between the unmatched 
rows and columns. The cost matrix constructed in this way is shown below. The bold elements are the row and column labels. In the example below, $\mathrm{P}<\mathrm{M}$.

$\begin{array}{ccccccccc} & m_{1} & m_{2} & \ldots & m_{M} & u_{1} & u_{2} & \ldots & u_{P-1} \\ p_{1} & C_{11} & C_{12} & \ldots & C_{1 M} & \alpha & \alpha & \ldots & \alpha \\ p_{2} & C_{21} & C_{22} & \ldots & C_{2 M} & \alpha & \alpha & \ldots & \alpha \\ : & : & : & & : & : & : & & : \\ p_{P} & C_{\mathrm{P} 1} & C_{\mathrm{P} 2} & \ldots & C_{P M} & \alpha & \alpha & \ldots & \alpha \\ \boldsymbol{u}_{1} & \alpha & \alpha & \ldots & \alpha & \alpha-\varepsilon & \alpha & \ldots & \alpha \\ \boldsymbol{u}_{2} & \alpha & \alpha & \ldots & \alpha & \alpha & \alpha-\varepsilon & \ldots & \alpha \\ : & : & : & & : & : & : & & : \\ \boldsymbol{u}_{\boldsymbol{P}-1} & \alpha & \alpha & \ldots & \alpha & \alpha & \alpha & \ldots & \alpha-\varepsilon \\ \boldsymbol{u}_{\boldsymbol{P}} & \alpha & \alpha & \ldots & \alpha & \alpha & \alpha & \ldots & \alpha \\ : & : & : & & : & : & : & & : \\ \boldsymbol{u}_{\mathrm{M}-1} & \alpha & \alpha & \ldots & \alpha & \alpha & \alpha & \ldots & \alpha\end{array}$

Mack's method is used to solve the assignment problem [Bunday, 1984]. 


\subsection{OPTIMAL MATCHING}

The least distance matching algorithm implicitly assumed uncorrelated measurable quantity errors by assuming that the optimal match for one arrival was independent of the optimal match for another arrival. However, the variations in the measurable quantities for various arrivals are correlated, and the calculation of derivatives relating parameter changes to measurable quantity changes enables a calculation of these correlations given a statistical characterization of parameter variations. In this section, knowledge of the correlations between measurable quantity prediction errors is exploited to improve matching performance. Let $\mathbf{y}$ be the vector of measurements (before matching) and $\hat{\mathbf{y}}$ the vector of predictions. Define a match vector $\mathbf{m}$ where $\mathbf{m}_{\mathrm{i}}=\mathrm{j}$ indicates that $\hat{\mathbf{y}}_{\mathrm{i}}$ is to be matched with $\mathbf{y}_{\mathbf{j}}$. The match vector is sought which has the greatest conditional probability given the measurements $\mathbf{y}$.

$$
\mathbf{m}_{B}=\max _{\mathbf{m}} p(\mathbf{m} \mid \mathbf{y})
$$

By Bayes' Rule:

$$
p(\mathbf{m} \mid \mathbf{y})=\frac{p(\mathbf{y} \mid \mathbf{m}) p(\mathbf{m})}{p(\mathbf{y})}
$$

Recognizing that $\mathrm{p}(\mathbf{y})$ is independent of the matching, the maximization can be rewritten:

$$
\mathbf{m}_{B}=\max _{\mathbf{m}} p(\mathbf{y} \mid \mathbf{m}) p(\mathbf{m})
$$


Errors are taken to be Gaussian, and $p(\mathbf{y} \mid \mathbf{m})$ can be calculated. For this calculation, define

$\mathbf{y}_{\mathbf{m}}$ as the vector of measured arrivals where $\mathbf{y}_{\mathbf{m i}}$ is matched to $\hat{\mathbf{y}}_{\mathrm{i}}$. The error covariance matrix of the parameters is $\mathrm{Q}$, and the covariance of the measurement noise in $\mathbf{y}_{\mathbf{m}}$ is $\mathrm{R}$. The measurement errors are related to the parameter errors by the linear approximation $\mathbf{y}_{\mathbf{m}}$ $=\mathrm{Cx}+\mathbf{n}$.

$$
\begin{gathered}
p(\mathbf{y} \mid \mathbf{m})=\frac{1}{(2 \pi)^{N / 2}|\mathrm{P}|^{1 / 2}} \exp \left[-\frac{1}{2}\left(\left(\mathbf{y}_{\mathbf{m}}-\hat{\mathbf{y}}\right)^{\mathrm{T}} \mathrm{P}^{-1}\left(\mathbf{y}_{\mathbf{m}}-\hat{\mathbf{y}}\right)\right)\right] \\
\mathrm{P}=\mathrm{C} \mathrm{Q} \mathrm{C}^{\mathrm{T}}+\mathrm{R}
\end{gathered}
$$

At most one element from $\mathbf{y}$ is matched with each element of $\hat{\mathbf{y}}$. All the elements of $\hat{\mathbf{y}}$ need not be matched. There may be some eigenrays $\hat{\mathbf{y}}$, call them $\hat{\mathbf{y}}_{\mathbf{n}}$, for which no suitable match appears in $\mathbf{y}_{\mathbf{m}}$. The problem remains of what values to assign to the "missing" elements of $\mathbf{y}_{\mathbf{m}}$--the values which are intended to match $\hat{\mathbf{y}}_{\mathbf{n}}$. Call these missing elements $\mathbf{y}_{\mathbf{m} \mathbf{n}}$, and call the elements of $\mathbf{y}_{\mathbf{m}}$ for which an assignment from $\mathbf{y}$ has been made $\mathbf{y}_{\mathbf{m a}}$. The missing values $\mathbf{y}_{\mathbf{m n}}$ can then be filled in with their most probable values based on the assigned paths:

$$
\mathbf{y}_{\mathbf{m n}}=\underset{\mathbf{y}_{\mathbf{m n}}}{\operatorname{argmax}} \mathrm{p}\left(\mathbf{y}_{\mathbf{m n}} \mid \mathbf{y}_{\mathbf{m a}}\right)
$$

Unmatched arrivals are assigned their estimated value, so some penalty must be exacted elsewhere for not matching an arrival, otherwise the optimum match would be all predicted arrivals unmatched. This penalty is taken in the value of $p(\mathbf{m})$,

$$
p(\mathbf{m})=c \alpha^{n}
$$


where $\alpha$ is a penalty factor for each unmatched arrival, $\alpha<1$, and $n$ is the number of unmatched arrivals in $\mathbf{m}$. In general it is much worse to mismatch an arrival than to fail to recognize a correct match, since a mismatch can cause havoc in the parameter estimate. The penalty for not matching a prediction should be small, otherwise the matching algorithm may be forced to make rather dubious matches to avoid the penalty. The constant $c$ is a scaling factor to make the probabilities of all possible matches sum to one, so that $\mathrm{p}(\mathbf{m})$ is a legitimate probability mass function. This scaling factor $c$ can be ignored in the actual implementation because removing it will not effect the location of the maximum which is sought.

Equation 3.2.3 in combination with 3.2.4, 3.2.5, and 3.2.6 provides a measure of "goodness of match" which takes into account the correlations between errors in predicted values. To evaluate this function for every possible match would be time consuming. For a system of only one beacon, if there are $N$ predicted and $M$ measured arrivals, the number of possible match vectors is:

$$
\sum_{n=0}^{\min (N, M)} \frac{M !}{(M-n) !}\left(\begin{array}{c}
N \\
N-n
\end{array}\right)
$$

The summation is over the number of predictions which will be matched out of the total of $N$ predictions. The maximum number of matches possible is the lower of $N$ and $M$. There are $n$ predictions which must be matched from the $M$ possible measurements, giving the $M$ ! / (M-n)! term. This leaves $N$-n unmatched predictions. There are $\left(\begin{array}{c}N \\ N-n\end{array}\right)$ ways to choose which $N-n$ predictions to leave unmatched. The table below gives some examples of this summation: 


$\begin{array}{ccc}\text { Predictions } & \text { Measurements } & \text { Matches } \\ 1 & 1 & 2 \\ 2 & 2 & 7 \\ 3 & 3 & 34 \\ 5 & 5 & 1546 \\ 7 & 7 & 130922 \\ 9 & 9 & 17572114\end{array}$

This table shows the number of possible matches for a single beacon. Navigation requires at least three beacons. A predicted arrival from one beacon should never be matched to a measured arrival from another beacon; however, the objective function for each beacon will depend on all the matches for all the beacons. The errors in measurable quantities between beacons are coupled through their common dependence on the parameter set. If a function $m(N, M)$ equal to equation 3.2.7 is defined, then the total number of matches which must be evaluated for $\mathrm{B}$ beacons is:

$$
\prod_{b=1}^{B} \mathrm{~m}\left(\mathrm{~N}_{\mathrm{b}}, \mathrm{M}_{\mathrm{b}}\right)
$$

For a typical three-beacon system with 5 arrivals from one beacon, 7 from another and 3 from a third, this means $6,881,784,008$ matches which must be evaluated. Clearly this is impossible unless the number of matches that must be checked can be reduced. 


\subsection{FAST MATCHING ALGORITHM}

The matching method defines a process to obtain a Bayesian estimate of the correct match between predicted and measured arrivals. To evaluate the objective function for each possible match would be prohibitively time consuming for even a modest number of eigenrays from a modest number of beacons. A fast matching algorithm can be created by exploiting the fact that it is usually fairly obvious what most of the matches should be, and for the matches which are not clear, the ambiguity in matching regards only a few possibilities, not the entire measurement set. The fast matching algorithm restricts the number of matches which must be checked by searching only those matches which are not obviously wrong.

In the fast matching algorithm, a reasonable match is made by some simpler (but non-optimal) matching rule. The objective function for this reasonable match is evaluated. This serves as a lower bound on the objective function of the optimal match. With this lower bound in mind, the search of the matching space begins. All possible matches (and the possibility of no match) for the first predicted arrival are tried. For each match of the first predictions, the remaining elements in the matched measurement vector $\mathbf{y}_{\mathbf{m}}$ are assigned their most probable value. This objective function for this match is evaluated, and its value is an upper bound on all matches which have the same first prediction matches. If this upper bound does not exceed the lower bound generated by the reasonable match, then no matches which contain the current first arrival match need to be checked. They will not improve upon the reasonable match bound. The first matches which obtained an upper bound that was greater than the lower bound imposed by the reasonable match are retained

in the feasible set. Next, the second predicted arrivals are matched, and the process is repeated, finding an upper bound on all matchings with the current "prefix", and excluding 
all "prefixes" whose upper bound does not exceed the reasonable match lower bound. This

process of growth by one layer and then trimming of the feasible match tree is diagrammed below.
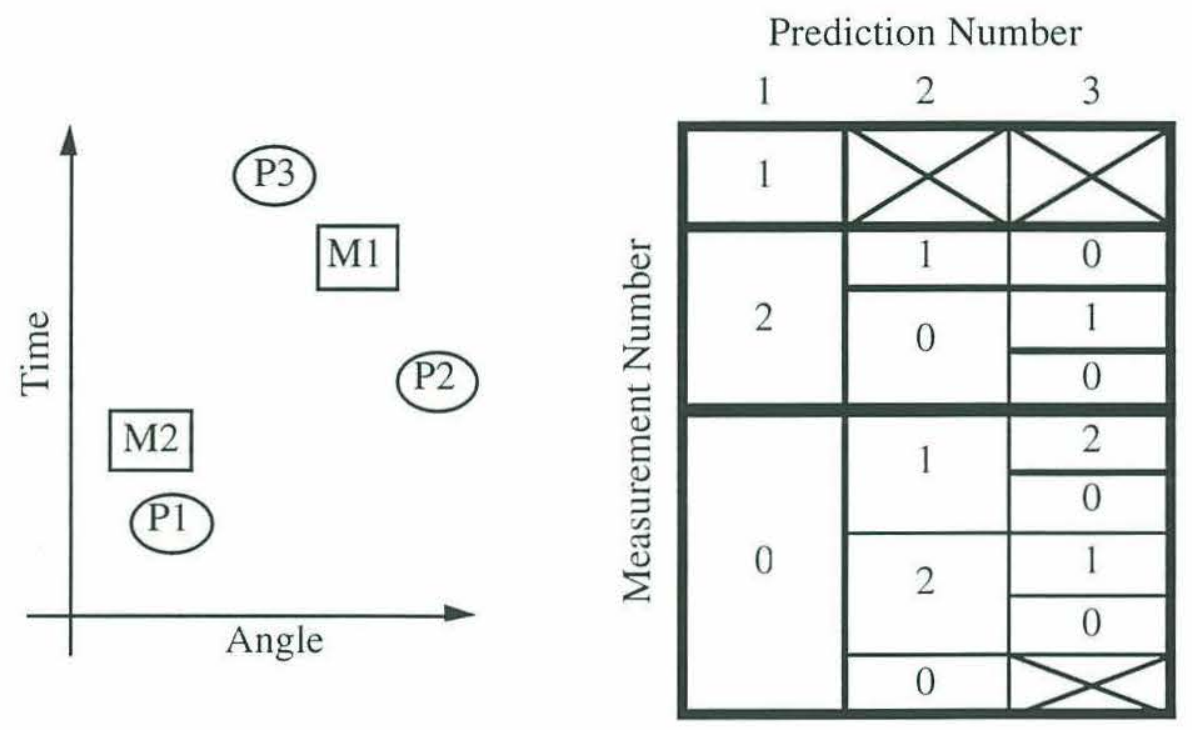

Figure 3.1: Fast Matching Algorithm

In figure 3.3.1 on the left, the predicted and measured arrivals are shown as points in time-angle space. The predicted arrivals are circled, and the measured arrivals are boxed. A least distance matching algorithm matched prediction 1 to measurement 2 and prediction 3 to measurement 1 . Prediction 2 is left unmatched. The objective function evaluated for this match will serve as the lower bound. The fast matching algorithm begins by trying all possible assignments for the first prediction. This is represented by the first column in the box in figure 3.3.1. The numbers in the cells in the first column are the number of the measurement to which the first prediction is matched. The zero indicates leaving the prediction unmatched. In the top cell, the first prediction is matched with the first measurement, and the objective function is already too low, so no matches where the first prediction is matched to the first measurement need to be considered. The other matching possibilities could not be excluded after the first match. Matching proceeds to the 
second prediction, and the feasible match prefixes are retained, while the infeasible ones are trimmed. Once all the predictions have had matches assigned, the match with the highest probability among the feasible matches is retained. 


\subsection{IMPORTANCE OF CORRELATIONS}

The various eigenrays which connect the source and receiver all sample the medium and are therefore effected by the same parameter set. This means changes in arrival angles and travel times should be correlated due to their common dependence on the parameter set. Below, the errors in parameter estimates using the rays matched by the least distance matcher and by the optimal matcher are compared.

The expression for the covariance of the measurable quantities $\mathbf{t}$ gives us insight into this issue of how important correlations might be for matching.

$$
\operatorname{Cov}(\mathbf{t})=\mathrm{CP} \mathrm{C}^{T}+\mathrm{R}
$$

There are two sources of uncertainty in the value of $\mathbf{t}$. There is uncertainty in the parameters themselves, viewed through the matrix $\mathrm{C}$, and there is the measurement error, which is a catch-all term for both errors in measuring the arrival time, and for variations in $\mathbf{t}$ from causes that are not modeled. It is assumed that $\mathrm{R}$ is dominated by the uncorrelated measurement noise.

The least distance matcher and the optimal matcher are equivalent when the covariance matrix is close to diagonal. This can happen for small parameter errors, where $\mathrm{R}$ dominates equation 3.4.1, and it can happen if the eigenrays involved happen to sample the environment in such a way as to make the covariance close to diagonal.

In the graph below, the least distance matcher (solid line) and the optimal matcher (dashed line) are compared. The true beacon range is $3 \mathrm{~km}$. The initial range estimate error is on the x-axis. The measured and predicted arrivals are generated, and both routines 
choose their matching. Based on the two matchings, two updated range estimates are generated, and their errors are shown.

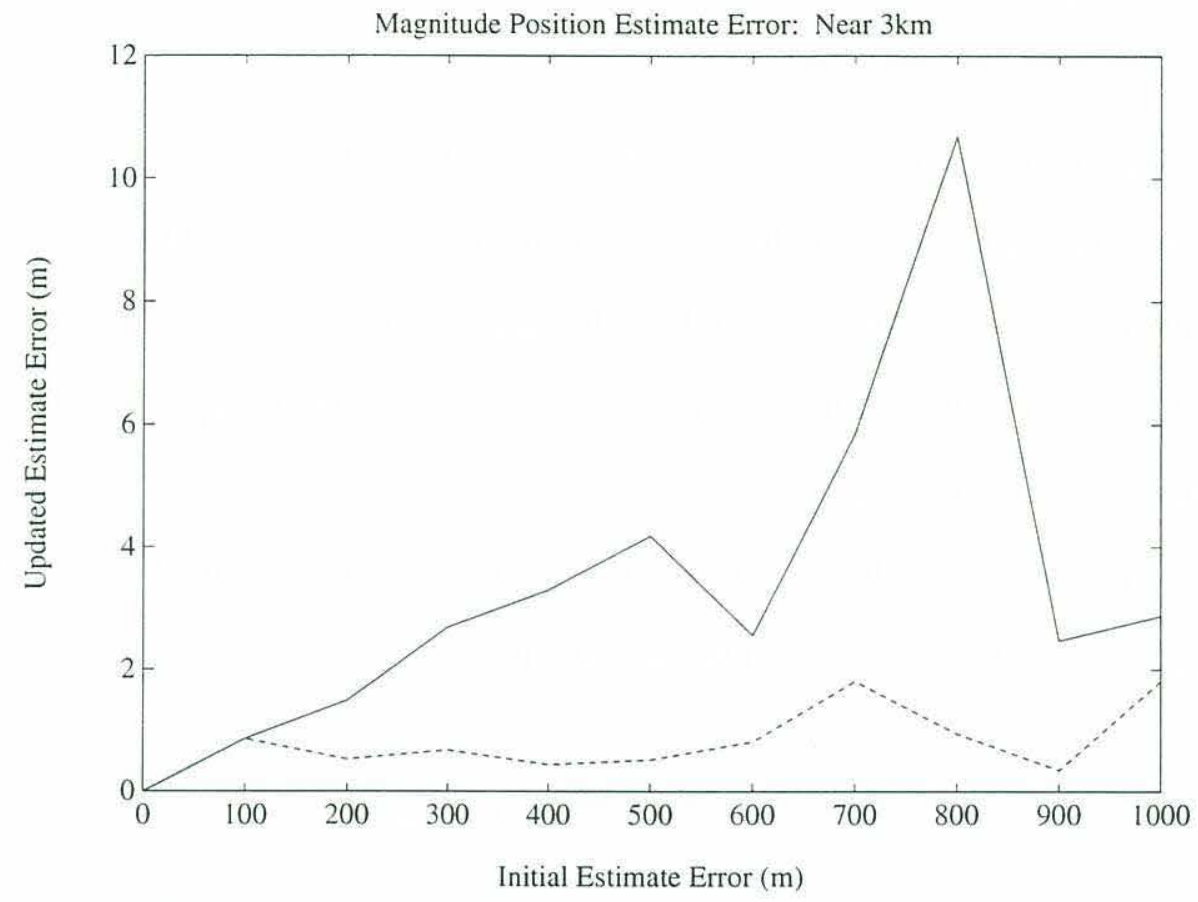

Figure 3.2: Updated range estimate error for both matching routines at $3 \mathrm{~km}$

The experiment above is repeated for $10 \mathrm{~km}$ range.

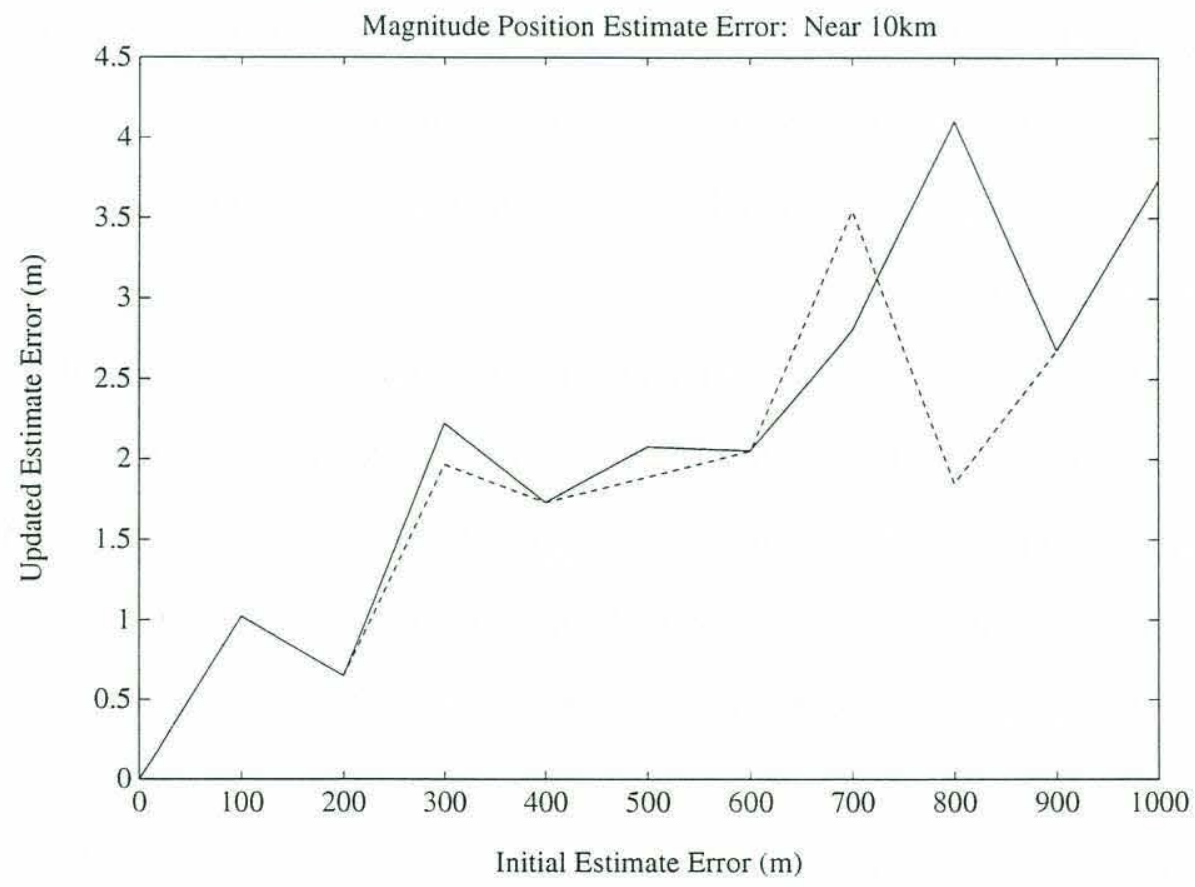

Figure 3.3: Updated range estimate error for both matching routines at $10 \mathrm{~km}$. 
For the range estimation problem, the range estimate accuracy is a little better for the optimal matching routine, but both routines performed so well due to the linearity of the range-time relationship that the differences are probably unimportant in practical situations.

Next, the above experiment was repeated, but this time range was held constant, and the initial EOF weight estimate for the first EOF was changed. This experiment was carried out for the $3 \mathrm{~km}$ and $10 \mathrm{~km}$ ranges.

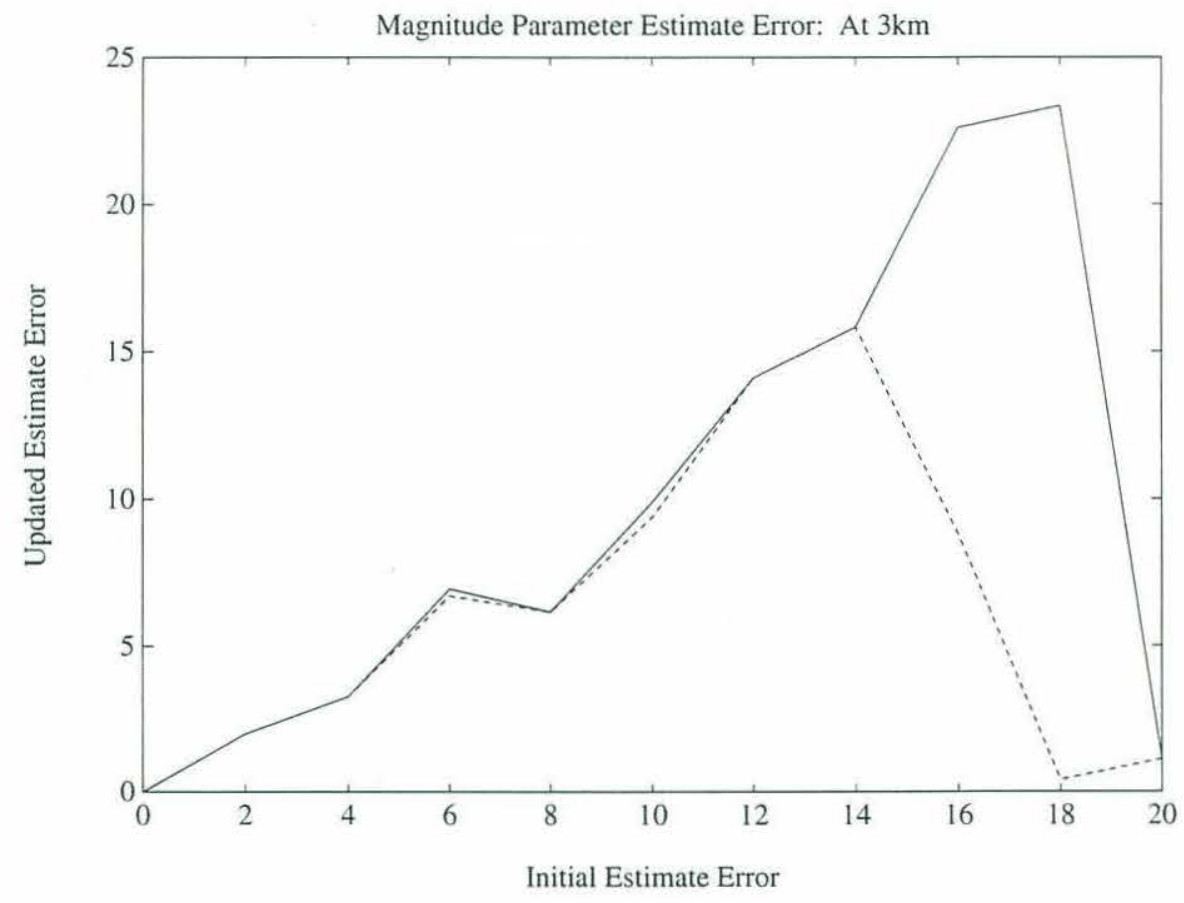

Figure 3.4: Updated EOF weight estimate error for both matching routines at $10 \mathrm{~km}$.

The estimation accuracy at this three kilometer range is very poor for both estimators. Such poor accuracy occurs when the eigenrays do not significantly sample the depths where the EOFs are large-valued. This poor accuracy at short ranges recalls the poorer accuracy inside the beacon array observed in the Cramér-Rao bound for profile parameter estimation in chapter 2. Next the experiment is repeated for a range of $10 \mathrm{~km}$. Accuracy is much improved, because there is a greater diversity of ray paths, and the paths tend to dive deeper, into the depths where the basis function is largest. 


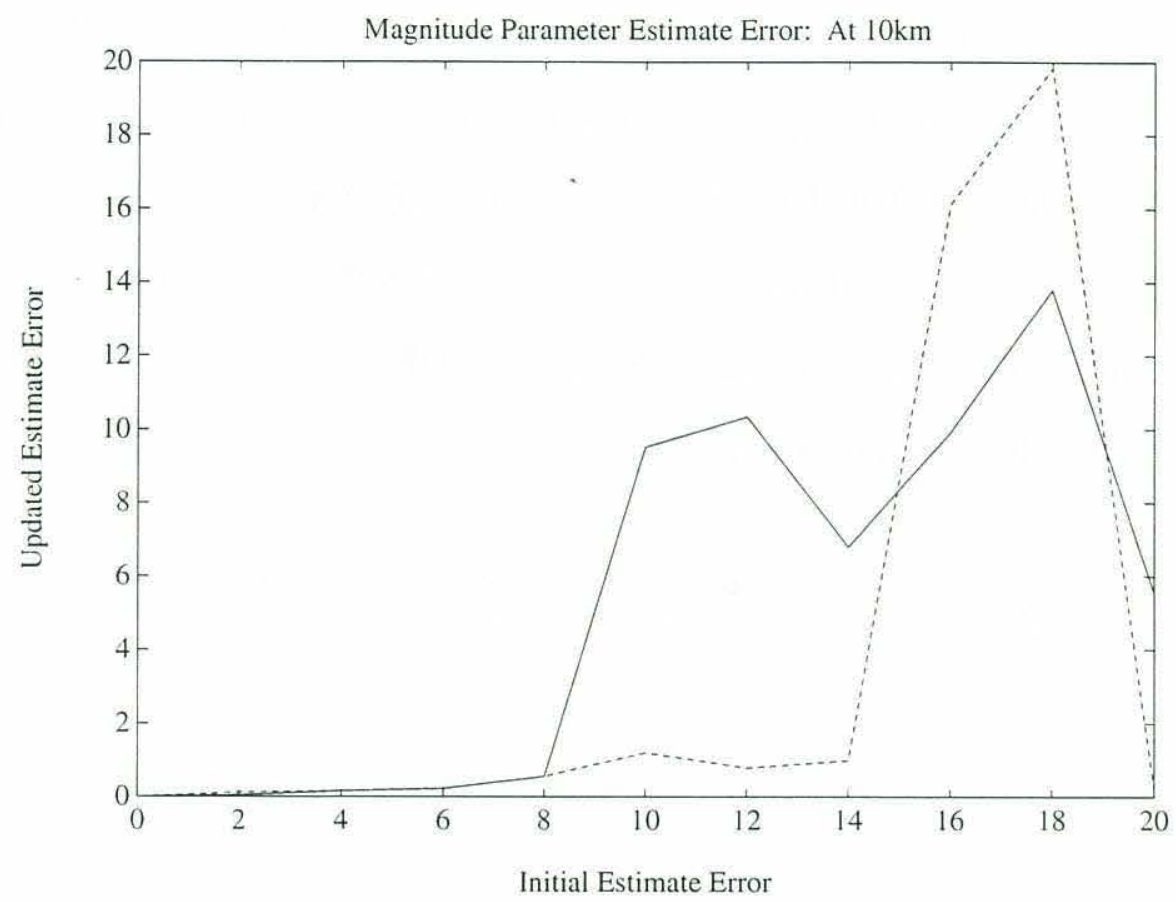

Figure 3.5: Updated EOF weight estimate error for both matching routines at $10 \mathrm{~km}$.

In the $10 \mathrm{~km}$ case, the performance for both estimators was good at small parameter errors. At an intermediate range, the optimal estimator yields a substantially better estimate. Presumably the least distance matcher begins misidentifying rays. At large errors, both estimators have difficulty. 


\section{Chapter 4 Parameter Estimation}

\subsection{INTRODUCTION}

In the previous chapter, global estimation errors, i.e. mismatched arrivals, were discussed. In this chapter, it will be assumed that the correct lobe on the energy function has been found, and the ability to find its maximum using noisy measurements will be explored. A sense of the size and shape of the local estimation errors can be had from the Cramér-Rao bounds discussed in Chapter 2. Whereas in Chapter 2 perfect knowledge of either sound speed or position was assumed, here all parameters will be treated as random variables. Based on the covariances of the parameters and of the measurement noise, the covariance of the parameter estimate is found for position, time synchronization, and the weights on the three EOFs which represent the sound speed profile. 


\subsection{LINEAR ESTIMATES}

An extended Kalman filter is used to invert for vehicle $\mathrm{x}$ - and $\mathrm{y}$-position, vehicle clock drift, and weights for the three EOFs. The system is linearized around the best parameter estimate $\mathbf{x}_{\mathbf{0}}$. The variation in parameter value from the linearization point is $\Delta \mathbf{x}$, and the corresponding variation in measurable quantity is $\Delta \mathbf{y}$. The unpredictable portion of the change in parameter at each time step is modeled as a Gaussian noise vector $\mathbf{w}(\mathrm{t})$ with covariance matrix $\mathrm{P}$. The measurement noise $\mathbf{n}(\mathrm{t})$ has covariance $\mathrm{R}$.

$$
\begin{gathered}
\Delta \mathbf{x}(\mathrm{t}+1)=\mathbf{w}(\mathrm{t}) \\
\Delta \mathbf{y}(\mathrm{t})=\mathrm{C}\left(\mathbf{x}_{\mathbf{0}}\right) \Delta \mathbf{x}(\mathrm{t})+\mathbf{n}(\mathrm{t})
\end{gathered}
$$

The matrix $\mathrm{C}$ represents a particular linearization about a parameter value $\mathbf{x}_{\mathbf{0}}$. The regimes of validity about $\mathbf{x}_{\mathbf{0}}$ of a particular linearization $\mathrm{C}\left(\mathbf{x}_{\mathbf{0}}\right)$ can be roughly estimated from the figures in section 2.6 to be on the order of a kilometer in range and a sizable portion of the region of sound speed profile parameter variation. For a vehicle moving at 2 $\mathrm{m} / \mathrm{s}$ and beacons transmitting every 10 seconds, on the order of 50 sets of received signals will be acquired with approximately the same linearization in effect. It might be supposed from this that the limiting case of the parameter estimate covariance matrix, often referred to as $\mathrm{P}_{\infty}$, would be reached.

The measurement noise term $\mathbf{n}(\mathrm{t})$ is a catch-all term including "true" measurement noise which can be considered to be independent from one observation to the next and also any features of the environment which influence $\mathbf{y}$ but are not modeled by $\mathbf{x}$. Empirically, these factors seem to dominate $\mathbf{n}$, particularly for travel times. These factors would have some unknown correlation with respect to the various ray paths, but more seriously, they would be highly correlated over the time intervals between beacon transmissions as are the 
system parameters. This means that the estimate covariance would not converge to its theoretical "steady-state" value, rather the estimate would have a bias due to unmodeled features of the environment.

The data displayed is the estimate covariance after a single iteration of the Kalman filter rather than a steady state value. The least squares parameter estimate is [Gelb, 1989],

$$
\Delta \mathbf{x}=\mathrm{PC}^{\mathrm{T}}\left(\mathrm{CPC}^{\mathrm{T}}+\mathrm{R}\right)^{-1} \Delta \mathbf{y}
$$

and this parameter estimate has a covariance of

$$
P-P^{T}\left(C P C^{T}+R\right)^{-1} C P
$$

Written in this form, the covariance expression can be interpreted as the old parameter estimate covariance, $\mathrm{P}$, minus the reduction in covariance due to the measurements.

The covariance matrices for the updated parameter estimates were calculated on a $1 \mathrm{~km}$ grid over a $20 \mathrm{~km}$ square region for a three beacon network. Based on these covariances, the standard deviation of position is plotted in Section 4.2, for time synchronization in Section 4.3, and for the weights of the three EOFs in Section 4.4. Note from equation 4.1.3 that these covariances are deterministic functions of $\mathrm{P}, \mathrm{R}$, and $\mathrm{C}$ only. They are independent of the actual measurements. Their variability with position comes from the matrix $\mathrm{C}$, which depends on the eigenrays present and therefore changes with position. The matrices $\mathrm{P}$ and $\mathrm{R}$ are diagonal with diagonal elements given below:

- Initial Parameter Covariance -- $P$

$\mathrm{X}$ - and Y-Position: $(10 \mathrm{~m})^{2}$

Clock Error: $(1 \mathrm{sec})^{2}$

EOF Weights: $(10 \text { units })^{2}$
- Measurement Error Covariance -- $R$

Travel Times: $(1 \mathrm{~ms})^{2}$

Arrival Angles: $(1 \text { degree })^{2}$ 


\subsection{POSITION ESTIMATION ERROR}

The standard deviation of the position estimate (combined $\mathrm{x}$ - and y-errors) is shown below. It closely resembles in shape the Cramér - Rao bounds for position error calculated in Chapter 2. The vehicle and beacons are at 30m depth. The beacon positions are indicated by small circles.

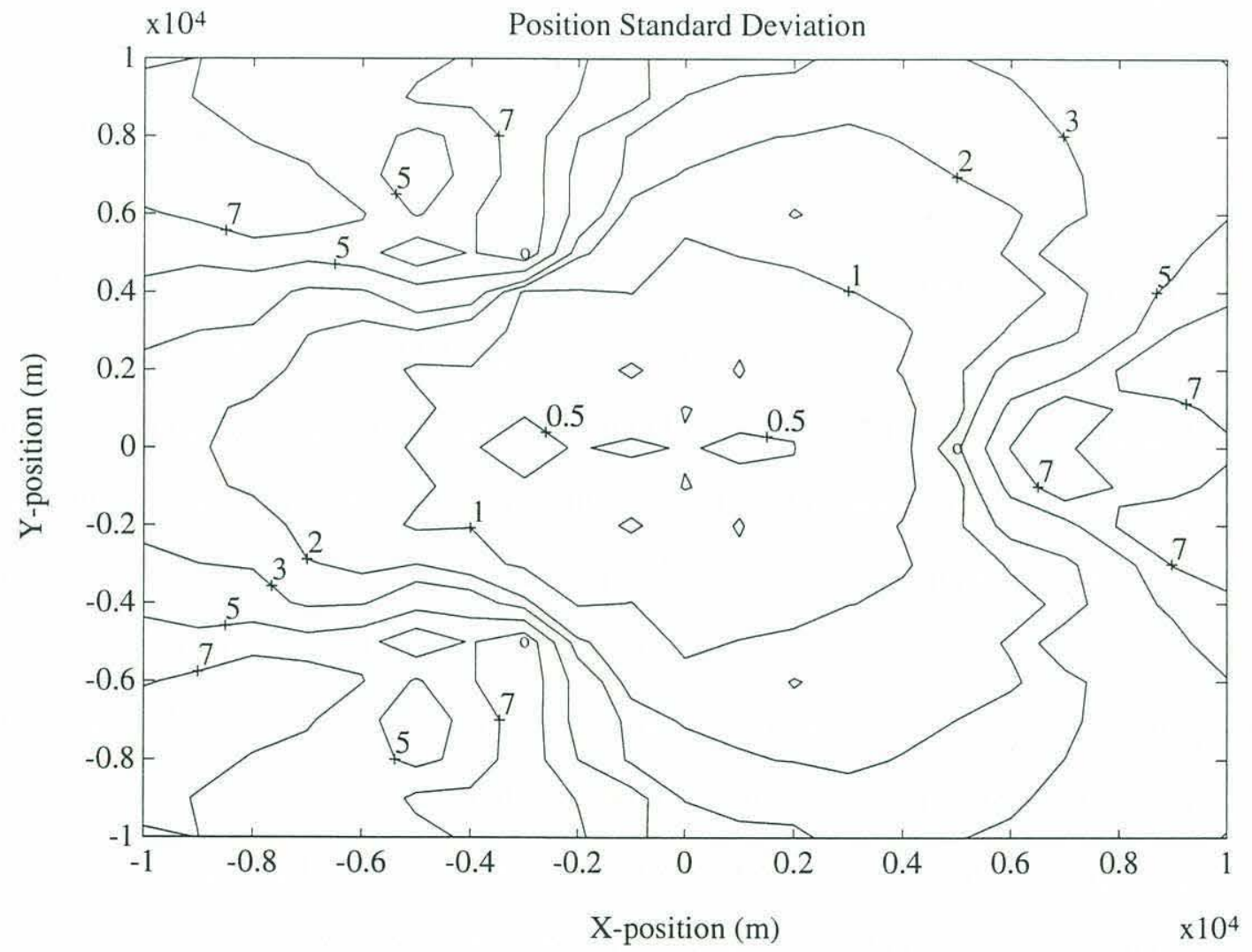

Figure 4.1: Standard deviation of position estimate. 


\subsection{TIME SYNCHRONIZATION ESTIMATION ERROR}

In discussing the Cramér-Rao bounds on position where the transmit time has varying levels of uncertainty, it was noted that the regions of greatest position sensitivity to time error were along the baseline extensions behind the beacons. These are the locations where large transmit time errors still produce consistent range measurements from the beacons, so the transmit time is less tightly constrained. The figure below demonstrates the large time errors along the baseline extensions. The vehicle and beacons are at $30 \mathrm{~m}$ depth. The beacon positions are indicated by small circles.

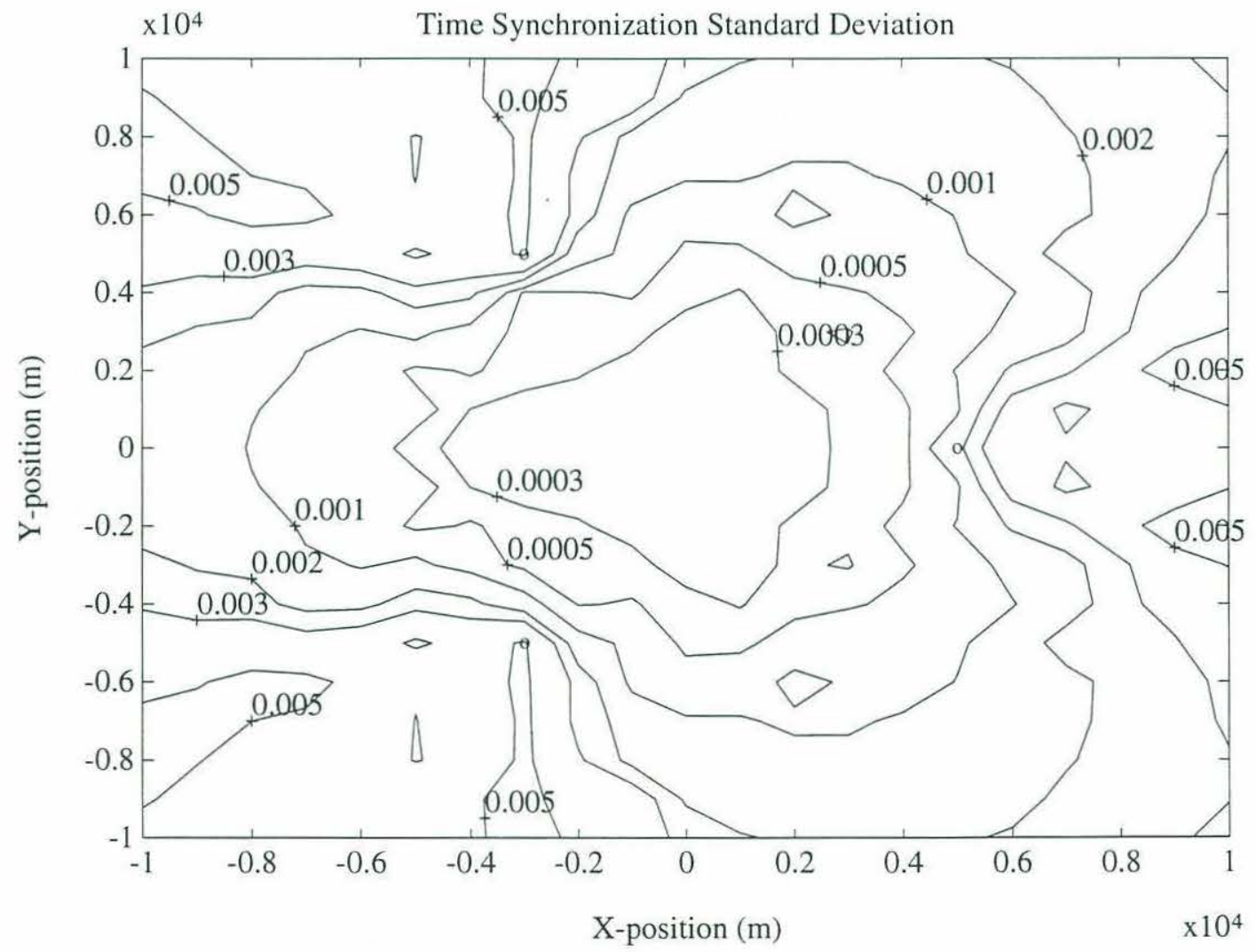

Figure 4.2: Standard deviation of time synchronization error. 


\subsection{ESTIMATION OF THE EOF WEIGHTS}

The standard deviation of the EOF weights is shown in Figures 4.3-4.5 for each of the three EOFs of Figure 2.11. These diagrams have a similar geometry to the Cramér-Rao bounds for profile parameter estimation. One of the interesting features of these errors is that the largest error results in the center of the beacon array, and the error decreases outside the beacon array. The great diversity of ray paths at longer ranges provides a more diverse sampling of the sound speed profile and therefore enables it to be sorted out into its component pieces with greater accuracy. At the very center, identical arrival structures are seen from each beacon, so the diversity of sampling of the sound speed profile is at its minimum. Additionally, at long ranges the travel time change for a given size velocity change is greater, making sound speed profile variations more noticeable over the measurement noise.

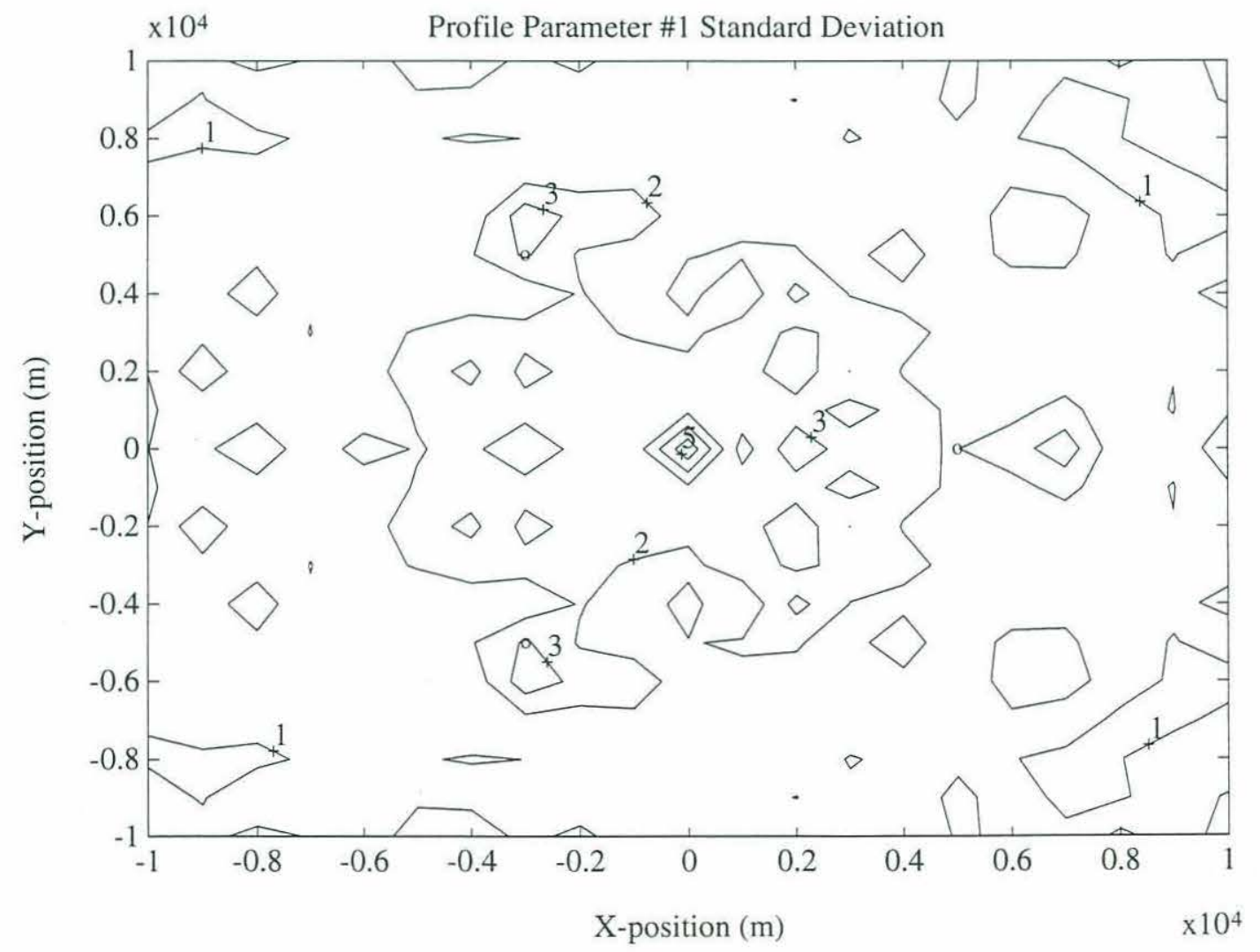

Figure 4.3: Standard deviation weight estimate for the first EOF of Figure 2.11. 


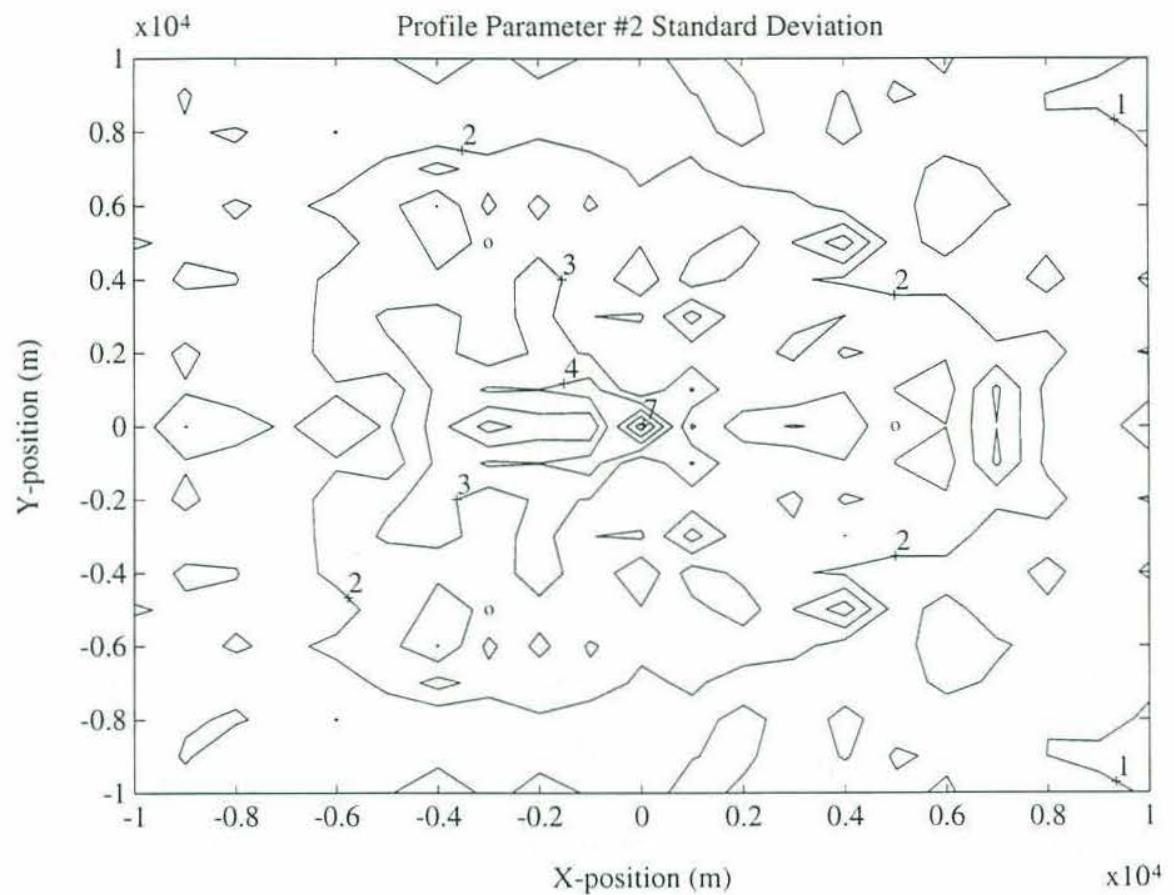

Figure 4.4: Standard deviation weight estimate for the second EOF of Figure 2.11 .

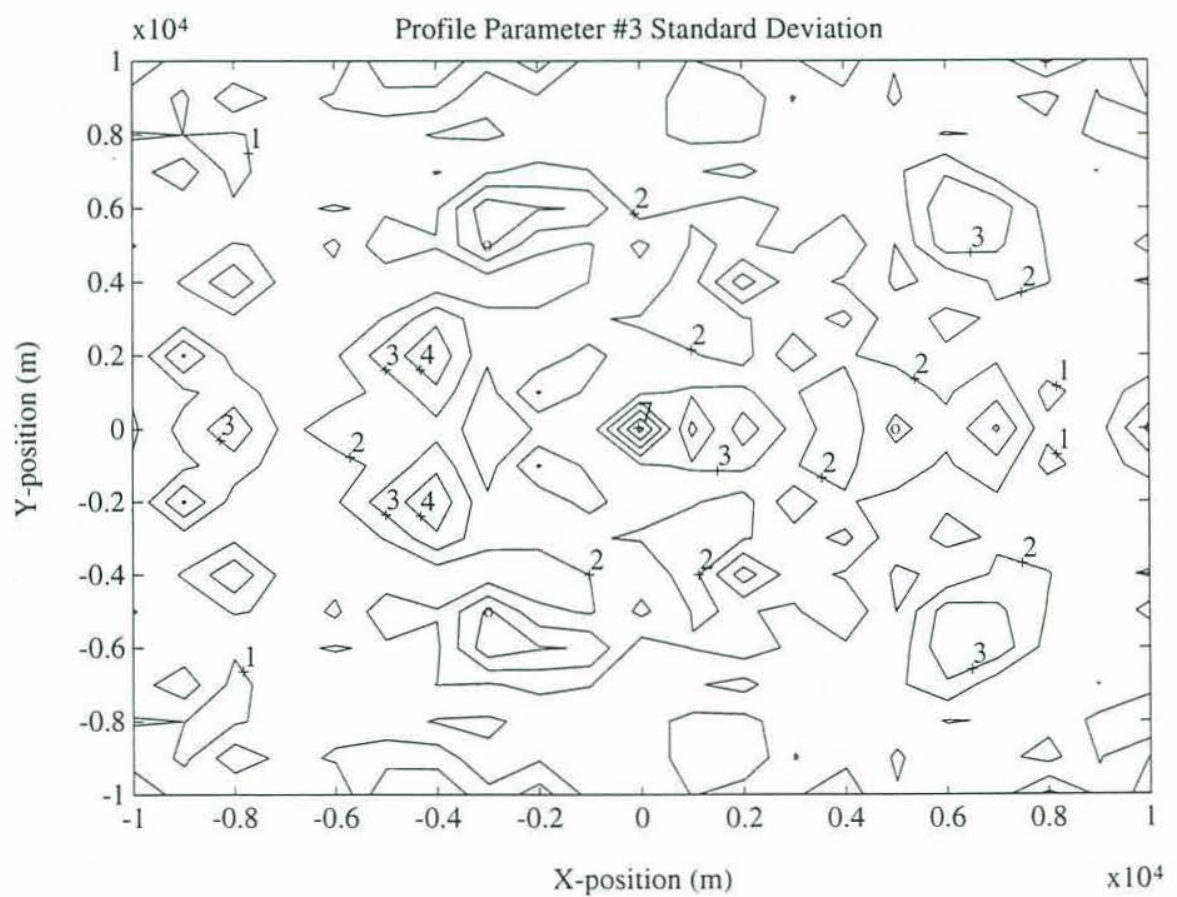

Figure 4.5: Standard deviation weight estimate for the third EOF of Figure 2.11 . 


\subsection{SUMMARY}

The observability of the various parameters depends on the rows of the matrix C. If the rows are close to orthogonal, then the parameters will be resolved well. It makes physical sense that this would be the case. First it is noted that the dominant source of information for the parameter calculation is travel time. The variations of angle with changes in parameter are too small to be of much help in the inversion, as discussed in Section 2.6. This relative immunity to parameter variations is, of course, the very property that makes angles so useful in path identification. Errors in the various sorts of parameters manifest themselves as errors of different geometries in the measurable quantities. A position change causes arrivals from some beacons to come in sooner and arrivals from other beacons to come in later independent of the beacon range. A change in time synchronization causes all the arrivals to come uniformly sooner or later. Finally, a sound speed change can cause a shift in acoustic travel times that is more or less proportional to range. Thus the geometries of travel time changes corresponding to the various parameters, or, in other words, the rows of $\mathrm{C}$, will tend to be orthogonal. It bodes well for the success of parameter estimation when the parameters are viewed through a matrix $\mathrm{C}$ which tends to have orthogonal columns.

The significance of the error results in Figures 4.1-4.5 should be kept in perspective. The calculated covariances are based on the ability to correctly match all the predicted arrivals. The larger the errors in the parameter set and the larger the measurement noise, the less likely a complete and correct matching becomes. Chapter 5 will examine the operation of the system in a realistic scenario, where ray paths must be identified before they can be used for position. 


\section{Chapter 5 Mission Example}

\subsection{INTRODUCTION}

This chapter will explore navigation performance under a realistic mission scenario. A comparison will be made between hyperbolic navigation with the assumption of a constant sound speed, a navigation system which uses a ray tracing model of the environment but only utilizes the first acoustic arrival for positioning, and the full multipath matching system. The simulations presented raise interesting issues about parameter resolvability, particularly with regards to the weights for the EOFs. 


\subsection{SAMPLE MISSION}

In the simulations that follow, the vehicle will be deployed at the center of the beacon network, at location $(0,0)$. The beacon network is shown in Figure 5.1. It will set a course at 30 degrees, and head in this direction at a rate of $2 \mathrm{~m} / \mathrm{s}$. The beacons will ping every 10 seconds, and the parameter errors will be plotted after receiving each of these pings. The error sources and error sizes which will be introduced are described below.

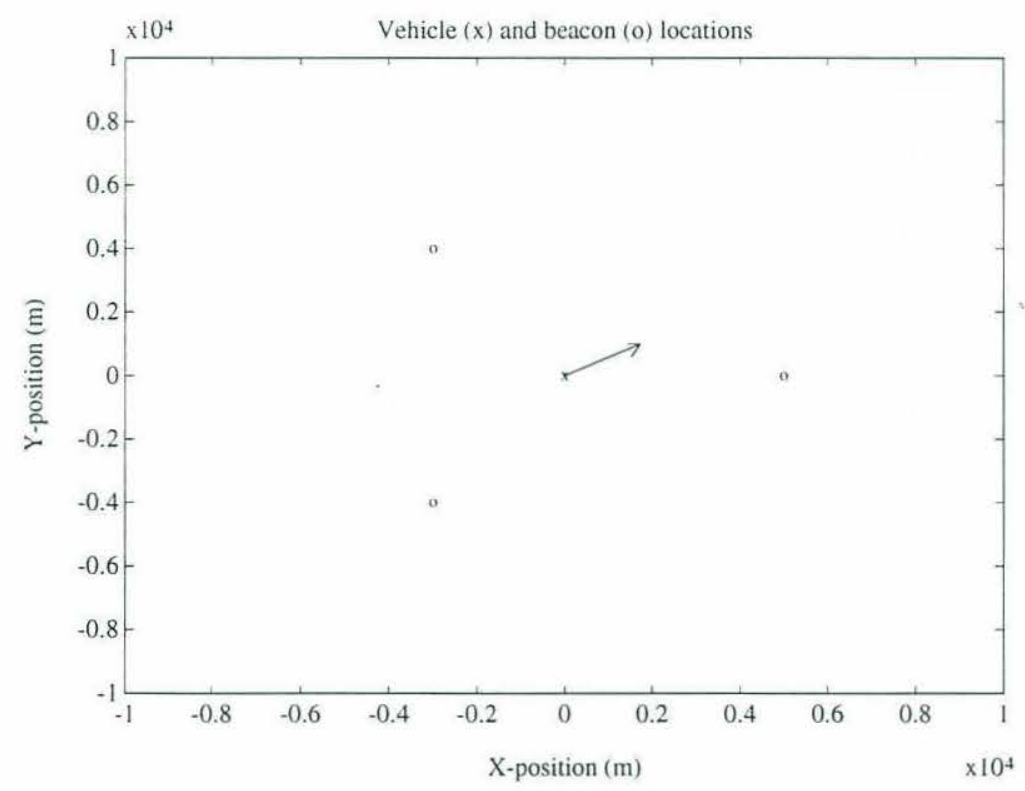

Figure 5.1: System test setup. Beacon locations are marked by 'o'. Vehicle location is marked by ' $x$ '. The arrow shows the direction of vehicle travel.

\section{- Position Error}

For the initial location of the vehicle, the position error will be $-20 \mathrm{~m}$ in the $\mathrm{x}$ direction and $10 \mathrm{~m}$ in the $y$-direction corresponding to typical GPS accuracies. In motion, the dead reckoning error from one time step to the next will have a standard deviation of $10 \%$ of the distance travelled between acoustic position fixes. Errors of this magnitude are reasonable for simple accelerometer-based dead reckoning. 


\section{- Timing Errors}

The initial timing error between the vehicle and beacon time will be assumed to be 1 second. This reflects a scenario where little prior effort has been made to synchronize the vehicle clock to beacon time. The vehicle time base error in measuring time intervals will have a standard deviation of 1 part in $10^{4}$, reflecting an uncompensated crystal oscillator [Horowitz and Hill, 1989].

\section{- Sound Speed Profile}

Recall that in Section 2.3, a series of 10 sound speed profiles collected over a five day period in the Greenland Sea was used to generate the EOFs. In this simulation, the true sound speed profile will be the 11 th profile in the series, that is, the first profile after the set used to generate the EOFs. This profile is shown in Figure 5.2. In these simulations, this profile is unknown to the vehicle and is constant over the duration of the mission.

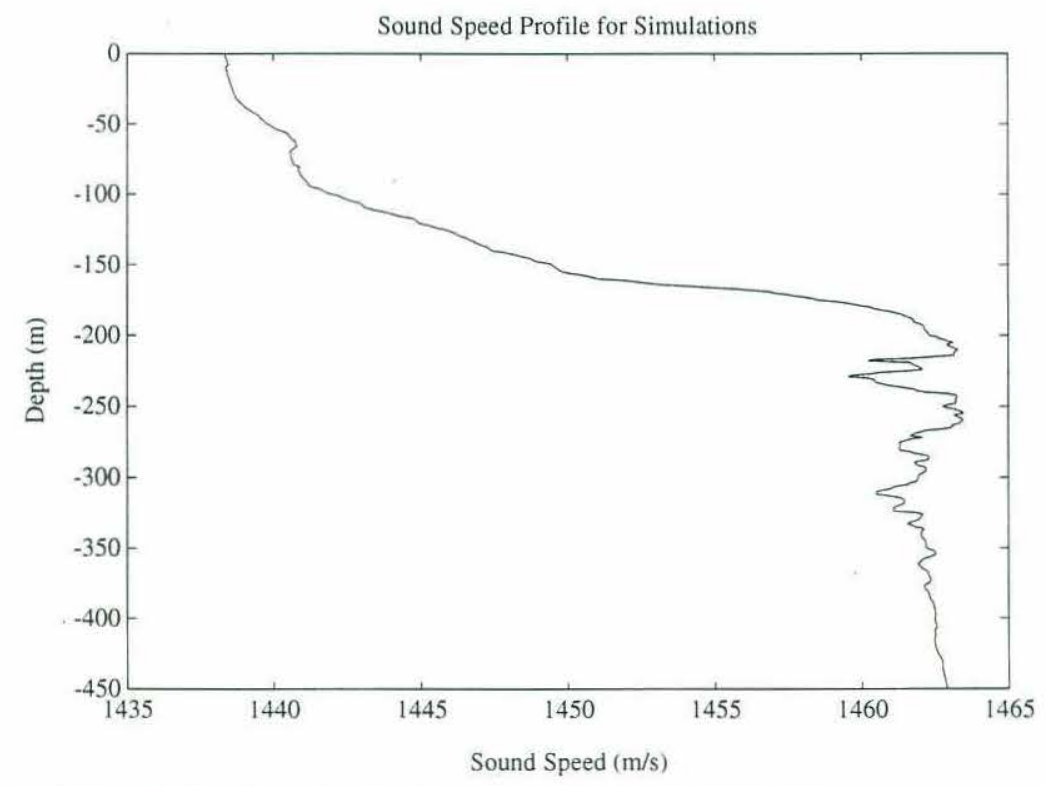

Figure 5.2: $\quad$ Sound speed profile used for system tests 
The measurement noise in travel time will be $1 \mathrm{~ms}$ and in arrival angle will be 1 degree. At the high frequencies used for navigation, these accuracies seem reasonable for the sort of equipment that might be caried by a small AUV. 


\subsection{POSITION ERRORS}

The position estimation errors in both $\mathrm{x}$ - and $\mathrm{y}$ - coordinates are given in Figures $5.3,5.4$, and 5.5 for the hyperbolic, first arrivals, and multipath matching systems only.

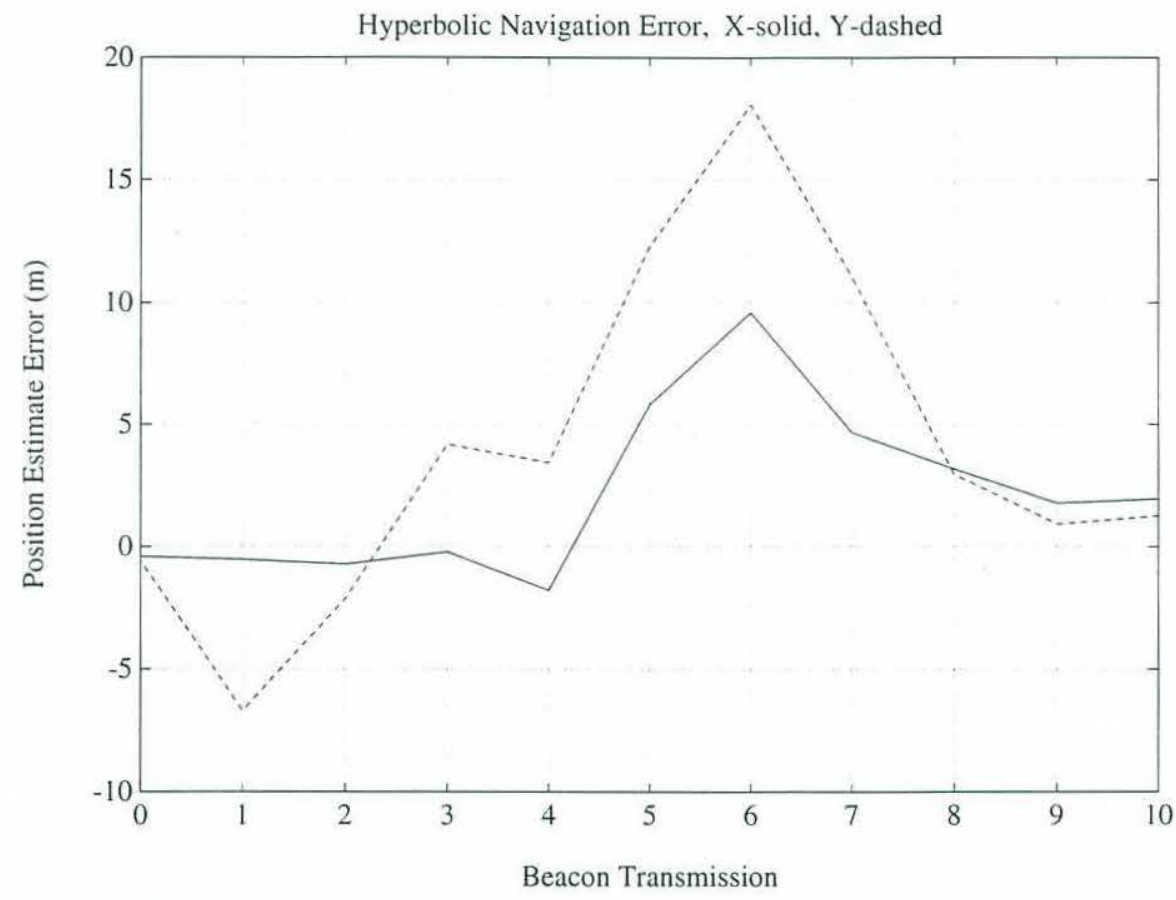

Figure 5.3: Position Estimation Error: Hyperbolic Navigation

The hyperbolic navigation system produced the largest errors, as might be expected, even though it was operating at the point in the beacon network where its poor modelling of sound propagation would have the smallest negative effect. The errors in travel times due to the assumption of straight ray paths would be equal from each beacon, and would therefore be eliminated by taking time differences since the vehicle is at the center of the network. 


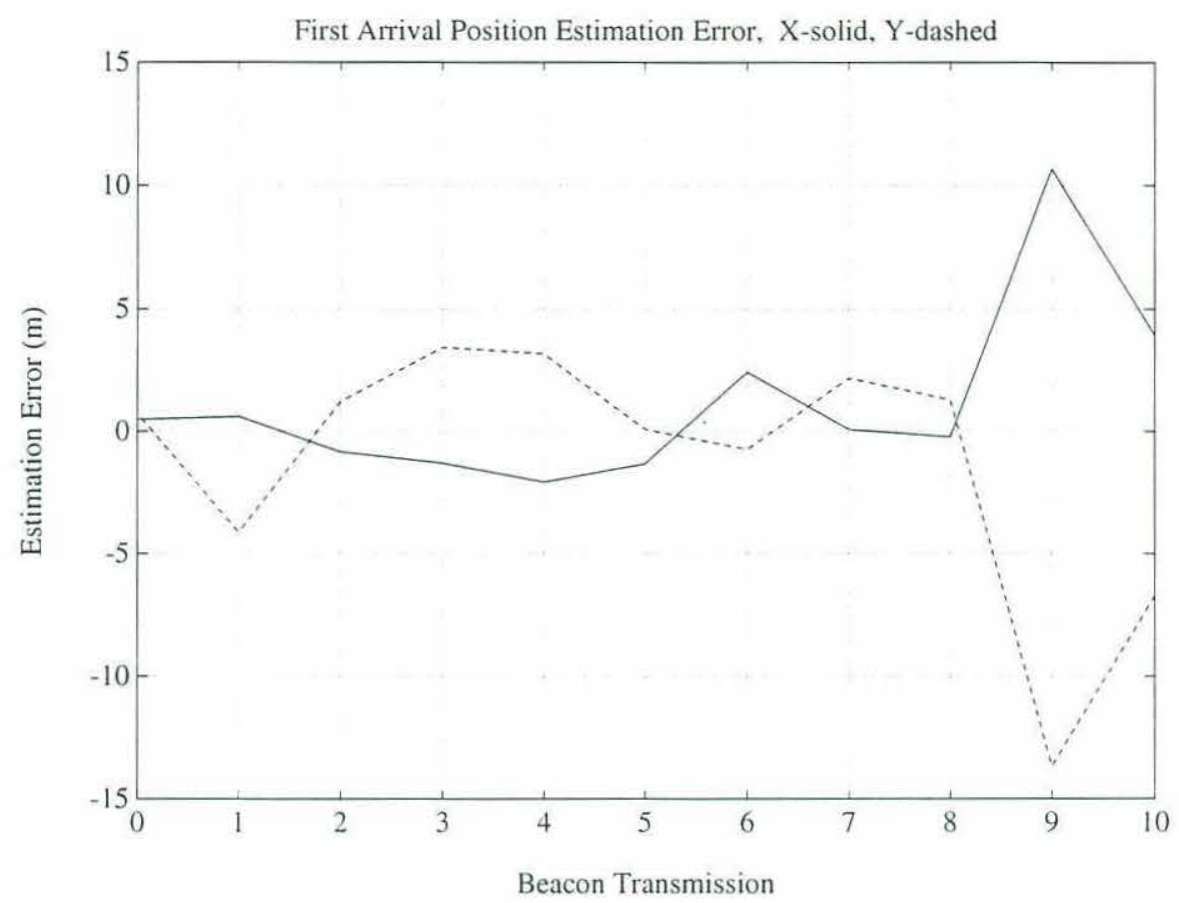

Figure 5.4: Position Estimation Error: First Arrival Matching

The first arrival matcher offers improved position accuracy because it estimates the time synchronization between the vehicle and the beacons as a random variable rather than simply removing it from the equations as does the hyperbolic system. The first arrival matcher also uses a ray tracing model of sound propagation for improved travel time prediction accuracy. Its weakness is that fading of the first arrival results in sudden position errors, as happened at beacon transmission number 9 in Figure 5.4. 


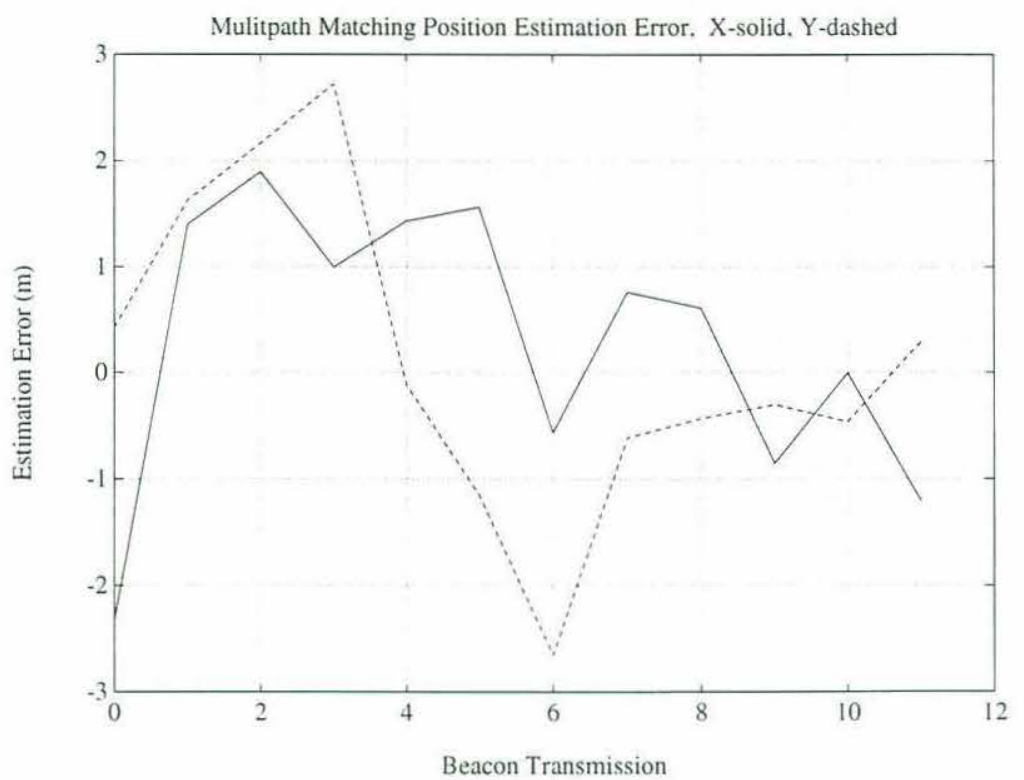

Figure 5.5: Position Estimation Error: Multipath Matching

The multipath matching system offers significant improvement in position accuracy over the hyperbolic system and the first arrival system. In particular, its use of many arrivals makes it robust to fading of some arrivals. 


\subsection{TIME SYNCHRONIZATION ERRORS}

The time synchronization between the beacons and the vehicle clock was estimated as a separate parameter by the first arrival system and the multipath matching system. The time synchronization error produced by both of these systems is shown below.

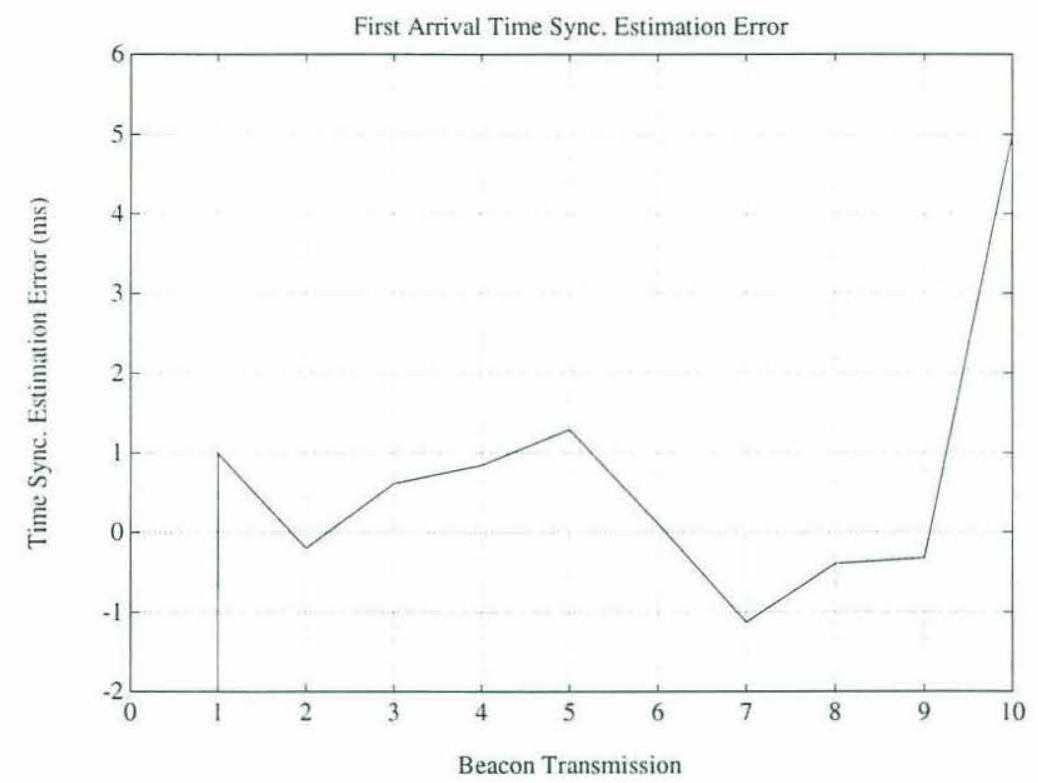

Figure 5.6: Time Synchronization Error: First Arrival Matching

The first arrival matching system showed spikes in synchronization error due to the problem of path fading. Otherwise it held synchronization to an accuracy of better than $1 \mathrm{~ms}$, as shown in Figure 5.6. 


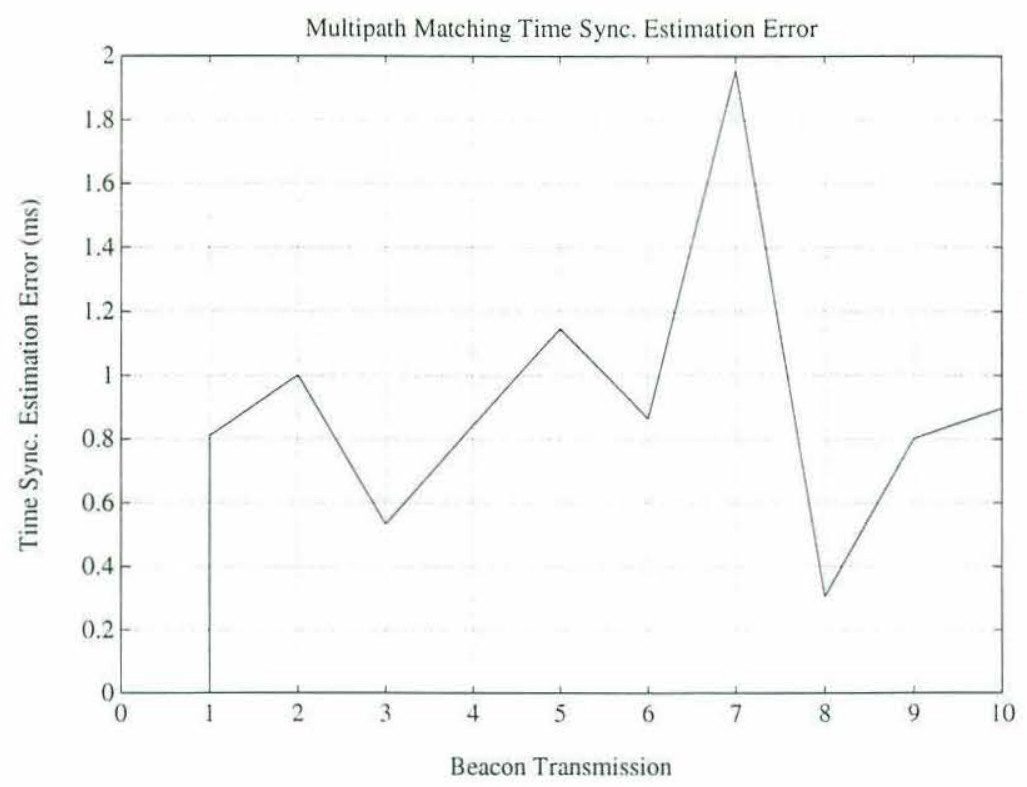

Figure 5.7: Time Synchronization Error: Multipath Matching

The multipath matching system also held accurate time synchronization. There seems to be a bias of about $1 \mathrm{~ms}$ in the time synchronization estimate. Such biases in the estimators are due to inaccuracies in the representation of the sound speed profile. It was noted in Section 4.5 that time synchronization errors could be separated from sound speed profile approximation errors because time synchronization errors are independent of beacon range, while profile approximation errors manifest themselves to first order as range dependent shifts in travel times. For the data in Figure 5.7, the vehicle was near the center of the beacon network, so range dependent shifts would be equal from all the beacons. This fact would lead to a coupling from sound speed profile errors to time synchronization errors at this vehicle position. 


\subsection{EOF WEIGHT ERRORS}

The multipath matching system estimated weights for the EOFs in an attempt to model the sound speed profile. As shown in Figure 5.8, these weights did not converge to their correct value.

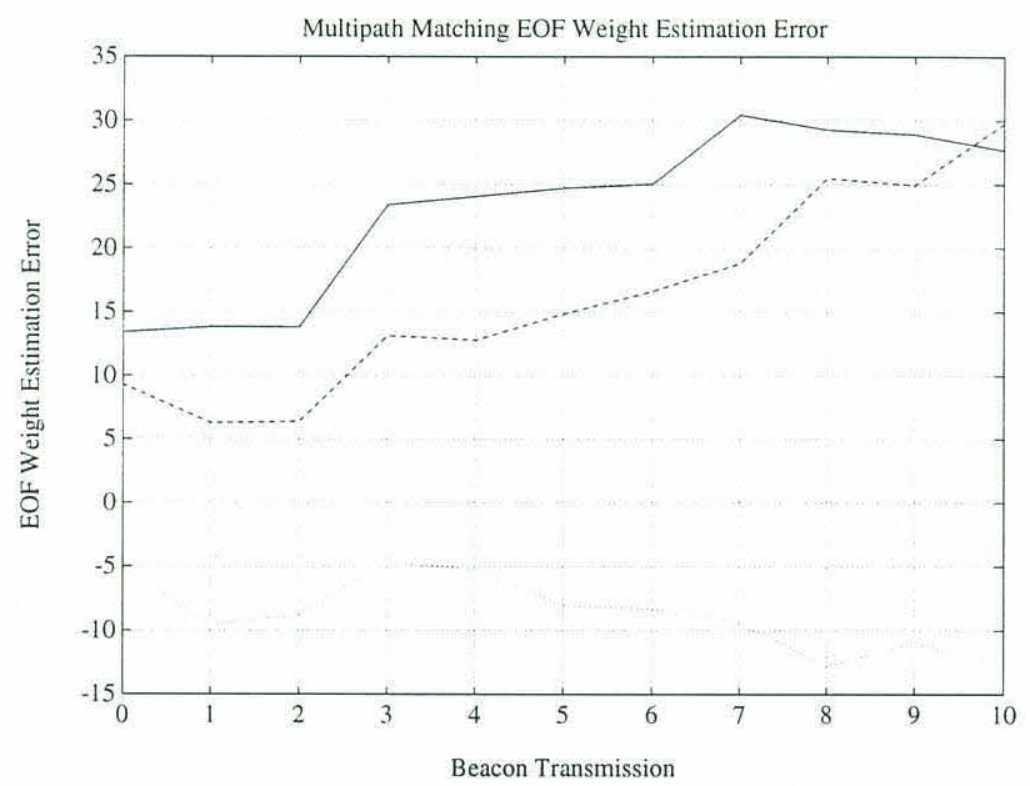

Figure 5.8: EOF Weight Estimation Error. The normal line is the error in the weight of the first EOF, dashed line of the second EOF, and dotted line of the third EOF.

There are several reasons that contribute to this failure to converge. First, placing the vehicle at the center of the navigation network meant that it was at the location where the EOF weights were least resolvable as shown in Figures 4.3-4.6. Second, although the EOFs are orthogonal vectors, their projections onto travel time perturbations need not be orthogonal. The ray paths will sample the EOFs most sensitively at just a few specific depths.

The estimate covariance calculated for the EOFs at the last beacon transmission indicated an expected standard deviation of 13 units for the first EOF, 16 units for the 
second EOF, and 18 units for the third EOF. The fact that the estimate covariance was so

large indicates that the weights are "wandering" because of an inability to resolve them, as opposed to converging to some incorrect value. Further evidence of this fact is found in the position and time synchronization estimate errors of Figures 5.5 and 5.7. If the measurable quantities depended in any strong way on the choice of EOF weights, then the position and time synchronization estimates would also be expected to diverge. This phenomenon of finding the wrong sound speed profile while still converging on an accurate position has been identified in the literature [Collins and Kuperman, 1991]. 



\section{Chapter 6 Conclusion}

The problem of acoustic navigation in a refractive fading multipath environment has been addressed. Substantial improvement in position accuracy was demonstrated relative to systems which use only the first arrival when the first arrival is subject to fading. Employing the physics of how individual rays behave with changes in parameter values offers a significant improvement in computational speed for finding the parameter set that best predicts the observed signal. This makes possible the implementation of this matched field approach to positioning with the relatively modest computational resources that a small AUV could carry and power.

Work in the immediate future will focus on testing the assumptions made in Section 2.1. One of the central requirements for multipath navigation is the ability to accurately predict and measure the received acoustic signal. This spring, acoustic beacons will be deployed through the Arctic ice pack and their transmissions will be digitized at ranges up to $10 \mathrm{~km}$. This data will aid in determining what set of parameters are most important in describing the environment.

The parameter inversion accuracy results of Chapter 4 indicated great promise for the observability of the parameter values. Chapter 5 demonstrated that these theoretical accuracies can only be realized when sufficiently large numbers of rays can be properly identified. As the uncertainties in arrival position increase, more measured arrivals lie in the ambiguity region of each predicted arrival, and the probability of correct matching decreases. Future theoretical work on this topic will include exploration of the limitations on parameter estimate accuracy in light of the difficulty in arrival matching. 

slowly in time, like the sound speed profile, an old estimate of their values at a location where they are very observable may be more accurate than a current estimate if the current location is one for which the parameter is not very observable. An interesting extension of this work would be to consider the question of which path a vehicle should follow between two points so that the information it accumulates along the way gives it the most accurate possible estimate of its position when it reaches its destination. 


\section{Appendix A:}

\section{Fast Ray Tracing}

The ray tracing problem can be divided into two parts. The first, and most time consuming, is finding eigenrays, that is, the ray paths which connect the transmitter and the receiver. The second ray tracing problem is to calculate the travel times along the eigenrays as well as calculating the various derivatives necessary for the inversion for parameter values. The derivative calculations are covered in Appendix B. The finding of eigenrays is covered here.

The search for eigenrays is a matter of determining the initial ray angles which solve the ray depth equation at the transmitter range:

$$
\begin{aligned}
& Z_{r}-Z_{t}=\int_{X t}^{X r} \tan a(x) d x \\
& \frac{d \tan a(x)}{d x}=\left(\frac{d}{d c} \frac{ \pm \sqrt{c_{t}^{2}-c^{2}}}{c}\right) \frac{d c}{d z} \frac{d z}{d x} \\
&=\frac{-c_{t}^{2}}{c^{3}} \frac{d c}{d z}
\end{aligned}
$$




$$
\begin{aligned}
& \text { Where } \quad \begin{array}{l}
Z_{r} \text { is the receiver depth } \\
Z_{t} \text { is transmitter depth } \\
X_{r} \text { is receiver horizontal range } \\
X_{t} \text { is transmitter horizontal range } \\
a \text { is the ray angle below the horizontal }
\end{array}
\end{aligned}
$$

Turning these ray equations into difference equations yields

$$
\begin{gathered}
Z_{n+1}=Z_{n}+\tan a_{n} D x \\
\tan a_{n+1}=\tan a_{n}+\frac{-c_{t}^{2}}{c\left(Z_{n}\right)^{3}} \frac{d c\left(Z_{n}\right)}{d z}
\end{gathered}
$$

The change in the tangent of $a$ depends only on depth, so it can be evaluated ahead of time. In this way, finding the depth at receiver range of a ray requires two sums and a table lookup per horizontal distance step. 


\section{Appendix B Derivatives}

The relationship between changes in parameters and changes in measurable quantities is linearized around the current estimate of the parameter values. Let the following definitions be made,

$$
\begin{array}{ll}
\mathbf{x}_{\mathbf{0}} & \text { Estimated model parameter vector. } \\
\mathbf{y}_{\mathbf{0}} & \text { Estimated measurables vector, corresponding to } \mathbf{x}_{\mathbf{0}} . \\
\mathbf{x}_{\mathbf{0}}+\Delta \mathbf{x} & \text { True model parameter vector, where } \Delta \mathbf{x} \text { is the estimation error. } \\
\mathbf{y}_{\mathbf{0}}+\Delta \mathbf{y} & \text { True measurables vector, where } \Delta \mathbf{y} \text { is the measurement } \\
& \text { prediction error produced by } \Delta \mathbf{x} .
\end{array}
$$

The relationship $\mathbf{y}=f(\mathbf{x})$ is non-linear, however once a point $\mathbf{y}_{\mathbf{0}}=f\left(\mathbf{x}_{\mathbf{0}}\right)$ on this curve is established, the local linearization can be made:

$$
\Delta \mathbf{y}=\mathrm{C} \Delta \mathbf{x}
$$

The matrix $\mathrm{C}$ is then the matrix of partial derivatives of the elements of $\mathbf{y}$ with respect to the elements of $\mathbf{x}$ evaluated at $\mathbf{x}_{\mathbf{0}}$,

$$
\mathrm{C}_{i j}=\frac{\partial y_{i}}{\partial x_{j}}
$$


The elements of this matrix of partial derivatives are calculated below, but first, some variables are be defined.

\section{Transmitter}

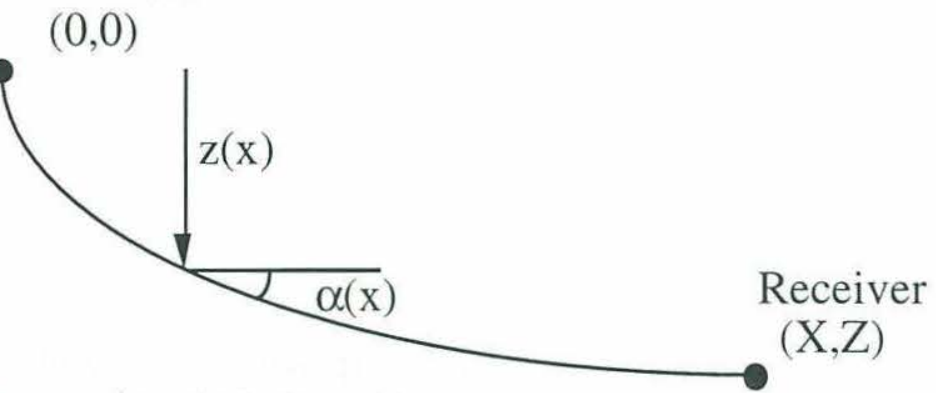

Figure B.1: Definition of terms

Z Vertical distance from transmitter to receiver

X Horizontal distance from transmitter to receiver

$z(x) \quad$ Depth of the ray at a horizontal range $\mathrm{x}$ from the transmitter

$\alpha(x) \quad$ Ray angle at horizontal range $\mathrm{x}$ from the transmitter

$\zeta(x) \quad$ Rate of change of depth at horizontal range $\mathrm{x}$

$\xi(z) \quad$ Rate of change of range at a particular depth point along the eigenray

$\tau(x) \quad$ Rate of change of total travel time $\mathrm{t}$ with respect to horizontal range at $\mathrm{x}$

$t \quad$ Travel time from transmitter to receiver

$T \quad$ Clock error in the vehicle clock

The equations below follow from the above definitions,

$$
\begin{gathered}
t=\int_{0}^{X} \tau(x) d x \\
Z=\int_{0}^{X} \zeta(x) d x
\end{gathered}
$$




$$
X=\int_{0}^{Z} \xi(z) d z
$$

The estimated sound speed profile is,

$$
c(z)=c_{0}(z)+p \phi(z)
$$

where $\phi(z)$ is one of the EOFs generated in Section 2.3 and $p$ is the weight of the EOF.

The linearized variations for travel time and arrival angle can be written, for clock synchronization error $T$.

$$
\begin{gathered}
d t=\left(\frac{\partial t}{\partial X}\right)_{p} d X+\left(\frac{\partial t}{\partial p}\right)_{X} d p+T \\
d \alpha=\left(\frac{\partial \alpha}{\partial X}\right)_{p} d X+\left(\frac{\partial \alpha}{\partial p}\right)_{X} d p
\end{gathered}
$$




\section{DERIVATIVES WITH RESPECT TO $\mathrm{X}$}

- CAlCUlation OF $\left(\frac{\partial \mathrm{t}}{\partial \mathrm{X}}\right)_{p}$

The derivative of travel time with respect to horizontal range $X$ is [Hildebrandt, 1976],

$$
\begin{gathered}
\left(\frac{\partial t}{\partial X}\right)_{p}=\frac{\partial}{\partial X} \int_{0}^{X} \tau(x) d x \\
=\tau(\mathrm{X})+\int_{0}^{X}\left(\left(\frac{\partial \tau}{\partial c_{t}} \frac{\mathrm{dc} t}{\mathrm{dX}}\right)+\left(\frac{\partial \tau}{\partial c} \frac{\mathrm{dc}}{\mathrm{dX}}\right)\right) d x \\
=\tau(\mathrm{X})+\int_{0}\left(\left(\frac{\partial \tau}{\partial c_{t}} \frac{\mathrm{dc} t}{\mathrm{dX}}\right)+\left(\frac{\partial \tau}{\partial c} \frac{\partial \mathrm{c}}{\partial \mathrm{z}} \frac{\mathrm{dz}}{\mathrm{dX}}\right)\right) d x
\end{gathered}
$$

Evaluating the first regular derivative

$$
\begin{gathered}
\frac{d c_{t}}{d X}=\left(\frac{d X}{d c_{t}}\right)^{-1} \\
X=\int_{0}^{Z} \xi\left(z^{\prime}\right) d z^{\prime} \\
\frac{d X}{d c_{t}}=\int_{0}^{Z}\left(\frac{\partial \xi(z)}{\partial c_{t}}+\frac{\partial \xi(z)}{\partial c} \frac{d c(z)}{d c_{t}}\right) d z
\end{gathered}
$$


Changing the variable of integration to $x$,

$$
\begin{gathered}
\frac{d X}{d c_{t}}=\int_{0}^{X}\left(\frac{\partial \xi(z)}{\partial c t}+\frac{\partial \xi(z)}{\partial c} \frac{d c}{d c_{t}}\right) \tan \theta(x) d x \\
=\int_{0}^{X}\left(\frac{\partial \xi(z)}{\partial c t}+\frac{\partial \xi(z)}{\partial c} \frac{\partial c(z)}{\partial z} \frac{d z}{d c_{t}}\right) \tan \theta(x) d x \\
\frac{d z(x)}{d c_{t}}=\int_{0}^{x} \frac{\partial \zeta\left(x^{\prime}\right)}{\partial c_{t}}+\frac{\partial \zeta\left(x^{\prime}\right)}{\partial c} \frac{\partial c\left(z^{\prime}\right)}{\partial z^{\prime}} \frac{d z^{\prime}}{d c_{t}} d x^{\prime}
\end{gathered}
$$

Evaluating the second regular derivative

$$
\begin{gathered}
\frac{d z}{d X}= \\
z=\int_{0}^{x} \zeta\left(x^{\prime}\right) d x^{\prime} \\
\frac{d z}{d X}=\int_{0}^{x} \frac{\partial \zeta(x)}{\partial c_{t}} \frac{d c_{t}}{d X}+\frac{\partial \zeta(x)}{\partial c} \frac{\partial c\left(x^{\prime}\right)}{\partial z} \frac{d z^{\prime}\left(x^{\prime}\right)}{d X} d x
\end{gathered}
$$

\section{- CAlCUlation OF $\left(\frac{\partial \alpha}{\partial X}\right)_{p}$}

The derivative of arrival angle with respect to vehicle position is changed to a derivative of turning velocity. 


$$
\left(\frac{\partial \alpha}{\partial X}\right)_{p}=\left(\begin{array}{ll}
\frac{\partial \alpha}{\partial c_{t}} & \frac{d c_{t}}{d X}
\end{array}\right)_{p}
$$

$$
c_{t}=\frac{c(x)}{\cos \alpha(x)}
$$

$$
\frac{\partial c_{t}}{\partial \alpha}=\frac{c(x) \sin \alpha(x)}{\cos ^{2} \alpha(x)}
$$

The $\frac{d c_{t}}{d X}$ term was found above already. 


\section{DERIVATIVES WITH RESPECT TO $p$}

\section{- CAlCulation OF $\left(\frac{\partial t}{\partial p}\right)_{X}$}

The derivative of time with respect to sound speed profile parameter is,

$$
\begin{aligned}
& \left(\frac{\partial t}{\partial p}\right)_{S_{R}}=\frac{\partial}{\partial p} \int_{0}^{S_{R}} \frac{1}{c(s)} d s \\
& =\int_{0}^{S_{R}} \frac{\partial \sigma}{\partial p}+\frac{\partial \sigma}{\partial s} \frac{d s}{d p} d s
\end{aligned}
$$

The slowness depends only on depth in this horizontally stratified medium,

$$
=\int_{0}^{S_{R}} \frac{\partial \sigma}{\partial p}+\frac{\partial \sigma}{\partial z} \frac{d z}{d p} d s
$$

To evaluate the path integral, it is changed into an integral along horizontal range $\mathrm{x}$,

$$
=\int_{0}^{X}\left(\frac{\partial \sigma}{\partial p}+\frac{\partial \sigma}{\partial z} \frac{d z}{d p}\right) \frac{1}{\cos \theta(\mathrm{x})} d x
$$

The first term in the brackets corresponds to the change in travel time along the old ray path from a change in sound speed profile. The second term corresponds to the effect of change in path due to a change in sound speed profile. Fermat's principle states that the ray path is an extremum of time with respect to all possible paths from source to receiver. The 
implication of this for variation of time with respect to sound speed perturbations is that the integral of time along the old ray path through the new medium is equal to the integral of time along the new ray path through the new medium. The terms due to a differential change in path will integrate to zero, leaving,

$$
\begin{gathered}
=\int_{0}^{X} \frac{\partial \sigma}{\partial p} \frac{1}{\cos \theta(\mathrm{x})} d x \\
\frac{\partial \sigma}{\partial z}=\frac{-1}{c^{2}} \frac{\partial c}{\partial z}
\end{gathered}
$$

- Calculation of $\left(\frac{\partial \alpha}{\partial p}\right)_{X}$

As with the derivative of time with respect to sound speed profile parameter, a conversion is made from $\alpha$ to $c_{t}$, since the relevant equations are in terms of the latter.

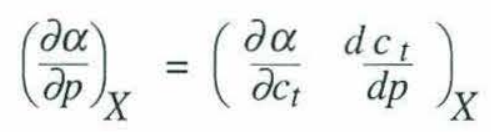

To calculate the regular derivative with respect to $\mathrm{p}$, the $\mathrm{X}$ and $\mathrm{Z}$ positions of transmitter and receiver are kept fixed. The sound speed profile parameter $\mathrm{p}$ and the ray turning velocity ct will vary together in such a way as to keep the ultimate destination of the ray the same. This gives the relationship, 


$$
\frac{d Z}{d p}=0, \quad \text { so } \quad \frac{\partial Z}{\partial p}+\frac{\partial Z}{\partial c_{t}} \frac{d c_{t}}{d p}=0
$$

$$
\frac{d c_{t}}{d p}=-\frac{\frac{\partial Z}{\partial p}}{\frac{\partial Z}{\partial c_{t}}}
$$

$$
\text { using, } \quad Z=\int_{0}^{X} \zeta(x) d x
$$

$$
=-\frac{\int_{0}^{X} \frac{\partial \zeta(x)}{\partial c} \frac{d c}{d p} d x}{\int_{0}^{X} \frac{\partial \zeta(x)}{\partial c_{t}} d x}
$$

$$
\frac{d c(x)}{d p}=\left(\frac{\partial c}{\partial p}+\frac{\partial c}{\partial z} \frac{d z}{d p}\right)
$$

$$
=-\frac{\int_{0}^{X} \frac{\partial \zeta(x)}{\partial c}\left(\frac{\partial c}{\partial p}+\frac{\partial c}{\partial z} \frac{d z}{d p}\right) d x}{\int_{0}^{X} \frac{\partial \zeta(x)}{\partial c_{t}} d x}
$$

$$
\begin{gathered}
\frac{d z(x)}{d p}=\int_{0}^{x} \frac{\partial \zeta\left(x^{\prime}\right)}{\partial c\left(x^{\prime}\right)} \frac{d c\left(x^{\prime}\right)}{d p}+\frac{\partial \zeta\left(x^{\prime}\right)}{\partial c_{t}} \frac{d c_{t}}{d p} d x^{\prime} \\
\frac{d z(x)}{d p}=\int_{0}^{x} \frac{\partial \zeta\left(x^{\prime}\right)}{\partial c\left(x^{\prime}\right)}\left(\frac{\partial c}{\partial p}+\frac{\partial c}{\partial z} \frac{d z\left(x^{\prime}\right)}{d p}\right)+\frac{\partial \zeta\left(x^{\prime}\right)}{\partial c_{t}} \frac{d c_{t}}{d p} d x^{\prime}
\end{gathered}
$$


This shows the unfortunate result that $d c t / d p$ is a function of $d z / d p$ at each $z(x)$ along the ray path, and $d z / d p$ is a function of dct/dp and of itself at each prior point along the ray path. Solving this system becomes a matter of plugging in guesses for $d c t / d p$ until the condition $d Z(X) / d p=0$ is met. For an initial guess, the empirical observation is made that

$$
\begin{gathered}
\frac{d c(x)}{d p}=\frac{\partial c}{\partial p}+\frac{\partial c}{\partial z} \frac{d z}{d p} \approx \frac{\partial c}{\partial p} \\
\frac{d c_{t}}{d p} \approx-\frac{\int_{0}^{X} \frac{\partial \zeta(x)}{\partial c} \frac{\partial c}{\partial p} d x}{\int_{0}^{X} \frac{\partial \zeta(x)}{\partial c_{t}} d x}
\end{gathered}
$$

This value is then used in the integral to check the validity of the guess.

$$
\begin{gathered}
\frac{d Z(x)}{d p}=0 \\
=\int_{0}^{X} \frac{\partial \zeta\left(x^{\prime}\right)}{\partial c\left(x^{\prime}\right)} \frac{\partial c}{\partial p}+\frac{\partial \zeta\left(x^{\prime}\right)}{\partial c\left(x^{\prime}\right)} \frac{\partial c}{\partial z} \frac{d z\left(x^{\prime}\right)}{d p}+\frac{\partial \zeta\left(x^{\prime}\right)}{\partial c_{t}} \frac{d c_{t}}{d p} d x^{\prime}
\end{gathered}
$$

A second slightly different guess is made, a slope between the two errors is calculated, and an improved estimate is made. One iteration of this procedure seems to provide a sufficiently accurate estimate of the derivative. 


\section{EVALUATION OF THE PARTIAL DERIVATIVES}

The partial derivatives are given in terms of $\tan \theta$ since that is the quantity calculated directly with each horizontal distance step in the ray tracing software.

$$
\begin{aligned}
& \tau(x)=\frac{1}{c \cos (z)}=\frac{c_{t}}{c^{2}(z)} \\
& \frac{\partial \tau(x)}{\partial c}=\frac{-2 c_{t}}{c^{3}(z)} \quad \frac{\partial \tau(x)}{\partial c_{t}}=\frac{1}{c^{2}(z)} \\
& \zeta(x)=\tan \theta(\mathrm{x})=\frac{ \pm \sqrt{c_{t}^{2}-c^{2}}}{c} \\
& \frac{\partial \zeta(x)}{\partial c}=-\frac{ \pm c_{t}{ }^{2}}{c^{2} \sqrt{c_{t}^{2}-c^{2}}}=\frac{-c_{t}{ }^{2}}{c^{3} \tan \theta} \\
& \frac{\partial \zeta(x)}{\partial c_{t}}=\frac{ \pm c_{t}}{c \sqrt{c_{t^{2}}-c^{2}}}=\frac{c_{t}}{c^{2} \tan \theta} \\
& \xi(z)=\frac{1}{\tan \theta(x)}=\frac{c}{ \pm \sqrt{c_{t}^{2}-c^{2}}} \\
& \frac{\partial \xi(z)}{\partial c}=\frac{c_{t}^{2}}{ \pm\left(c_{t}^{2}-c^{2}\right)^{3 / 2}}=\frac{c_{t}^{2}}{c^{3} \tan ^{3} \theta} \\
& \frac{\partial \xi(z)}{\partial c_{t}}=-\frac{c c_{t}}{ \pm\left(c_{t}^{2}-c^{2}\right)^{3 / 2}}=-\frac{c_{t}}{c^{2} \tan ^{3} \theta}
\end{aligned}
$$


116

Using these values for the partial derivatives, the ray integrals above can be evaluated numerically. 


\section{Bibliography}

Bellingham, James G., et al., "Arctic Under-Ice Survey Operations," Proc. 8th Intl. Conf. on Unmanned Untethered Submersible Technology, 50-59 (1993).

Bellingham, James G., et al., "Hyperbolic Acoustic Navigation for Underwater Vehicles: Implementation and Demonstration," Proc. IEEE AUV'92 (1992).

Bunday, Brian D., Basic Linear Programming (Edward Arnold, Baltimore, MD, 1984).

Burdic, William S., Underwater Acoustic System Analysis, 2nd ed. (Prentice Hall, Englewood Cliffs, NJ, 1991).

Catipovic, Josko A. and Arthur B. Baggeroer, "Analysis of high-frequency multitone transmissions propagated in the marginal ice zone," J. Acoust. Soc. Am. 88, 185189 (1990).

Cestone, J.A., R.J. Cyr, Gordon Roesler, and E. St.George, "Recent Developments in Acoustic Underwater Navigation," J. Navigation 30, 246-280 (1977).

Coates, Rodney and Liansheng Wang, "The 'WISP' Within-Structure Positioning System," Proc. 8th Intl. Conf. on Unmanned Untethered' Submersible Technology, 231-238 (1993).

Collins, Michael D. and W.A. Kuperman, "Focalization: Environmental focusing and source localization," J. Acoust. Soc. Am. 90, 1410-1422 (1991).

Cornuelle, B., W. Munk, and P. Worchester, "Ocean Acoustic Tomography from Ships," J. Geophys. Res. 94, 6232-6250 (1989).

Davis, Russ E., "Predictability of Sea Surface Temperature and Sea Level Pressure Anomalies over the North Pacific Ocean," J. Phys. Ocean. 6, 249-266 (1976).

Deffenbaugh, Max, et al., "Acoustic Navigation for Arctic Under-Ice AUV Missions," Proc. Oceans '93 (1993).

Duckworth, G. L., "A Robust Algorithm for Tracking of Drifting Acoustic Arrays in the Arctic," Conference Record: Twenty-first Asilomar Conference on Signals, Systems, \& Computers. 103-108 (1987).

Flatté, S. M., et al., Sound Transmission Through a Fluctuating Ocean (Cambridge University Press, New York, 1979). 
Frisk, George V., Ocean and Seabed Acoustics (Prentice-Hall, Englewood Cliffs, NJ, 1994).

Hassab, J. C., "Passive Tracking of a Moving Source by a Single Observer in Shallow Water," J. Sound and Vibration. 44, 127-145 (1976).

Hildebrandt, Francis B., Advanced Calculus for Applications (Prentice-Hall, Inc., Englewood Cliffs, NJ, 1976).

Horowitz, Paul and Winfield Hill, The Art of Electronics, 2nd ed. (Cambridge University Press, New York, 1989).

Howe, Bruce M., Peter F. Worchester, and Robert C. Spindel, "Ocean Acoustic Tomography: Mesoscale Velocity," J. Geophys. Res. 92 3785-3805 (1987).

Hurdle, B. G., The Nordic Seas, (Springer-Verlag, New York, 1986).

Ignizio, James, P., Linear Programming in Single- \& Multiple-Objective Systems (Prentice-Hall, Englewood Cliffs, NJ, 1982).

Mercer, J. A., and J. R. Booker, "Long-Range Propagation of Sound Through Oceanic Mesoscale Structures," J. Geophys. Res. 88 689-699 (1983)

Milne, P. H., Underwater Acoustic Positioning Systems (E. \& F. N. Spon, Ltd., New York, 1983).

Nahi, N. E., Estimation Theory and Applications (John Wiley and Sons, Inc., New York, 1969).

Pedersen, Melvin A. "Acoustic Intensity Anomalies Introduced by Constant Velocity Gradients." J. Acoust. Soc. Am. 33, 465-474 (1961).

Pedersen, Melvin A. and David F. Gordon. "Comparison of Curvilinear and Linear Profile Approximation in the Calculation of Underwater Sound Intensities by Ray Theory." J. Acoust. Soc. Am. 41, 419-438 (1967).

Scheer, Edward, XBT casts from CEAREX '89, personal communication.

Schmidt, Henrik, personal communication.

Sonnenberg, G. J., Radar and Electronic Navigation, 6th ed. (Butterworth \& Co., Boston, 1988).

Stewart, Kenneth R. "Ray Acoustical Model of the Ocean Using a Depth / Sound-Speed Profile with a Continuous First Derivative." J. Acoust. Soc. Am. 38, 339-347 (1965).

Van Calcar, Henry. "What You Need to Know to Improve LBS Submersible Tracking Performance." Proc. ROV'85, 43-51 (1985).

Von der Heydt, K. and E. Scheer, "CEAREX '89 Sensor Localization with the STS System," unpublished. 